October 30, 2018

hep-ph/0112004

\title{
Algebraic-Numerical Evaluation of Feynman Diagrams: Two-Loop Self-Energies
}

\author{
Giampiero Passarind and SAndro UcCirati \\ Dipartimento di Fisica Teorica, Università di Torino, Italy \\ INFN, Sezione di Torino, Italy
}

\begin{abstract}
A recently proposed scheme for numerical evaluation of Feynman diagrams is extended to cover all two-loop two-point functions with arbitrary internal and external masses. The adopted algorithm is a modification of the one proposed by F. V. Tkachov and it is based on the so-called generalized Bernstein functional relation. On-shell derivatives of self-energies are also considered and their infrared properties analyzed to prove that the method which is aimed to a numerical evaluation of massive diagrams can handle the infrared problem within the scheme of dimensional regularization. Particular care is devoted to study the general massive diagrams around their leading and non-leading Landau singularities.
\end{abstract}

PACS Classification: 11.10.-z; 11.15.Bt; 12.38.Bx; 02.90.+p

${ }^{*}$ Work supported by the European Union under contract HPRN-CT-2000-00149. 


\section{Introduction}

The evaluation of multi-loop Feynman diagrams has a long history [1] and, roughly speaking, we can say that there are theories more simple than others, noticeably QED (see for instance ref. [2]) and also to some extent QCD (see for instance ref. [4]), where we have few masses and the analytical approach can be pushed very far. Conversely the full electroweak Lagrangian shows several masses, ranging over a wide interval of values, therefore making the analytical evaluation of Feynman diagrams a complicated task. Equivalently complicated is the situation in any extension of the minimal standard model.

Soon or later the analytical approach will collapse and one can easily foresee that this failure will show up at the level of a complete two-loop calculation in the standard model or beyond. This calculation is, for instance, required to produce quantities as $\sin ^{2} \theta_{\text {eff }}^{l}$ [5] with a theoretical precision of $1 \times 10^{-6}$. For this reason one is lead to consider an alternative approach to the whole problem, namely to abandon the analytical way in favor of a fully automatic, numerical evaluation of multi-loop diagrams. The Holy Graal (General Recursive Applicative and Algorithmic Language) requires fast and accurate procedures to deal with the singularities of an arbitrary diagram.

For a fast and accurate numerical evaluation of arbitrary multi-loop Feynman diagrams there is some interesting proposal in the literature [6] that has been recently applied to the two-loop two-point sunset topology [7]. Older approaches can be found in ref. [8].

The Bernstein-Tkachov theorem [6] (hereafter BT) tells us that for any finite set of polynomials $V_{i}(x)$, where $x=\left(x_{1}, \ldots, x_{N}\right)$ is a vector of Feynman parameters, there exists an identity of the following form:

$$
\mathcal{P}(x, \partial) \prod_{i} V_{i}^{\mu_{i}+1}(x)=B \prod_{i} V_{i}^{\mu_{i}}(x) .
$$

where $\mathcal{P}$ is a polynomial of $x$ and $\partial_{i}=\partial / \partial x_{i} ; B$ and all coefficients of $\mathcal{P}$ are polynomials of $\mu_{i}$ and of the coefficients of $V_{i}(x)$. Any multi-loop Feynman diagram can be cast into the form of Eq.(1). Iterative applications of the BT functional relations, followed by integration by parts, allows us to express the integrand in parametric space as a combination of (polynomials) ${ }^{N} \times \operatorname{logarithms~of~}$ the same polynomials, therefore achieving a result that is well suited for numerical integration. The $B$ coefficients of Eq.(1) will contain all Landau singularities [9] of the corresponding diagram.

For general one-loop diagrams we have an explicit solution for the polynomial $\mathcal{P}$ which is due to F. V. Tkachov [6] (see also ref. [10] and ref. [11]). Any one-loop Feynman diagram $G$, irrespectively from the number of external legs, is expressible as

$$
G=\int_{S} d x V^{-\mu}(x)
$$

where the integration region is $x_{i} \geq 0, \sum_{i} x_{i} \leq 1$ and where $V(x)$ is a quadratic form of $x$,

$$
V(x)=x^{t} H x+2 K^{t} x+L .
$$

The solution to the problem of determining the polynomial $\mathcal{P}$ is as follows:

$$
\mathcal{P}=1-\frac{(x+X) \partial_{x}}{2(\mu+1)}, \quad X=K^{t} H^{-1}, \quad B=L-K^{t} H^{-1} K
$$


For $N$-loop diagrams with $N \geq 2$ a minimal BT approach has been recently proposed in ref. [7] where we have adopted a different strategy aimed to deal with arbitrary diagrams. It represents a compromise based on the simple observation that we know how to apply the BT-iterative procedure for arbitrary one-loop diagrams. Therefore, given any two-loop diagram $G$ we apply the BT functional relation to $G_{L}$, the one-loop sub-diagram of $G$ which has the largest number of internal lines. In this way the integrand for $G$ in $x$-space can be made smooth, a part from the factor $B$ of Eq.(1) which is now a polynomial in $x_{S}$, the Feynman parameters needed for the complementary sub-diagram of $G$ with the smallest number of internal lines, $G_{S}$. The sub-diagram $G_{S}$, after integration over its momentum, becomes an additional $-x_{S}$-dependent and with non-canonical power - propagator for $G_{L}$. This procedure can be immediately generalized to any number of loops. Furthermore, one should realize that the BT procedure, in short raising powers, does not introduce singularities through $B\left(x_{S}\right)$, a part from the singularities in the external parameters of the original diagram. Therefore, before performing the $x_{S}$-integration we move the integration contour into the complex hyper-plane, thus avoiding the crossing of apparent singularities, see also the work of ref. [12].

As we have anticipated, in this paper we extend the minimal BT-approach to cover all twoloop, two-points functions. In dealing with the sunset topology we have discovered a remarkable property that will be generalized in this paper to all two-loop two-point functions but that remains true for all two-loop three-point planar topologies. For all these diagrams, essentially, we do not have enough external momenta to make the matrix $H$ of Eq.(3) non-singular. As a consequence, a change of variables is always possible in the quadratic form $V$ that makes the $B$-coefficient of Eq.(1) independent on $x_{S}$, at least if we use only one iteration of the algorithm. Therefore, in these cases, no distortion of the integration path is needed. In this way we are able to be closer to the original idea of the BT-method. However, as already stressed in the original paper [6], this $B$ will vanish at some threshold. We are able to show that this occurs at some non-leading Landau singularity of the diagram where additional analytical work is needed before starting the numerical evaluation.

At the same time we have considered the problem of an efficient evaluation of infrared divergent multi-loop diagrams. Although the massless world is efficiently treated in QED and QCD within the analytical approach, any multi-loop calculation in the standard model or beyond is plagued by infrared divergences and we must contrive a scheme for dealing with them in a purely numerical way. For some of the two-loop two-point functions, the infrared divergent configurations are simple enough to allow for BT treatment with a consequent and straightforward analytical evaluation. The remaining topologies, in particular the one containing overlapping infrared configurations, require a novel approach.

Our solution is derived by adapting the general algorithm of ref. [17]: the residues of the infrared poles and the finite part of a multi-loop diagram can be cast into a form which allows for a reliable numerical integration. The algorithm is perfectly defined for all integrands that may vanish only at the boundaries of the parametric space. Here the strategy differs somehow from the BT approach. In the latter the main attempt is toward raising powers in the integrand while here the powers remain untouched and the whole idea is about factorizing the singular behavior 
into simple factors that can be integrated analytically, leaving non-singular terms to be treated numerically.

The outline of the paper will be as follows: in Section 2 we introduce the definitions and the basic properties for arbitrary two-loop diagrams. In Subsection 2.1 we discuss the so-called reducible two-loop diagrams while, in Section 1 we present special cases of the general BT functional relation. In Section 5 we start the evaluation of two-loop two-point functions with four internal lines. In Section 6 a general class of two-loop diagrams, those that contain a self-energy insertion, is discussed. Explicit methods for evaluating diagrams with four internal lines are given in Subsection 5.1 - 5.2, while their Landau singularities are computed in Subsection 5.3. Further refinements for diagrams with four internal lines are discussed in Subsection 5.4 - 5.8. The on-shell derivative of these diagrams and their infrared poles are analyzed in Subsection 5.9. In Subsection 5.10 the tensor integrals are studied. The two-loop two-point diagrams with five internal lines are presented and discussed in Section (7). In particular we discuss in Subsection 7.1 the simplest case in this class, in Subsection 7.2 its Landau singularities and in Subsection 7.3 its explicit evaluation. The corresponding on-shell derivative and its infrared poles are shown in Subsection 7.4. The most difficult topology with five internal lines is presented in Subsection 7.5, its Landau singularities in Subsection 7.6, its evaluation in Subsection 7.7 - 7.8. The corresponding on-shell derivative and its infrared poles are discussed in Subsection 7.9 while in Subsection 7.10 we analyze the tensor integrals of this class with a specific and realistic example shown in Subsection 7.11. Finally, numerical results and comparisons for those few cases where analytical work has been done are shown and discussed in Section 8. Technical details are discussed in various Appendices.

\section{Arbitrary two-loop diagrams}

In this section we present basic definitions and properties of diagrams. An arbitrary two-loop diagram has the following expression:

$$
\begin{aligned}
& \left(\alpha ; m_{1}, \ldots, m_{\alpha} ; \eta_{1}\left|\gamma ; m_{\alpha+1}, \ldots, m_{\alpha+\left(1-\gamma_{5}\right) m a} ; \eta_{12}\right| \beta ; m_{\alpha+\gamma+1}, \ldots, m_{\alpha+\gamma+\beta} ; \eta_{2}\right)= \\
& \frac{\mu^{2 \epsilon}}{\pi^{4}} \int d^{n} q_{1} d^{n} q_{2} \prod_{i=1}^{\alpha}\left(k_{i}^{2}+m_{i}^{2}\right)^{-1} \prod_{j=\alpha+1}^{\alpha+\gamma}\left(k_{j}^{2}+m_{j}^{2}\right)^{-1} \prod_{l=\alpha+\gamma+1}^{\alpha+\gamma+\beta}\left(k_{l}^{2}+m_{l}^{2}\right)^{-1},
\end{aligned}
$$

where $n=4-\epsilon$, with $n$ being the space-time dimension, and where $\alpha, \beta$ and $\gamma$ give the number of lines in the $q_{1}, q_{2}$ and $q_{1}-q_{2}$ loops respectively. Furthermore we have

$$
\begin{array}{rlrl}
k_{i} & =q_{1}+\sum_{j=1}^{N} \eta_{i j}^{1} p_{j}, & i & =1, \ldots, \alpha \\
k_{i}=q_{1}-q_{2}+\sum_{j=1}^{N} \eta_{i j}^{12} p_{j}, & i=\alpha+1, \ldots, \alpha+\gamma \\
k_{i}=q_{2}+\sum_{j=1}^{N} \eta_{i j}^{2} p_{j}, & i=\alpha+\gamma+1, \ldots, \alpha+\gamma+\beta,
\end{array}
$$

$N$ being the number of external legs, $\eta^{a}= \pm 1$, or 0 and $\{p\}$ the set of external momenta. Furthermore, $\mu$ is the arbitrary unit of mass. Diagrams that can always be reduced to combinations 
of other diagrams with less internal lines will never receive a particular name. Otherwise we will denote a two-loop diagram with

$$
G^{\alpha \beta \gamma}
$$

where $G=S, V, B$ etc. stands for two-, three-, four-point etc, and $\alpha, \beta$ and $\gamma$ give the number of lines in the $q_{1}, q_{2}$ and $q_{1}-q_{2}$ loops respectively. Family of diagrams with the same number $N$ of internal lines will be denoted collectively by $S_{N}$, etc.

Next we recall few basic properties of two-loop diagrams [18. In any two-loop diagram there are three one-loop sub-diagrams, called $\alpha \gamma, \beta \gamma$ and $\alpha \beta$ sub-diagrams respectively.

Definition 1: the $\alpha \beta \gamma$ diagram is overall ultraviolet (UV)convergent if $\alpha+\beta+\gamma>4$, logarithmically divergent if $\alpha+\beta+\gamma=4$, linearly etc. divergent if $\alpha+\beta+\gamma=3, \ldots$

Definition 2: the $\alpha \beta$ sub-diagram is convergent if $\alpha+\beta>2$, logarithmically divergent if $\alpha+\beta=2$, linearly etc. divergent if $\alpha+\beta=\frac{3}{2}, \ldots$

If any of the one-loop sub-diagrams diverge we have counter-terms associated with them. Therefore, in addition to the $\alpha \beta \gamma$ diagram we will also consider the subtraction diagrams of Fig. 1.

$$
G^{\underline{\alpha} \beta} \underline{\gamma}, \quad \text { etc., }
$$

The arbitrary $G^{\alpha \beta \gamma}$ diagram has the following representation in parametric space:

$$
\begin{aligned}
G^{\alpha \beta \gamma} & =-\left(\frac{\mu^{2}}{\pi}\right)^{\epsilon} \Gamma(\alpha+\beta+\gamma-4+\epsilon) \int d[x] \int d[y][X(1-X)]^{2-\alpha-\gamma-\epsilon / 2} y_{1}^{\alpha+\gamma-3+\epsilon / 2} \\
& \times\left\{M_{x}^{2} y_{1}+\sum_{l=\alpha+\gamma+1}^{\alpha+\beta+\gamma}\left(P_{2 l}^{2}+m_{l}^{2}\right) y_{l-\alpha-\gamma+1}-\left(P_{x} y_{1}+\sum_{l=\alpha+\gamma+1}^{\alpha+\beta+\gamma} P_{2 l} y_{l-\alpha-\gamma+1}\right)^{2}\right\}^{4-\alpha-\beta-\gamma-\epsilon},
\end{aligned}
$$

where the integration measures are

$$
d[x]=\int_{0}^{1} \prod_{i=1}^{\alpha+\gamma} d x_{i} \delta\left(\sum_{i=1}^{\alpha+\gamma} x_{i}-1\right), \quad d[y]=\int_{0}^{1} \prod_{i=1}^{\beta+1} d y_{i} \delta\left(\sum_{i=1}^{\beta+1} y_{i}-1\right),
$$

and parameter dependent masses and momenta are defined by

$$
\begin{aligned}
M_{x}^{2} & =\frac{R^{2}-K^{2}}{X(1-X)}, \quad P_{x}=\frac{P_{1}}{1-X}-\frac{P_{12}}{X} \\
K & =P_{1}+P_{12}, \quad P_{1}=\sum_{i=1}^{\alpha} x_{i} P_{1 i}, \quad P_{12}=\sum_{i=\alpha+1}^{\alpha+\gamma} x_{i} P_{12 i} \\
R^{2} & =\sum_{i=1}^{\alpha} x_{i}\left(P_{1 i}^{2}+m_{i}^{2}\right)+\sum_{i=\alpha+1}^{\alpha+\gamma} x_{i}\left(P_{12 i}^{2}+m_{i}^{2}\right) \\
P_{a i} & =\sum_{k=1}^{N} \eta_{i k}^{a} p_{k}, \quad X=\sum_{i=\alpha+1}^{\alpha+\gamma} x_{i}
\end{aligned}
$$



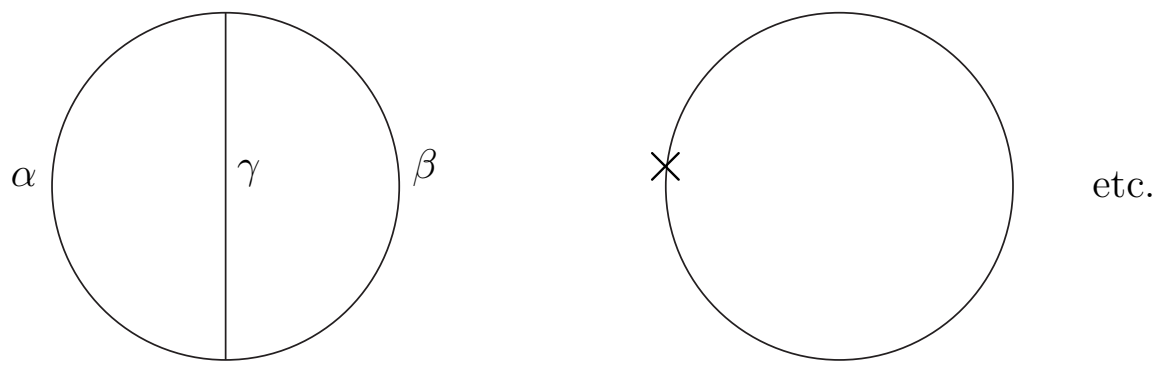

Figure 1: The arbitrary two-loop diagram $G_{L}^{\alpha \beta \gamma}$ of Eq.(5) and one of the associated subtraction subdiagrams.

\subsection{Reducible two-loop diagrams}

There are two-loop diagrams that can be easily reduced to products of one-loop diagrams, some of them occurring in the reduction of tensor integrals. A typical example is represented by

$$
A B\left(p^{2} ; m_{1}, m_{2}, m_{3}\right)=\frac{\mu^{2 \epsilon}}{\pi^{4}} \int \frac{d^{n} q_{1} d^{n} q_{2}}{\left(q_{1}^{2}+m_{1}^{2}\right)\left(q_{2}^{2}+m_{2}^{2}\right)\left(\left(q_{2}+p\right)^{2}+m_{3}^{2}\right)}=A_{0}\left(m_{1}\right) B_{0}\left(p^{2} ; m_{2}, m_{3}\right),
$$

where $A_{0}\left(B_{0}\right)$ is the one-loop, one-(two-)point scalar function [19]. This function satisfies

$$
A B\left(p^{2} ; m_{1}, m_{2}, m_{3}\right)=A B\left(p^{2} ; m_{1}, m_{3}, m_{2}\right)
$$

\section{Minimal BT algorithm}

For an arbitrary diagram the application of minimal BT algorithm requires distortion of the integration hyper-contour. First of all let us discuss how this can be done: we rely on the fact that applying BT functional relations to $G_{L}$ does not introduce new singularities. Consider any diagram: the integration hyper-contour can be distorted away from its original real location, a simplex described by $x_{i} \geq 0$ and $\sum x_{i} \leq 1$, and when the possibility of this distortion ceases, we encounter a singularity of the function [20]. The difficulty in locating the singularities lies in imagining what happens in the multi-dimensional (complex) space of integration. Consider now a simple example

$$
\begin{gathered}
G=\int_{0}^{1} d x \int_{0}^{x} d y V^{-1-\epsilon}(x, y)=\int_{0}^{1} d y f(y), \\
V(x, y)=H x^{2}+2 K(y) x+L(y) .
\end{gathered}
$$

Minimal BT algorithm transforms $G$ into

$$
\begin{aligned}
G & =\int_{0}^{1} d x \int_{0}^{x} d y \frac{1}{b(y)}\left[1+\frac{1}{2} \ln V(x, y)\right] \\
& -\frac{1}{2} \int_{0}^{1} d y \frac{1}{b(y)}\{[1+X(y)] \ln V(1, y)-[y+X(y)] \ln V(y, y)\}
\end{aligned}
$$


with $b=L-K^{2} / H$ and $X=K / H$. Let $y_{0}$ be a root of $b$, i.e. $b\left(y_{0}\right)=0$. What happens to $f\left(y_{0}\right)$ ? Since $b\left(y_{0}\right)=0$ we can use another identity

$$
\left[1+\frac{1}{2(1+\epsilon)}(x+X) \partial_{x}\right] V^{-1-\epsilon}\left(x, y_{0}\right)=0
$$

to derive

$$
f\left(y_{0}\right)=-\frac{1}{2(1+\epsilon)} \int_{y_{0}}^{1} d x(x+X) \partial_{x} V\left(x, y_{0}\right) .
$$

If there is a pinch singularity at $x=x_{0}$ with $y_{0}<x_{0}<1$ then $\left\{x_{0}, y_{0}\right\}$ is already a singularity of $G$. If not we integrate by parts and obtain

$$
(1+2 \epsilon) f\left(y_{0}\right)=\left(y_{0}+X\right) V^{-1-\epsilon}\left(y_{0}, y_{0}\right)-(1+X) V^{-1-\epsilon}\left(1, y_{0}\right)
$$

If $V\left(y_{0}, y_{0}\right)=0$ or $V\left(1, y_{0}\right)=0$ then $x=1, y=y_{0}$ or $x=y=y_{0}$ is an end-point singularity of $G$, if not then no new singularity is introduced since $f(y)$ is analytical in $y=y_{0}$.

To understand when minimal BT eventually fails we consider another simple example which, however, is general enough to complete our discussion. We start with a quadratic form

$$
\begin{gathered}
V(x, y)=H x^{2}+2 K(y) x+L(y), \\
H=m_{d}^{2}, \quad K=\frac{1}{2}\left(s-m_{u}^{2}-m_{d}^{2}\right) y-\frac{1}{2} M_{z}^{2}, \quad L=m_{u}^{2} y^{2}+\left(M_{w}^{2}-s\right) y+M_{z}^{2} .
\end{gathered}
$$

This is actually a $C_{0}$ function corresponding to a vertex correction for $W$ (off-shell) $\rightarrow \bar{u} d$. Applying minimal BT gives a $b(y)$ which is a quadratic form in $y$. The two roots $b\left(y_{ \pm}\right)=0$ are coincident when

$$
s=\frac{1}{2 m_{d}^{2}}\left[m_{u}^{2} M_{z}^{2}-M_{w}^{2} M_{z}^{2}+2 m_{d}^{2} M_{w}^{2}+m_{d}^{2} M_{z}^{2} \pm \lambda^{1 / 2}\left(M_{z}^{2}, m_{d}^{2}, m_{d}^{2}\right) \lambda^{1 / 2}\left(m_{u}^{2}, M_{w}^{2}, m_{d}^{2}\right)\right],
$$

which is the well-known anomalous threshold. Here $\lambda$ is the usual Källen-function [14]. Let $y_{L, R}$ the solutions of $V=0$, which are now branch points in Eq.(15). These roots are coincident for

$$
x=x_{\mathrm{th}}=\left(m_{u}^{2} M_{w}^{2}-2 m_{u}^{2} M_{z}^{2}-m_{u}^{2} s+m_{d}^{2} M_{w}^{2}-m_{d}^{2} s-M_{w}^{2} s+s^{2}\right) \lambda^{-1}\left(s, m_{u}^{2}, m_{d}^{2}\right),
$$

and one can easily verify that with $x$ and $s$ given by Eq.(22) and Eq.(21) respectively we have

$$
y_{ \pm}=y_{L}=y_{R}
$$

In this case, if $x_{\mathrm{th}} \in[0,1]$ and $y_{ \pm} \in[0,1]$ we cannot distort anymore the $y$ integration contour to avoid $y=y_{ \pm}$without crossing a cut of the logarithm. However we can still go on with our procedure if we distort the $x$ integration contour in order to avoid $x=x_{\mathrm{th}}$. This will be possible unless, for some specific configuration of the external parameters, we have $x_{\mathrm{th}}=0$ or $x_{\mathrm{th}}=1$. 
In our particular example this would require an unrealistic relation between $W, Z$ and up, down masses. Even in this case we still have a chance of continuing the derivation. Indeed we have used logarithms of quadratic forms which give a pair of branch points which can pinch the integration contour exactly where we would like to deform it. However, when only logarithms are present and not logarithms squared, we split the logarithm as

$$
\ln m_{u}^{2}+\eta\left(y-y_{L}, y-y_{R}\right)+\sum_{i=L, R} \ln \left(y-y_{i}\right)
$$

where $\eta$ is the usual Veltman's function, $\eta(a, b)=\ln (a b)-\ln a-\ln b$, and logarithms are defined with a cut along the negative real axis. The final expression for the diagram will be the sum of terms proportional to $\eta$-functions and terms of the form $\ln \left(y-y_{i}\right) / b(y)$. The former do not create a problem in distorting, when needed, the integration contour. For the latter, however, if one of the roots of $b$, or both, is real and internal to the interval $[0,1]$ then the distortion for the corresponding term must be examined with care. If the imaginary part of $y_{i}$ is positive(negative) then we move the contour into the negative(positive) imaginary half-plane, so that the cut of the logarithm will never be crossed. If $y_{i}$ is real and negative no problem will arise and we can distort in any of the two ways. If its is real and positive then the distortion is fixed according to the $i \delta$ prescription. The reason why we cannot apply this argument to cases where we have the presence of $\ln ^{2}$-terms is simple: after the splitting of the logarithm we will encounter terms proportional to $\ln \left(y-y_{L}\right) \ln \left(y-y_{R}\right)$. When the roots are complex conjugated we will have cuts both in the positive and in the negative imaginary half-planes and the possibility of distorting the contour ceases when they approach the real axis. Note that these quadratic terms are always present for ultraviolet divergent diagrams.

\section{Special BT relations}

In this section we present some explicit examples of the Bernstein-Tkachov functional relations. Let $V(x)$ be a quadratic form of a vector $x$,

$$
V(x)=x^{t} H x+2 K^{t} x+L,
$$

then the solution to the problem of determining the polynomial $\mathcal{P}$ is as given in Eq.(4). There are special BT functional relations: typically, when we have a quadratic in two variables $y, z$ of the form $V=h z^{2}+2 k_{1} z+2 k_{2} y+l$ then the matrix $H$ is singular. Here we use

$$
\left[1-\frac{y}{\mu+1} \partial_{y}-\frac{1}{2(\mu+1)}\left(z+\frac{k_{1}}{h}\right) \partial_{z}\right] V^{\mu+1}=\frac{h l-k_{1}^{2}}{h} V^{\mu} .
$$

For a quadratic in one variable, $V=h y^{2}+2 k y+l$ we use

$$
\left[1-\frac{1}{2(\mu+1)}\left(y+\frac{k}{h}\right) \frac{d}{d y}\right] V^{\mu+1}=\frac{h l-k^{2}}{h} V^{\mu}
$$


while for $V=k y+l$ we use

$$
\left[1-\frac{1}{\mu+1} y \frac{d}{d y}\right] V^{\mu+1}=l V^{\mu}, \quad\left[1+\frac{1}{\mu+1} \frac{1-V}{k} \frac{d}{d y}\right] V^{\mu+1}=V^{\mu} .
$$

\section{The $S_{4}$ family}

There are two diagrams in the $S_{4}$-family, both with $\alpha=\gamma=1$ and with $\beta=2$. The first is $S^{121}$ and is given in Fig. A. It is overall logarithmically divergent and the $\alpha \gamma$ sub-diagram is also logarithmically divergent. We have another diagram with the same number of internal lines, given in Fig. 3. This second diagram is given by the following integral:

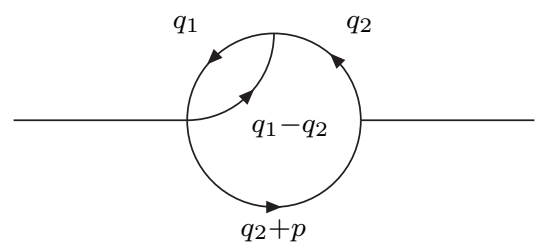

Figure 2: The two-loop diagram $S^{121}$ of Eq.(34). Arrows indicate the momentum flow.

$$
\left(1 ; m_{1}\left|1 ; m_{2}\right| 2, m_{3}, m_{4}\right)=\frac{\mu^{2 \epsilon}}{\pi^{4}} \int \frac{d^{n} q_{1} d^{n} q_{2}}{\left(q_{1}^{2}+m_{1}^{2}\right)\left(\left(q_{1}-q_{2}\right)^{2}+m_{2}^{2}\right)\left(q_{2}^{2}+m_{3}^{2}\right)\left(q_{2}^{2}+m_{4}^{2}\right)}
$$

Therefore, this case is trivially reduced to known diagrams. If $m_{3} \neq m_{4}$ then

$$
\begin{gathered}
\left(1 ; m_{1}\left|1 ; m_{2}\right| 2, m_{3}, m_{4}\right)=\frac{1}{m_{4}^{2}-m_{3}^{2}}\left[S^{111}\left(0 ; m_{1}, m_{2}, m_{3}\right)-S^{111}\left(0 ; m_{1}, m_{2}, m_{4}\right)\right], \\
\pi^{4} S^{111}=\mu^{2 \epsilon} \int \frac{d^{n} q_{1} d^{n} q_{2}}{\left(q_{1}^{2}+m_{1}^{2}\right)\left(\left(q_{1}-q_{2}+p\right)^{2}+m_{2}^{2}\right)\left(q_{2}^{2}+m_{3}^{2}\right)} .
\end{gathered}
$$

otherwise we will have

$$
\left(1 ; m_{1}\left|1 ; m_{2}\right| 2, m_{3}, m_{4}\right)=(\epsilon-1) S_{33}\left(0 ; m_{1}, m_{2}, m_{3}=m_{4}\right),
$$

where $S_{33}$ has been introduced in ref. [0] as the following integral:

$$
\pi^{4} S_{33}=\frac{\mu^{2 \epsilon}}{\epsilon-1} \int \frac{d^{n} q_{1} d^{n} q_{2}}{\left(q_{1}^{2}+m_{1}^{2}\right)\left(\left(q_{1}-q_{2}+p\right)^{2}+m_{2}^{2}\right)\left(q_{2}^{2}+m_{3}^{2}\right)^{2}}
$$




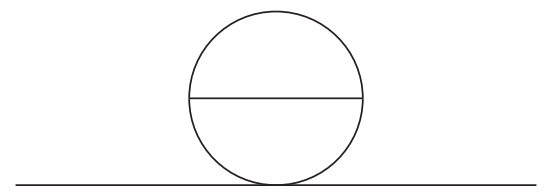

Figure 3: The second diagram of the $S_{4}$-family, Eq.(30), which is evaluated in terms of $S^{111}$ (ref. [0]) or $S_{33}$ diagrams (Eq.(33)) at zero external momentum.

The $S^{121}$ topology is given in terms of the following integral:

$$
\pi^{4} S^{121}=\mu^{2 \epsilon} \int \frac{d^{n} q_{1} d^{n} q_{2}}{\left(q_{1}^{2}+m_{1}^{2}\right)\left(\left(q_{1}-q_{2}\right)^{2}+m_{2}^{2}\right)\left(q_{2}^{2}+m_{3}^{2}\right)\left(\left(q_{2}+p\right)^{2}+m_{4}^{2}\right)} .
$$

One way to evaluate $S^{121}$ starts with the introduction of a Feynman parameter $x$ for the $q_{1^{-}}$ loop; successively, we integrate over $q_{1}$ and, finally, combine the $q_{2}$-dependent propagators with parameters $y, z$. After performing the $q_{2}$-integration we obtain

$$
\begin{aligned}
S^{121} & =-2\left(\frac{\mu^{2}}{\pi s}\right) \Gamma(\epsilon) \int_{0}^{1} d x \int_{0}^{1} d y \int_{0}^{y} d z[x(1-x)]^{-\epsilon / 2}(1-y)^{\epsilon / 2} \\
& \times\left[\left(\mu_{x}^{2}-z^{2}\right) \chi_{121}^{-1-\epsilon}+\frac{2-\epsilon / 2}{\epsilon} \chi_{121}^{-\epsilon}\right],
\end{aligned}
$$

where the quadratic form $\chi_{121}(x, y, z)$ is defined by

$$
\chi_{121}(x, y, z)=z^{2}-\mu_{34}^{2} z+\left(\mu_{3}^{2}-\mu_{x}^{2}\right) y+\mu_{x}^{2},
$$

and where we have introduced additional auxiliary quantities,

$$
\mu_{x}^{2}=\frac{(1-x) \mu_{1}^{2}+x \mu_{2}^{2}}{x(1-x)}=\frac{\mu_{12}^{2}}{x(1-x)}, \quad \mu_{34}^{2}=1+\mu_{3}^{2}-\mu_{4}^{2} .
$$

In Eq. (37) we have assumed that $p^{2}=-s$ and $s>0$. Scaled masses are, therefore, defined to be $m^{2}=\mu^{2} s$. If, instead, $p^{2}$ is positive, we will have to change the procedure as described in the next subsection.

\subsubsection{The case $p^{2}=-t$ with $t<0$}

Starting from the quadratic form

$$
U=(y-z) m_{3}^{2}+z m_{4}^{2}+(1-y) m_{x}^{2}+z(1-z) p^{2},
$$

with $p^{2}=-t$ and $t$ negative we redefine scaled masses to be $m^{2}=-\mu^{2} t$ and obtain $U=-t \chi$,

$$
\chi=-z^{2}+\mu_{43}^{2} z+\left(\mu_{3}^{2}-\mu_{x}^{2}\right) y+\mu_{x}^{2}, \quad \text { with } \quad \mu_{43}^{2}=1+\mu_{4}^{2}-\mu_{3}^{2} .
$$

The signs in this quadratic form will have an effect on the raising procedure, as described below in Subsection 5.1. 


\subsection{Evaluation of $S^{121}$ : method I}

In the following sections we will be able to present several derivations for $S^{121}$ and to compare the corresponding numerical results. In order to evaluate $S^{121}$ we may use the following relation:

$$
\chi_{121}^{-1-\epsilon}(x, y, z)=-\frac{4}{b_{121}}\left[1+\frac{1}{\epsilon}\left(P_{y} \partial_{y}+P_{z} \partial_{z}\right)\right] \chi_{121}^{-\epsilon}(x, y, z),
$$

with polynomials $P_{y, z}$ given by

$$
P_{y}=y, \quad P_{z}=\frac{1}{2}\left(z-\frac{1}{2} \mu_{34}^{2}\right), \quad b_{121}=\mu_{34}^{4}-4 \mu_{x}^{2} .
$$

If $p^{2}=-t$ and $t$ is negative, the definition of $b_{121}$ will change accordingly,

$$
b_{121} \rightarrow \mu_{43}^{4}+4 \mu_{x}^{2}>0
$$

so that $b_{121}$ is never zero in the $t$-channel, as expected.

We will also use an additional quadratic form, $X_{121}$, which is related to $\chi_{121}$ (Eq.(37)), by

$$
\begin{aligned}
X_{121}(x, y, z) & =x(1-x) \chi_{121}(x, y, z) \\
& =x(1-x)\left[-z(1-z)+\mu_{4}^{2} z+\mu_{3}^{2}(y-z)\right]+\left[(1-x) \mu_{1}^{2}+x \mu_{2}^{2}\right](1-y),
\end{aligned}
$$

The next ingredient needed in the evaluation of the diagram is integration by parts which is performed with the help of the following relations:

$$
\begin{gathered}
\int_{0}^{y} d z z^{n} \partial_{z} \ln ^{m} X_{121}(x, y, z)=y^{n} \ln ^{m} X_{121}(x, y, y)-\delta_{0, n} \ln ^{m} X_{121}(x, y, 0) \\
-n \int_{0}^{y} d z z^{n-1} \ln ^{m} X_{121}(x, y, z) \\
\int_{z}^{1} d y y^{n}(1-y)^{\epsilon / 2} \partial_{y} \ln ^{m} X_{121}(x, y, z)=-z^{n}(1-z)^{\epsilon / 2} \ln ^{m}\left(X_{121}(x, z, z)\right) \\
-\int_{z}^{1} d y\left[n y^{n-1}(1-y)^{\epsilon / 2}-\frac{\epsilon}{2} y^{n}(1-y)^{\epsilon / 2-1}\right] \ln ^{m} X_{121}(x, y, z) .
\end{gathered}
$$

Furthermore, we will use

$$
\begin{aligned}
& \int_{z}^{1} d y y^{n}(1-y)^{\epsilon / 2-1} \ln ^{m} X_{121}(x, y, z)=\frac{2}{\epsilon}(1-z)^{\epsilon / 2} \ln ^{m} X_{121}(x, 1, z) \\
+ & \int_{z}^{1} d y(1-y)^{\epsilon / 2-1}\left[y^{n} \ln ^{m} X_{121}(x, y, z)-\ln ^{m} X_{121}(x, 1, z)\right] .
\end{aligned}
$$

Before discussing the finite part of $S^{121}$ we consider its ultraviolet poles. As usual, the pole parts can be computed analytically. The double pole of $S^{121}$ is easily derived and the corresponding expression, where in the spirit of the $\overline{M S}$ scheme we include a bunch of constants, reads as follows:

$$
S_{\text {double-pole }}^{121}=-\frac{2}{\epsilon^{2}}-\Delta_{U V}^{2}
$$


where, for the constants, we obtain

$$
\Delta_{U V}=\gamma+\ln \pi+\ln \frac{s}{\mu^{2}}
$$

Here, $\gamma=0.577216$ is the Euler's constant and $\mu$ is the arbitrary unit of mass. The single-pole requires some extra work to be discussed in Subsection 5.2 .

\subsection{Single-pole and finite part of $S^{121}$}

For computing the single ultraviolet pole of $S^{121}$ we use the relation

$$
\frac{\mu_{x}^{2}-z^{2}}{\chi_{121}}=1-\left(z \partial_{z}+y \partial_{y}\right) \ln \chi_{121},
$$

and derive the following result:

$$
S_{\text {single-pole }}^{121}=\frac{R_{4}}{\epsilon}+\text { constants, }
$$

where we also include a bunch of constant into the definition and where

$$
R_{4}=2\left(\gamma+\ln \pi+\ln \frac{s}{\mu^{2}}\right)-1+2 \int_{0}^{1} d x \int_{0}^{1} d y \ln \chi_{121}(x, 1, y) .
$$

Since $\chi_{121}(x, 1, y)=y^{2}-\mu_{34}^{2} y+\mu_{3}^{2}$, we find

$$
R_{4}=-2 B_{0}^{\mathrm{fin}}\left(-s ; m_{3}, m_{4}\right)
$$

which is the finite part of the one-loop two-point function corresponding to internal masses $m_{3}$ and $m_{4}$. This is exactly the result expected, from general arguments, to compensate the overlapping divergency coming from the associated subtraction diagram of Fig. 4 . This result proves cancellation of poles with logarithmic residues, leaving only harmless poles, i.e. those with as

residue a polynomial of finite order in the external momenta. Summarizing our findings for the

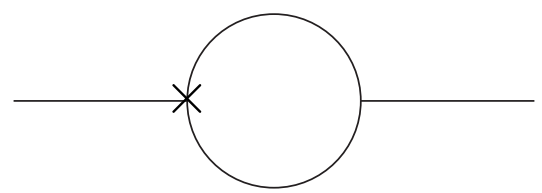

Figure 4: The subtraction diagram, containing a one-loop counter-term (represented by a $\times$ ) associated with the two-loop diagram $S^{121}$ of Fig. 2. 
ultraviolet divergent part of $S^{121}$, we obtain

$$
S_{\text {single-pole }}^{121}=\frac{1}{\epsilon}\left(2 \Delta_{U V}-1\right)+\Delta_{U V}+2\left(\frac{1}{\epsilon}-\Delta_{U V}\right) \int_{0}^{1} d y \ln \chi_{121}(x, 1, y),
$$

where one should note that $\chi_{121}(x, 1, y)$ is $x$-independent.

For the finite part of $S^{121}$ we have to consider the following quadratic forms that arise after integration by parts:

$$
\begin{aligned}
& \chi_{121}(x, y, z)=(y-z) \mu_{3}^{2}+z \mu_{4}^{2}+(1-y) \mu_{x}^{2}-z(1-z), \\
& \chi_{121}(x, y, y)=y \mu_{4}^{2}+(1-y) \mu_{x}^{2}-y(1-y) \\
& \chi_{121}(x, y, 0)=y \mu_{3}^{2}+(1-y) \mu_{x}^{2} \\
& \chi_{121}(x, 1, y)=(1-y) \mu_{3}^{2}+y \mu_{4}^{2}-y(1-y) .
\end{aligned}
$$

Before continuing the evaluation of the finite part of $S^{121}$, however, we need to understand its analytical structure. The tools for extracting all the singularities of the diagrams are, as usual, represented by the corresponding set of Landau equations.

\subsection{Landau equations for $S^{121}$}

Before starting the evaluation of this topology it is important to know as much as possible about its singularities as a function of $p^{2}$ and of the internal masses. The corresponding Landau equations are given by

$$
\begin{array}{ll}
\alpha_{1}\left(q_{1}^{2}+m_{1}^{2}\right)=0 & \alpha_{2}\left(\left(q_{1}-q_{2}\right)^{2}+m_{2}^{2}\right)=0 \\
\alpha_{3}\left(q_{2}^{2}+m_{3}^{2}\right)=0 & \alpha_{4}\left(\left(q_{2}^{2}+p\right)^{2}+m_{4}^{2}\right)=0
\end{array}
$$

and also by

$$
\alpha_{1} q_{1 \mu}+\alpha_{2}\left(q_{1}-q_{2}\right)_{\mu}=0, \quad-\alpha_{2}\left(q_{1}-q_{2}\right)_{\mu}+\alpha_{3} q_{2 \mu}+\alpha_{4}\left(q_{2}+p\right)_{\mu}=0
$$

The leading Landau singularity occurs for $\alpha_{i} \neq 0, \forall i$. We multiply the two equations Eq. (55) by $q_{1 \mu}, q_{2 \mu}$ and $p_{\mu}$ respectively. This gives an homogeneous system of six equations. If all $\alpha_{i}$ are different from zero, the singularity will occur for

$$
\begin{array}{ll}
q_{1}^{2}=-m_{1}^{2} & q_{2}^{2}=-m_{3}^{2}, \\
q_{1} \cdot q_{2}=\frac{1}{2}\left(m_{2}^{2}-m_{1}^{2}-m_{3}^{2}\right) & p \cdot q_{2}=\frac{1}{2}\left(s+m_{3}^{2}-m_{4}^{2}\right) .
\end{array}
$$

The Landau equations become as follows:

$$
\begin{aligned}
& -2 m_{1}^{2} \alpha_{1}+\left(m_{3}^{2}-m_{1}^{2}-m_{2}^{2}\right) \alpha_{2}=0, \\
& -\left(m_{3}^{2}+m_{1}^{2}-m_{2}^{2}\right) \alpha_{1}+\left(m_{3}^{2}-m_{1}^{2}+m_{2}^{2}\right) \alpha_{2}=0, \\
& p \cdot q_{1} \alpha_{1}+\left[p \cdot q_{1}+\frac{1}{2}\left(m_{4}^{2}-m_{3}^{2}-s\right)\right] \alpha_{2}=0, \\
& \left(m_{1}^{2}+m_{2}^{2}-m_{3}^{2}\right) \alpha_{2}+\left(m_{2}^{2}-m_{1}^{2}-m_{3}^{2}\right) \alpha_{3}+\left[2 p \cdot q_{1}+m_{2}^{2}-m_{1}^{2}-m_{3}^{2}\right] \alpha_{4}=0, \\
& \left(m_{1}^{2}-m_{2}^{2}-m_{3}^{2}\right) \alpha_{2}-2 m_{3}^{2} \alpha_{3}+\left(s-m_{3}^{2}-m_{4}^{2}\right) \alpha_{4}=0, \\
& {\left[s+m_{3}^{2}-m_{4}^{2}-2 p \cdot q_{1}\right] \alpha_{2}+\left(s+m_{3}^{2}-m_{4}^{2}\right) \alpha_{3}-\left(s-m_{3}^{2}+m_{4}^{2}\right) \alpha_{4}=0 .}
\end{aligned}
$$


There are two compatibility conditions for the first three equations that can be used to derive

$$
p \cdot q_{1}=\frac{m_{1}}{2\left(m_{1}+m_{2}\right)}\left[s+\left(m_{1}+m_{2}\right)^{2}-m_{4}^{2}\right] .
$$

Furthermore we must have a relation among masses, i.e.

$$
m_{3}^{2}=\left(m_{1}+m_{2}\right)^{2} .
$$

From these considerations we derive that

$$
\alpha_{1}=\frac{m_{2}}{m_{1}} \alpha_{2}, \quad \alpha_{2}=\frac{1}{m_{2}\left(m_{1}+m_{2}\right)}\left\{-\left(m_{1}+m_{2}\right)^{2} \alpha_{3}+\frac{1}{2}\left[s-\left(m_{1}+m_{2}\right)^{2}-m_{4}^{2}\right] \alpha_{4}\right\},
$$

is a solution for the system if the following condition is satisfied:

$$
s=\left(m_{1}+m_{2} \pm m_{4}\right)^{2} .
$$

Note that we have obtained the leading Landau singularity, i.e. all $\alpha_{i} \neq 0$, as

$$
s=\left(m_{1}+m_{2} \pm m_{4}\right)^{2}, \quad m_{3}^{2}=\left(m_{1}+m_{2}\right)^{2} .
$$

Naturally we have that

$$
s=\left(m_{1}+m_{2} \pm m_{4}\right)^{2}, \quad \alpha_{3}=0,
$$

are sub-leading Landau singularities corresponding to the normal and pseudo thresholds of the reduced diagram where the line corresponding to the propagator $q_{2}^{2}+m_{3}^{2}$ is contracted to a point. Similarly,

$$
s=\left(m_{3} \pm m_{4}\right)^{2}, \quad \alpha_{1}=\alpha_{2}=0,
$$

are the sub-leading ones corresponding to the reduced diagram where the $q_{1}$ sub-diagram is shrunk to a point.

\subsection{One iteration for $S^{121}$}

Having discussed the analytical properties of the diagram we continue the derivation of an integral representation which is particularly suited for numerical treatment. After one iteration of the raising procedure the result for $S^{121}$ is still very compact. First we introduce a combination of logarithms,

$$
L_{ \pm}(x, y, z)=\mp \ln X(x, y, z) \pm[\ln (1-y)+\ln x+\ln (1-x)]
$$

and obtain for the finite part of $S^{121}$

$$
\begin{aligned}
S_{\mathrm{fin}}^{121} & =\frac{1}{B_{121}} \int_{0}^{1} d x\left[\int_{0}^{1} d y \int_{0}^{y} d z I_{3}^{121}+\int_{0}^{1} d y I_{2}^{121}+I_{1}^{121}\right] \\
& +2 \int_{0}^{1} d x \int_{0}^{1} d y \int_{0}^{y} d z \ln X_{121}(x, y, z)\left[L_{+}(x, y, z)-\frac{1}{2}\right]-\frac{21}{4}-\frac{1}{2} \zeta(2) .
\end{aligned}
$$


where $B_{121}$ follows from Eq.(41) and becomes

$$
B_{121}=x(1-x) b_{121}=x(1-x) \mu_{34}^{4}-4 \mu_{12}^{2},
$$

with $\mu_{12}^{2}$ and $\mu_{34}^{2}$ defined in Eq.(37). and the various integrands appearing inside Eq.(65) are as follows:

$$
\begin{aligned}
& I_{3}^{121}=-\ln X_{121}(x, y, z)\left\{4\left(\mu_{12}^{2}-X z^{2}\right)-2 L_{+}(x, y, z)\left[3 \mu_{12}^{2}-X z\left(5 z-\mu_{34}^{2}\right)\right]\right\} \\
& -4\left(X z^{2}-\mu_{12}^{2}\right)\left(\frac{\ln X_{121}(x, y, z)}{y-1}\right)_{+} \\
& I_{2}^{121}=-4\left(X y^{2}-\mu_{12}^{2}\right) \ln X_{121}(x, 1, y) L_{-}(x, 1, y)-\left(\mu_{34}^{2}+2 y\right)\left(X y^{2}-\mu_{12}^{2}\right) \\
& \times \ln X_{121}(x, y, y) L_{+}(x, y, y)-\mu_{12}^{2} \mu_{34}^{2} \ln X_{121}(x, y, 0) L_{+}(x, y, 0), \\
& I_{1}^{121}=-\ln X\left(\frac{1}{3} X-2 \mu_{12}^{2}\right)-3 \mu_{12}^{2}+\frac{25}{36} X .
\end{aligned}
$$

Here $\zeta(z)$ is the Riemann zeta-function [16], $X=x(1-x)$ and two additional quantities have been introduced:

$$
\mu_{12}^{2}=(1-x) \mu_{1}^{2}+x \mu_{2}^{2}, \quad \mu_{34}^{2}=1+\mu_{3}^{2}-\mu_{4}^{2} .
$$

The '+'-distribution is defined, as usual, by its action on a generic test function $f(x)$ :

$$
\int_{0}^{1} d x\left(\frac{f(x)}{x-1}\right)_{+}=\int_{0}^{1} d x \frac{f(x)-f(1)}{x-1} .
$$

If we stop the chain of iterations at this level we will have the advantage of a result that contains only one denominator, namely $b_{121}$. A second iteration, described in the Section 5.7, makes the total integrand smoother at the price of introducing additional denominators. If $p^{2}=-t$ with $t$ negative we define scaled masses as $m^{2}=-\mu^{2} t$ and derive

$$
\begin{aligned}
I_{3}^{121} & =\left\{-4\left(X z^{2}+\mu_{12}^{2}\right)+2\left[X\left(5 z^{2}-\mu_{43}^{2} z\right)+3 \mu_{12}^{2}\right] L_{+}(x, y, z)\right\} \ln X_{121}(x, y, z) \\
& +4\left(X z^{2}+\mu_{12}^{2}\right)\left(\frac{\ln X_{121}(x, y, z)}{y-1}\right)_{+}, \\
I_{2}^{121} & =\left(X y^{2}+\mu_{12}^{2}\right)\left(\mu_{43}^{2}+2 y\right) \ln X_{121}(x, y, y) L_{+}(x, y, y)-\mu_{43}^{2} \mu_{12}^{2} \ln X_{121}(x, y, 0) L_{+}(x, y, 0) \\
& +4\left(X y^{2}+\mu_{12}^{2}\right) \ln X_{121}(x, 1, y) L_{-}(x, 1, y) \\
I_{1}^{121} & =\left(\frac{1}{3} X+2 \mu_{12}^{2}\right) \ln X-\frac{25}{36} X-3 \mu_{12}^{2},
\end{aligned}
$$

instead of Eqs.(67)-(69). As usual, the iterative procedure of raising powers in the integrand produces apparent singularities that make the final result unstable in known regions of the $x$ integration. In the next subsection we will explain how to cure these instabilities. 


\subsection{Distorting the integration contour for $S^{121}$}

Obviously, there are numerical instabilities when $x_{ \pm}^{0}$, the zeros of $b_{121}$ (Eq.(41)), are real and internal to the integration domain. This can be avoided by distorting the $x$ integration contour in Eq.(65) into the complex plane. However, this possibility ceases when $x_{L, R}^{0}$, the roots of the quadratic form $X_{121}(x, y, z)$ (Eq.433)) pinch the real $x$-axis. Given that $X_{121}(x, y, z)=a x^{2}+b x+c$, this situation will occur when we simultaneously have

$$
x_{-}^{0}=x_{+}^{0}=-\frac{b}{2 a} \quad \text { and } \quad b^{2}=4 a c .
$$

The condition for coincidence, i.e. $x_{-}^{0}=x_{+}^{0} \in[0,1]$, is

$$
\mu_{34}^{2}=2\left(\mu_{1}+\mu_{2}\right)
$$

The remaining two conditions, namely $\operatorname{Im} x_{L, R}^{0}=0$ and $x_{L}^{0}=x_{R}^{0}=x_{-}^{0}=x_{+}^{0}$, require

$$
\left(\mu_{3}^{2}-\mu_{+}^{2}\right) y+\left(z-\mu_{+}\right)^{2}=0,
$$

where $\mu_{+}=\mu_{1}+\mu_{2}$. There are, therefore, two solutions for $z$, given by

$$
z=\mu_{+} \pm\left[\left(\mu_{+}^{2}-\mu_{3}^{2}\right) y\right]^{1 / 2}
$$

and, as long as we can distort the $z$ integration contour to avoid these points then the $x$ contour will not be pinched. This is always possible unless a new pinch will occur, which indeed is the case if $\mu_{3}=\mu_{+}$. Note that $y=0$ and $z=\mu_{+}$is outside the physical region where $0 \leq z \leq y$. By inserting $\mu_{3}=\mu_{+}$inside the relation $\mu_{34}^{2}=2\left(\mu_{1}+\mu_{2}\right)$ we obtain $\mu_{+} \pm \mu_{4}=1$ and the two correspond to the leading Landau singularity.

There are also logarithms of quadratic forms in two variables, $x$ and $y$. For $X_{121}(x, y, y)$ the $x$-contour is pinched for

$$
y=\mu_{+}+\frac{1}{2}\left\{\mu_{+}^{2}-\mu_{3}^{2} \pm\left[\left(\mu_{3}^{2}-\mu_{+}^{2}\right)\left(\mu_{3}^{2}-\mu_{+}^{2}-4 \mu_{+}\right)\right]^{1 / 2}\right\} .
$$

Therefore, the $y$ contour will be pinched if $\mu_{3}^{2}=\mu_{+}^{2}$ or $\mu_{3}^{2}=\mu_{+}^{2}+4 \mu_{+}$. Only the former is physical, corresponding to $y=\mu_{+}$, while the latter corresponds to $y=-\mu_{+}$.

For $X_{121}(x, y, 0)$ the $x$-pinching occurs for $y=-\mu_{+}^{2} /\left(\mu_{3}^{2}-\mu_{+}^{2}\right)$ so that it is enough to distort the $y$-integration contour avoiding this point. Finally, for $X_{121}(x, 1, y)$ the solution is

$$
y=\mu_{+} \pm\left(\mu_{+}^{2}-\mu_{3}^{2}\right)^{1 / 2}
$$

showing, again a pinch for $\mu_{3}=\mu_{+}$. Around these points we must introduce an alternative derivation. 


\subsection{Around the leading Landau singularity: method II}

When we are in the regions of external parameters that correspond to the leading Landau singularity of $S^{121}$, an alternative approach must be used. We easily derive the following relation:

$$
S^{121}=(\epsilon-1) \int_{0}^{1} d x S_{33}\left(x^{2} p^{2} ; m_{1}, m_{2}, M_{x}\right), \quad \text { with } \quad M_{x}^{2}=-p^{2} x^{2}+\left(p^{2}+m_{4}^{2}-m_{3}^{2}\right) x+m_{3}^{2},
$$

where $S_{33}$ (Eq.(33)) has been introduced in ref. [7]. The finite part of $S_{33}$ is

$$
\begin{aligned}
(\epsilon-1) S_{33}^{\operatorname{fin}}\left(x^{2} p^{2} ; m_{1}, m_{2}, M_{x}\right) & =-\int_{0}^{1} d y \int_{0}^{1} d z\left[\ln \xi(y, z)+\left(\frac{\ln \xi(y, z)}{z-1}\right)_{+}\right] \\
& +\ln \frac{M_{x}^{2}}{s}\left(2-\ln \frac{M_{x}^{2}}{s}\right)-\frac{7}{2}-\frac{1}{2} \zeta(2),
\end{aligned}
$$

and $\xi$ is given by

$$
\xi\left(y, z ; \mu_{1}, \mu_{2}, \mu_{3}\right)=z y(y-z)\left[(1-z) x^{2}-\mu_{3}^{2}\right]+(1-z)\left[\left(\mu_{2}^{2}-\mu_{1}^{2}\right) y+\mu_{1}^{2}\right],
$$

and must be evaluated at $\mu_{3}^{2}=M_{x}^{2} / s$.

\subsection{Two iterations for $S^{121}$}

In this section we return to the method described in Section 5.1; an additional iteration of the procedure requires the consideration of new quadratic forms in two or one variables. In particular, when we apply integration by parts, some care is needed in dealing with terms containing $\ln (1-y)$. For instance, we will use

$$
\begin{aligned}
\int_{0}^{1} d y \int_{0}^{y} d z \ln \chi_{121}(x, y, z) \ln (1-y) & =\int_{0}^{1} d z \int_{z}^{1} d y \ln \chi_{121}(x, y, z) \ln (1-y) \\
\int_{0}^{1} d z \int_{z}^{1} d y y^{n} \ln \chi_{121}(x, y, z) \ln (1-y) & =\int_{0}^{1} d z \int_{z}^{1} d y y^{n} \ln \frac{\chi_{121}(x, y, z)}{\chi_{121}(x, 1, y)} \ln (1-y) \\
& +\int_{0}^{1} d z \ln \chi_{121}(x, 1, z) \int_{0}^{1-z} d y(1-y)^{n} \ln y
\end{aligned}
$$

With only two variables we use

$$
\begin{aligned}
\int_{0}^{1} d y y^{n} \ln \chi_{121}(x, y, y) \ln (1-y) & =\int_{0}^{1} d y y^{n} \ln \frac{\chi_{121}(x, y, y)}{\chi_{121}(x, 1,1)} \ln (1-y) \\
& -\frac{1}{n+1} \ln \chi_{121}(x, 1,1) \sum_{j=1}^{n+1} \frac{1}{j} \\
\int_{0}^{1} d y y^{n} \ln \chi_{121}(x, y, 0) \ln (1-y) & =\int_{0}^{1} d y y^{n} \ln \frac{\chi_{121}(x, y, 0)}{\chi_{121}(x, 1,0)} \ln (1-y) \\
& -\frac{1}{n+1} \ln \chi_{121}(x, 1,0) \sum_{j=1}^{n+1} \frac{1}{j}
\end{aligned}
$$


The second iteration of the rasing procedure is based on the following set of identities:

$$
\begin{aligned}
\ln \chi_{121}(x, y, y) & =-\frac{4}{b_{1 ; 121}}\left\{\frac{1}{2}\left(y+Y_{1}\right) \partial_{y} \chi_{121}(x, y, y)\left[1-\ln \chi_{121}(x, y, y)\right]\right. \\
& \left.+\chi_{121}(x, y, y) \ln \chi_{121}(x, y, y)\right\}, \\
\ln ^{2} \chi_{121}(x, y, y) & =\frac{8}{b_{1 ; 121}}\left\{\frac { 1 } { 2 } ( y + Y _ { 1 } ) \partial _ { y } \left[\chi_{121}(x, y, y)-\chi_{121}(x, y, y) \ln \chi_{121}(x, y, y)\right.\right. \\
& \left.\left.+\frac{1}{2} \chi_{121}(x, y, y) \ln ^{2} \chi_{121}(x, y, y)\right]-\frac{1}{2} \chi_{121}(x, y, y) \ln ^{2} \chi_{121}(x, y, y)\right\} .
\end{aligned}
$$

Furthermore, we will use an additional relation:

$$
\begin{aligned}
\int_{0}^{1} d y y^{n} \partial_{y} \chi_{121}(x, y, y) \ln ^{m} \chi_{121}(x, y, y) & =\chi_{121}(x, 1,1) \ln ^{m} \chi_{121}(x, 1,1) \\
& -\delta_{n, 0} \chi_{121}(x, 0,0) \ln ^{m} \chi_{121}(x, 0,0) \\
& -n \int_{0}^{1} d y y^{n-1} \chi_{121}(x, y, y) \ln ^{m} \chi_{121}(x, y, y) .
\end{aligned}
$$

Here we have introduced the following quantities:

$$
Y_{1}=-\frac{1}{2}\left(1-\mu_{4}^{2}+\mu_{x}^{2}\right), \quad b_{1 ; 121}=\lambda\left(1, \mu_{4}^{2}, \mu_{x}^{2}\right),
$$

with special cases given by $\chi_{121}(x, 1,1)=\mu_{4}^{2}$ and $\chi_{121}(x, 0,0)=\mu_{x}^{2}$. Similarly, for $\chi_{121}(x, 1, y)$, we obtain

$$
\begin{aligned}
\ln \chi_{121}(x, 1, y) & =-\frac{4}{b_{2 ; 121}}\left\{\frac{1}{2}\left(y+Y_{2}\right) \partial_{y} \chi_{121}(x, 1, y)\right. \\
& \left.\times\left[1-\ln \chi_{121}(x, 1, y)\right]+\chi_{121}(x, 1, y) \ln \chi_{121}(x, 1, y)\right\}, \\
\ln ^{2} \chi_{121}(x, 1, y) & =\frac{8}{b_{2 ; 121}}\left\{\frac { 1 } { 2 } ( y + Y _ { 2 } ) \partial _ { y } \left[\chi_{121}(x, 1, y)-\chi_{121}(x, 1, y) \ln \chi_{121}(x, 1, y)\right.\right. \\
& \left.\left.+\frac{1}{2} \chi_{121}(x, 1, y) \ln ^{2} \chi_{121}(x, 1, y)\right]-\frac{1}{2} \chi_{121}(x, 1, y) \ln ^{2} \chi_{121}(x, 1, y)\right\} .
\end{aligned}
$$

Furthermore, the following identities hold:

$$
\begin{aligned}
\int_{0}^{1} d y y^{n} \partial_{y} \chi_{121}(x, 1, y) \ln ^{m} \chi_{121}(x, 1, y) & =\chi_{121}(x, 1,1) \ln ^{m} \chi_{121}(x, 1,1) \\
& -\delta_{n, 0} \chi_{121}(x, 1,0) \ln ^{m} \chi_{121}(x, 1,0) \\
& -n \int_{0}^{1} d y y^{n-1} \chi_{121}(x, 1, y) \ln ^{m} \chi_{121}(x, 1, y), \\
\int_{0}^{1} d y y^{n} \partial_{y} \chi_{121}(x, 1, y) & =\chi_{121}(x, 1,1)-\delta_{n, 0} \chi_{121}(x, 1,0) \\
& -n \int_{0}^{1} d y y^{n-1} \chi_{121}(x, 1, y),
\end{aligned}
$$


where we have introduced a new $Y$-factor,

$$
Y_{2}=-\frac{1}{2}\left(1-\mu_{4}^{2}+\mu_{3}^{2}\right), \quad b_{2 ; 121}=\lambda\left(1, \mu_{3}^{2}, \mu_{4}^{2}\right),
$$

with special cases given by $\chi_{121}(x, 1,1)=\mu_{4}^{2}, \chi_{121}(x, 0,0)=\mu_{x}^{2}$ and also $\chi_{121}(x, 1,0)=\mu_{3}^{2}$. Finally we have to consider terms containing $\chi_{121}(x, y, 0)$ which give

$$
\begin{aligned}
\ln \chi_{121}(x, y, 0) & =\chi_{121}(x, y, 0) \ln \chi_{121}(x, y, 0)-\left[1-\chi_{121}(x, y, 0)\right] \partial_{y} \frac{\chi_{121}(x, y, 0)}{b_{3 ; 121}} \\
& +\left[1-\chi_{121}(x, y, 0)\right] \partial_{y}\left[\frac{\chi_{121}(x, y, 0)}{b_{3 ; 121}} \ln \chi_{121}(x, y, 0)\right], \\
\ln ^{2} \chi_{121}(x, y, 0) & =-2\left\{-\frac{1}{2} \chi_{121}(x, y, 0) \ln ^{2} \chi_{121}(x, y, 0)-\left[1-\chi_{121}(x, y, 0)\right] \partial_{y} \frac{\chi_{121}(x, y, 0)}{b_{3 ; 121}}\right. \\
& +\left[1-\chi_{121}(x, y, 0)\right] \partial_{y}\left[\frac{\chi_{121}(x, y, 0)}{b_{3 ; 121}} \ln \chi_{121}(x, y, 0)\right] \\
& \left.-\frac{1}{2}\left[1-\chi_{121}(x, y, 0)\right] \partial_{y}\left[\frac{\chi_{121}(x, y, 0)}{b_{3 ; 121}} \ln ^{2} \chi_{121}(x, y, 0)\right]\right\},
\end{aligned}
$$

where the special case is $\chi_{121}(x, y, 0)=y \mu_{3}^{2}+(1-y) \mu_{x}^{2}$. For this combination we use

$$
\begin{aligned}
\int_{0}^{1} d y y^{n} \partial_{y} \chi_{121}(x, y, 0) \ln ^{m} \chi_{121}(x, y, 0) & =\chi_{121}(x, 1,0) \ln ^{m} \chi_{121}(x, 1,0) \\
& -\delta_{n, 0} \chi_{121}(x, 0,0) \ln ^{m} \chi_{121}(x, 0,0) \\
& -n \int_{0}^{1} d y y^{n-1} \chi_{121}(x, y, 0) \ln ^{m} \chi_{121}(x, y, 0), \\
\int_{0}^{1} d y y^{n} \partial_{y} \chi_{121}(x, y, 0)=\chi_{121}(x, 1,0) & -\delta_{n, 0} \chi_{121}(x, 0,0)-n \int_{0}^{1} d y y^{n-1} \chi_{121}(x, y, 0) .
\end{aligned}
$$

Here we have defined

$$
b_{3 ; 121}=\mu_{3}^{2}-\mu_{x}^{2} .
$$

For terms involving $\chi_{121}(x, y, z)$ the second step will be as follows:

$$
\begin{aligned}
\ln \chi_{121}(x, y, z) & =-\frac{4}{b_{121}}\left\{y \partial_{y} \chi_{121}(x, y, z)\left[1-\ln \chi_{121}(x, y, z)\right]\right. \\
& +\frac{1}{2}\left(z+Y_{2}\right) \partial_{z} \chi_{121}(x, y, z)\left[1-\ln \chi_{121}(x, y, z)\right] \\
& \left.+\chi_{121}(x, y, z) \ln \chi_{121}(x, y, z)\right\}
\end{aligned}
$$




$$
\begin{aligned}
\ln ^{2} \chi_{121}(x, y, z) & =\frac{2}{b_{121}}\left\{y \partial_{y} \chi_{121}(x, y, z)\left[1-\ln \chi_{121}(x, y, z)+\frac{1}{2} \ln ^{2} \chi_{121}(x, y, z)\right]\right. \\
& +\frac{1}{2}\left(z+Y_{2}\right) \partial_{z} \chi_{121}(x, y, z)\left[1-\ln \chi_{121}(x, y, z)+\frac{1}{2} \ln ^{2} \chi_{121}(x, y, z)\right] \\
& \left.-\frac{1}{2} \chi_{121}(x, y, z) \ln ^{2} \chi_{121}(x, y, z)\right\}
\end{aligned}
$$

Integration by parts can now be performed according to

$$
\begin{aligned}
\int_{0}^{y} d z z^{n} \partial_{z} \chi_{121}(x, y, z) \ln ^{m} \chi_{121}(x, y, z) & =y^{n} \chi_{121}(x, y, y) \ln ^{m} \chi_{121}(x, y, y) \\
& -\delta_{n, 0} \chi_{121}(x, y, 0) \ln ^{m} \chi_{121}(x, y, 0) \\
& -n \int_{0}^{y} d z z^{n-1} \chi_{121}(x, y, z) \ln ^{m} \chi_{121}(x, y, z), \\
\int_{0}^{y} d z z^{n} \partial_{z} \chi_{121}(x, y, z)=y^{n} \chi_{121}(x, y, y) & -\delta_{n, 0} \chi_{121}(x, y, 0)-n \int_{0}^{y} d z z^{n-1} \chi_{121}(x, y, z) .
\end{aligned}
$$

Furthermore, we exchange to order of integration,

$$
\int_{0}^{1} d y \int_{0}^{y} d z \partial_{y}=\int_{0}^{1} d z \int_{z}^{1} d y \partial_{y}
$$

to perform the remaining integration by parts according to

$$
\begin{aligned}
\int_{z}^{1} d y y^{n} \partial_{y} \chi_{121}(x, y, z) \ln ^{m} \chi_{121}(x, y, z) & =\chi_{121}(x, 1, z) \ln ^{m} \chi_{121}(x, 1, z) \\
& -z^{n} \chi_{121}(x, z, z) \ln ^{m} \chi_{121}(x, z, z) \\
& -n \int_{z}^{1} d y y^{n-1} \chi_{121}(x, y, z) \ln ^{m} \chi_{121}(x, y, z), \\
\int_{z}^{1} d y y^{n} \partial_{y} \chi_{121}(x, y, z) & =\chi_{121}(x, 1, z)-z^{n} \chi_{121}(x, z, z) \\
& -n \int_{z}^{1} d y y^{n-1} \chi_{121}(x, y, z) .
\end{aligned}
$$

To summarize, the following quadratic forms will appear in the final result as arguments of the logarithms:

$$
\begin{aligned}
& X_{121}(x, y, z)=x(1-x)\left[(y-z) \mu_{3}^{2}+z \mu_{4}^{2}-z(1-z)\right]+(1-y)\left[(1-x) \mu_{1}^{2}+x \mu_{2}^{2}\right], \\
& X_{121}(x, y, y)=x(1-x)\left[y \mu_{4}^{2}-y(1-y)\right]+(1-y)\left[(1-x) \mu_{1}^{2}+x \mu_{2}^{2}\right], \\
& X_{121}(x, y, 0)=x(1-x) y \mu_{3}^{2}+(1-y)\left[(1-x) \mu_{1}^{2}+x \mu_{2}^{2}\right], \\
& X_{121}(x, 1, y)=(1-y) \mu_{3}^{2}+y \mu_{4}^{2}-y(1-y) .
\end{aligned}
$$


Collecting the various terms we obtain a final answer that can be cast into the following form:

$$
S^{121}=\int_{0}^{1} d x \int_{0}^{1} d y\left\{\int_{0}^{y} d z \sum_{n=0}^{2} \frac{I_{3 n}}{\lambda_{0}^{n}}+\left[\sum_{n=0}^{2} \frac{I_{2 n}}{\lambda_{0}^{n}}+\frac{1}{\lambda_{0}} \sum_{n=1}^{3} \frac{I_{21 n}}{\lambda_{n}}+\sum_{n=1}^{3} \frac{I_{20 n}}{\lambda_{n}}\right]\right\} .
$$

Since we are not dealing with an analytical result and given that the number of terms in Eq.(100) is $\mathcal{O}\left(1.6 \times 10^{3}\right)$ we will not present its final explicit form in this paper.

\subsubsection{Zeros of b-functions}

In the final result for $S^{121}$ we encounter negative powers of four different $b$-functions whose zeros are as follows:

$-b_{121}$ of Eq.411). There are two roots for $x$, i.e.

$$
x_{ \pm}^{0}=\frac{2}{\mu_{34}^{4}}\left[\mu_{1}^{2}-\mu_{2}^{2}+\frac{1}{4} \mu_{34}^{4} \pm \lambda^{1 / 2}\left(\frac{\mu_{34}^{4}}{4}, \mu_{1}^{2}, \mu_{2}^{2}\right)\right],
$$

where $\mu_{34}^{2}=1+\mu_{3}^{2}-\mu_{4}^{2}$. These roots are real and internal to the interval $[0,1]$ if $\mu_{34}^{4} \leq$ $4\left(\mu_{1}-\mu_{2}\right)^{2}$ or $\mu_{34}^{4} \geq 4\left(\mu_{1}+\mu_{2}\right)^{2}$.

$-b_{1 ; 121}$ of Eq.(86). There are three possibilities:

1. $\mu_{1}+\mu_{2}-\mu_{4} \geq 1$, therefore $b_{1 ; 121}$ can never be zero.

2. $\left(1-\mu_{4}\right)^{2} \leq\left(\mu_{1}+\mu_{2}\right)^{2} \leq\left(1+\mu_{4}\right)^{2}$, when there are two values of $x$ where $b_{1 ; 121}=0$,

$$
x_{ \pm}^{1+}=\frac{1}{2\left(1+\mu_{4}\right)^{2}}\left[\left(1+\mu_{4}\right)^{2}+\mu_{1}^{2}-\mu_{2}^{2} \pm \lambda^{1 / 2}\left(\left(1+\mu_{4}\right)^{2}, \mu_{1}^{2}, \mu_{2}^{2}\right)\right] .
$$

3. $\left(\mu_{1}+\mu_{2}\right)^{2} \leq\left(1-\mu_{4}\right)^{2}$, when we have four values of $x$ where $b_{1 ; 121}=0$. The new pair of points is given by

$$
x_{ \pm}^{1-}=\frac{1}{2\left(1-\mu_{4}\right)^{2}}\left[\left(1-\mu_{4}\right)^{2}+\mu_{1}^{2}-\mu_{2}^{2} \pm \lambda^{1 / 2}\left(\left(1-\mu_{4}\right)^{2}, \mu_{1}^{2}, \mu_{2}^{2}\right)\right] .
$$

- $b_{2 ; 121}$ of Eq.(89). This is $x$-independent and vanishes for $s=\left(m_{3}-m_{4}\right)^{2}$ or $s=\left(m_{3}+\right.$ $\left.m_{4}\right)^{2}$. The latter corresponds to the normal threshold relative to the two-particle cut of the diagram [13].

$-b_{3 ; 121}$ of Eq.(93). There are two roots for $x$, i.e.

$$
x_{ \pm}^{3}=\frac{1}{2 \mu_{3}^{2}}\left[\mu_{3}^{2}+\mu_{1}^{2}-\mu_{2}^{2} \pm \lambda^{1 / 2}\left(\mu_{3}^{2}, \mu_{1}^{2}, \mu_{2}^{2}\right)\right]
$$

These roots are real and internal to the interval $[0,1]$ if $\mu_{3}^{2} \leq\left(\mu_{1}-\mu_{2}\right)^{2}$ or $\mu_{3}^{2} \geq\left(\mu_{1}+\mu_{2}\right)^{2}$. 


\subsubsection{The logarithms}

To discuss the logarithms and their branch points we write

$$
\begin{gathered}
\ln X_{121}(x, y, z)=\ln \left(a x^{2}+b x+c-i \delta\right), \\
a=z(1-z)-(y-z) \mu_{3}^{2}-z \mu_{4}^{2}, \quad b=-a+(1-y)\left(\mu_{2}^{2}-\mu_{1}^{2}\right), \quad c=(1-y) \mu_{1}^{2} .
\end{gathered}
$$

Let us denote the two roots of the quadratic by $x_{L, R}^{0}$. There are three sub-cases to be discussed,

- $X_{121}(x, y, y)$ where $a=y(1-y)-y \mu_{4}^{2}$ and with roots $x_{L, R}^{1}$,

- $X_{121}(x, 1, y)$, where $a=y(1-y)-(1-y) \mu_{3}^{2}-y \mu_{4}^{2}, b=-a$ and $c=0$, with roots $x_{L, R}^{2}$,

- $X_{121}(x, y, 0)$ where $a=-y \mu_{3}^{2}$, and with roots $x_{L, R}^{3}$.

\subsection{Evaluation of $S^{121}$ : methods III and IV}

$S^{121}$ can be cast into different forms, all suitable for numerical integration. Let us consider again Eq.(35) and change variable, $y=1-y^{\prime}$. We obtain

$$
\begin{aligned}
S^{121} & =-2\left(\frac{\mu^{2}}{\pi s}\right)^{\epsilon} \Gamma(\epsilon) \int_{0}^{1} d x \int_{0}^{1} d y \int_{0}^{1-y} d z[x(1-x)]^{-\epsilon / 2} y^{\epsilon / 2} \\
& \times\left[\left(\mu_{x}^{2}-z^{2}\right) \chi_{121}^{-1-\epsilon}+\frac{2-\epsilon / 2}{\epsilon} \chi_{121}^{-\epsilon}\right]
\end{aligned}
$$

where the quadratic form $\chi_{121}$ is now given by

$$
\chi_{121}(x, 1-y, z)=z^{2}-\mu_{34}^{2} z+\left(\mu_{x}^{2}-\mu_{3}^{2}\right) y+\mu_{3}^{2} .
$$

At this point we apply a special differential relation:

$$
\begin{aligned}
\left(\mu_{x}^{2}-\mu_{3}^{2}\right) \chi_{121}^{-1-\epsilon} & =-\frac{1}{\epsilon} \partial_{y} \chi_{121}^{-\epsilon}, \\
{\left[1+\frac{y}{\epsilon} \partial_{y}+\frac{1}{2 \epsilon}\left(z-\frac{1}{2} \mu_{34}^{2}\right) \partial_{z}\right] \chi_{121}^{-\epsilon} } & =-\frac{1}{4} b_{121}^{\prime} \chi_{121}^{-1-\epsilon},
\end{aligned}
$$

where now $b_{121}^{\prime}$ has the advantage of being $x$-independent, namely we get

$$
b_{121}^{\prime}=\lambda\left(1, \mu_{3}^{2}, \mu_{4}^{2}\right) .
$$

This second method is closer to the original BT approach in the sense that the denominator, after the raising procedure, is a function of the external parameters but does not depend on residual Feynman parameters. The zeros of $b_{121}^{\prime}$, Eq.(110), are the normal and pseudo thresholds corresponding to the two-particle cut of Fig. 5. They are non-leading Landau singularities. The 
fact that $b_{121}^{\prime}$ is $x$ independent means that, with this method, we can perform the $x$-integration without having to distort the integration contour. After integration by parts we obtain

$$
\begin{aligned}
S_{\text {fin }}^{121} & =-2\left\{\frac{1}{b_{121}^{\prime}}\left[\int_{0}^{1} d x \int_{0}^{1} d y\left(\int_{0}^{1-y} d z J_{31}^{121}+J_{21}^{121}\right)-\frac{1}{2} \mu_{3}^{2}-\frac{1}{72}\right]\right. \\
& \left.\left.+\int_{0}^{1} d x \int_{0}^{1} d y\left(\int_{0}^{1-y} d z J_{30}^{121}+J_{20}^{121}\right)+\frac{5}{8}+\frac{1}{4} \zeta(2)\right]\right\}
\end{aligned}
$$

where the various integrands are as follows:

$$
\begin{aligned}
J_{31}^{121} & =2\left(\mu_{3}^{2}-z^{2}\right) \ln \chi_{121}(x, 1-y, z)-\left(3 \mu_{3}^{2}+\mu_{34}^{2} z-5 z^{2}\right) \ln \chi_{121}(x, 1-y, z) \mathcal{L}_{-}(x, y, z), \\
J_{21}^{121} & =\left[-\frac{1}{2} \mu_{34}^{2} \mu_{3}^{2}+\frac{1}{2} \mu_{34}^{2}+\mu_{3}^{2}-1+\left(1-\mu_{34}^{2}+\mu_{3}^{2}\right) y+\left(1+\frac{1}{2} \mu_{34}^{2}\right) y^{2}-y^{3}\right] \\
& \times \ln \chi_{121}(x, 1-y, 1-y) \mathcal{L}_{-}(x, y, 1-y)+\frac{1}{2} \mu_{3}^{2} \mu_{34}^{2} \ln \chi_{121}(x, 1-y, 0) \mathcal{L}_{-}(x, y, 0), \\
J_{30}^{121} & =\ln \chi_{121}(x, 1-y, z)\left[\frac{1}{2}-\mathcal{L}_{-}(x, y, z)\right]-\frac{1}{2}\left(\frac{\ln \chi_{121}(x, 1-y, z)}{y}\right)_{+} \\
J_{20}^{121} & =\frac{1}{2}\left[\ln \chi_{121}(x, 1-y, 1-y) \mathcal{L}_{-}(x, y, 1-y)+\ln \chi_{121}(x, 1, y) \mathcal{L}_{+}(x, 1, y)\right] .
\end{aligned}
$$

In Eq.(112) we have introduced special combinations of logarithms,

$$
\begin{aligned}
& \mathcal{L}_{-}(x, y, z)=\ln y-\ln x-\ln (1-x)-\ln \chi_{121}(x, 1-y, z), \\
& \mathcal{L}_{+}(x, 1, y)=\ln \chi_{121}(x, 1, y)+\ln x+\ln (1-x)-\ln (1-y) .
\end{aligned}
$$

If requested, the procedure can be iterated without introducing numerical instabilities. However, the method fails at the non-leading Landau singularities corresponding to the one-loop sub-diagram with the largest number of internal lines. Therefore, in these regions we have to modify the procedure. Here, $\mu_{34}^{2}= \pm 2 \mu_{3}$ and $\chi_{121}=\left(z \mp \mu_{3}\right)^{2}+\left(\mu_{x}^{2}-\mu_{3}^{2}\right) y$; correspondingly we change variable, $z^{\prime}=z \mp \mu_{3}$ and obtain

$$
\int_{0}^{1} d y \int_{\mp \mu_{3}}^{1 \mp \mu_{3}-y} d z y^{\epsilon / 2}\left[\left(\mu_{x}^{2}-\left(z \pm \mu_{3}\right)^{2}\right) \chi_{121}^{-1-\epsilon}+\frac{2-\epsilon / 2}{\epsilon} \chi_{121}^{-\epsilon}\right], \quad \chi_{121}=z^{2}+\left(\mu_{x}^{2}-\mu_{3}^{2}\right) y .
$$

After a new change of variable, $z^{2}=t$, we use $M(t, y)=t+\left(\mu_{x}^{2}-\mu_{3}^{2}\right) y$ to derive

$$
\left\{\left(\mu_{x}^{2}-\mu_{3}^{2}\right) ; 1\right\} M^{-1-\epsilon}=\left\{\partial_{y} ; \partial_{z}\right\}\left[\ln M-\frac{\epsilon}{2} \ln ^{2} M+\mathcal{O}\left(\epsilon^{2}\right)\right]
$$

and arrive at the final result. For the $S^{121}$ diagram we have also another method (IV) which makes use of the following general property:

$$
\left(\mu_{x}^{2}-z^{2}\right) \chi_{121}^{-1-\epsilon}(x, y, z)=\left[1+\frac{1}{\epsilon}\left(y \partial_{y}+z \partial_{z}\right)\right] \chi_{121}^{-\epsilon}(x, y, z) .
$$




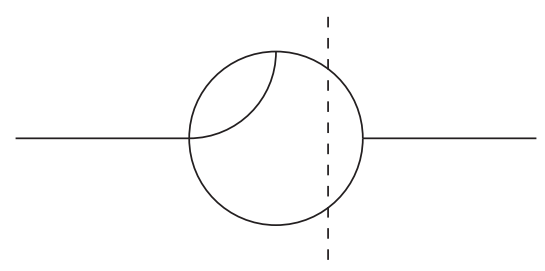

Figure 5: The two-particle cut of diagram $S^{121}$ of Fig. 2

After integration by parts we obtain:

$$
\begin{aligned}
S_{\mathrm{fin}}^{121} & =-\left\{\int_{0}^{1} d x \int_{0}^{1} d y \int_{0}^{y} d z\left(\frac{\ln \chi_{121}(x, y, z)}{y-1}\right)_{+}\right. \\
& \left.-\int_{0}^{1} d x \int_{0}^{1} d y \ln \chi_{121}(x, 1, y) L_{1}(x, 1, y)+\frac{3}{2}+\frac{1}{2} \zeta(2)\right\},
\end{aligned}
$$

with $L_{1}$ given by

$$
L_{1}(x, y, z)=\ln (1-y)-\ln x-\ln (1-x)-\ln \chi_{121}(x, y, z) .
$$

Method IV represents the simplest results for $S^{121}$ but it is not generalizable to more complex topologies.

\subsection{Derivative of $S^{121}$ and infrared poles.}

The approach described in this paper is primarily intended for evaluation of massive multi-loop diagrams. However, QED and QCD will be part of any realistic calculation and they usually lead to infrared singularities. Any method aimed to a numerical evaluation of diagrams must be able to handle the infrared problem. For one-loop diagrams we have seen that the method is able to extract the infrared pole in dimensional regularization with a residue and a finite part that can be treated numerically [7]. We have to extend the treatment of infrared divergences to multi-loop and we will start the discussion with one specific example: we define the on-shell derivative of a two-point function, where possibly some of the internal masses are zero, as the $\partial / \partial p^{2}$ derivative evaluated at the mass shell of one of the non-zero internal masses. This paper concerns infrared configurations only and the general case will be presented together with vertices [15.

Consider now the on-shell derivative of $S^{121}$. Indeed, for the simplest topology $S^{111}$ the on-shell derivative is infrared finite, see Appendix A.

It is important to recall that a necessary condition for the presence of infrared divergences is that the Landau equations are fulfilled. In the case of $S^{121}$ we see that $s=\left(m_{1}+m_{2} \pm m_{4}\right)^{2}$ and $m_{3}^{2}=\left(m_{1}+m_{2}\right)^{2}$ are satisfied by $m_{2}=m_{4}=0, m_{1}=m_{3}=m$ and $s=m^{2}$. However $S^{121}$ itself is not infrared divergent but self-energy diagrams enter into the calculation of wave function renormalization factor and, therefore, also their derivative with respect to $p^{2}$ is needed. This derivative, as in the one-loop case, shows an infrared pole when computed on-shell. Therefore, we 
consider the case $m_{2}=m_{4}=0$ and $m_{1}=m_{3}=m$, a typical example of which is shown in Fig. 6. For these values of the internal masses we get

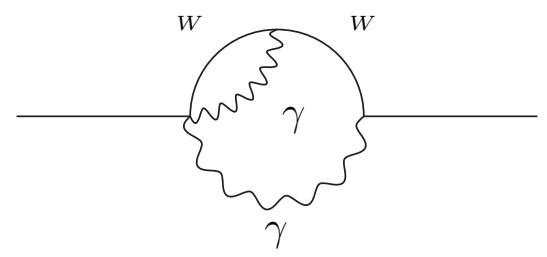

Figure 6: A two-loop diagram contribution to the $W$-boson self-energy.

$$
\begin{aligned}
S^{121} & =-2\left(\frac{\mu^{2}}{\pi}\right)^{\epsilon} \Gamma(\epsilon) \int_{0}^{1} d x \int_{0}^{1} d y \int_{0}^{y} d z[x(1-x)]^{-\epsilon / 2}(1-y)^{\epsilon / 2} \\
& \times\left[\left(m_{x}^{2}+z^{2} p^{2}\right) U_{121}^{-1-\epsilon}+\frac{2-\epsilon / 2}{\epsilon} U_{121}^{-\epsilon}\right]
\end{aligned}
$$

where the quadratic form $U_{121}$ is

$$
U_{121}=-p^{2} z^{2}+\left(p^{2}-m^{2}\right) z+m^{2}\left(1-\frac{1}{x}\right) y+\frac{m^{2}}{x} .
$$

First we take the derivative of $S^{121}$ with respect to $p^{2}$ and next we go on-shell by putting $p^{2}=-m^{2}$.

\subsubsection{On-shell derivative of $S^{121}$ : method I}

Proceeding as described above we obtain the derivative as

$$
\begin{aligned}
S_{p}^{121} & =-\frac{2}{m^{2}}\left(\frac{\mu^{2}}{\pi m^{2}}\right)^{\epsilon} \Gamma(\epsilon) \int_{0}^{1} d x \int_{0}^{1} d y \int_{0}^{y} d z[x(1-x)]^{-\epsilon / 2}(1-y)^{\epsilon / 2} \\
& \times\left.\left\{z(1-z)\left[(1+\epsilon)\left(z^{2}-\frac{1}{x}\right) \chi_{121}^{-2-\epsilon}+\frac{\epsilon}{2} \chi_{121}^{-1-\epsilon}\right]+z(3 z-2) \chi_{121}^{-1-\epsilon}\right\}\right|_{\mathrm{os}},
\end{aligned}
$$

where we have introduced

$$
\chi_{\mathrm{os} ; 121}=(z-1)^{2}+\frac{1}{X}(1-y), \quad X=\frac{x}{1-x} .
$$

We introduce new variables $y=1-y^{\prime}$ and $z=1-z^{\prime}$, so that

$$
U_{121}^{\mathrm{os}}=m^{2} \chi_{\mathrm{os} ; 121}=m^{2}\left(z^{2}+\frac{y}{X}\right)
$$

and consider the term

$$
\left[(1-z)^{2}-\frac{1}{x}\right]\left(\chi_{\mathrm{os} ; 121}\right)^{-2-\epsilon}
$$


To go further, we can use the following relation:

$$
\begin{gathered}
\mathcal{D}_{i}\left(z^{2}+\frac{y}{X}\right)^{-1-\epsilon}=(1+\epsilon) d_{i}\left(z^{2}+\frac{y}{X}\right)^{-2-\epsilon}, \\
\mathcal{D} \equiv\left(\partial_{y}-\frac{z}{2} \partial_{z}, \partial_{z}, \partial_{y}\right), \quad d \equiv\left(z^{2}-\frac{1}{X},-2 z,-\frac{1}{X}\right) .
\end{gathered}
$$

After integration by parts we use

$$
\left(\partial_{z} ; \partial_{y}\right)\left(z^{2}+\frac{y}{X}\right)^{-\epsilon}=\epsilon\left(-2 x ;-\frac{1}{X}\right)\left(z^{2}+\frac{y}{X}\right)^{-1-\epsilon} .
$$

In this way we derive

$$
S_{p}^{121}=-\frac{2}{m^{2}}\left(\frac{\mu^{2}}{\pi m^{2}}\right)^{\epsilon} \Gamma(\epsilon) \int_{0}^{1} d x[x(1-x)]^{-\epsilon / 2} \mathcal{S},
$$

where $\mathcal{S}$ is a combination of one and two dimensional integrals. Due to the presence of an infrared pole in the on-shell derivative we must keep $\epsilon \neq 0$. Next we change variable, $y=z y^{\prime}$, and introduce an auxiliary integral

$$
I(\mu, \nu)=\int_{0}^{1} d y \int_{0}^{1} d z y^{\mu} z^{\nu}\left(z+\frac{y}{X}\right)^{-\epsilon} .
$$

Using the relation

$$
\left[1+\frac{1}{\epsilon}\left(y \partial_{y}+z \partial_{z}\right)\right]\left(z+\frac{y}{X}\right)^{-\epsilon}=0
$$

we are able to derive

$$
I(\mu, \nu)=\frac{1}{2+\mu+\nu-\epsilon} \int_{0}^{1} d y\left[y^{\mu}\left(1+\frac{y}{X}\right)^{-\epsilon}+y^{\nu}\left(y+\frac{1}{X}\right)^{-\epsilon}\right] .
$$

In this way the evaluation of $\mathcal{S}$ is simply reduced to the calculation of a one-dimensional integral which can be cast into the following form:

$$
\mathcal{S}=\int_{0}^{1} d y \mathcal{S}_{y}
$$

with an integrand of the form

$$
\begin{aligned}
\mathcal{S}_{y} & =\left[-\frac{1}{4} y^{-1-\epsilon / 2}+\frac{1}{4}\left(\frac{4}{\epsilon}-2+\frac{3}{3-\epsilon}\right) y^{1-\epsilon / 2}+\frac{1}{4}\left(3-\frac{2}{\epsilon}+\frac{1}{1-\epsilon}-\frac{3}{3-\epsilon}\right) y^{-\epsilon / 2}\right](1+X y)^{-\epsilon} \\
& +\frac{1}{2} X^{1+\epsilon}\left(y^{1-\epsilon / 2}-y^{2-\epsilon / 2}\right)(1+X y)^{-1-\epsilon}+\frac{1}{4} \frac{1}{1-\epsilon} y^{-1+\epsilon / 2}\left(1+\frac{y}{X}\right)^{-\epsilon} .
\end{aligned}
$$

First we consider the integrals that produce a pole at $\epsilon=0$, therefore

$$
\int_{0}^{1} d y y^{ \pm \epsilon / 2-1}(1+\alpha y)^{-\epsilon}= \pm \frac{2}{\epsilon}-\epsilon \int_{0}^{1} d y \frac{\ln (1+\alpha y)}{y}+\mathcal{O}\left(\epsilon^{2}\right), \quad \alpha=X, X^{-1} .
$$


Successively we multiply the result by $[x(1-x)]^{-\epsilon}$ and expand around $\epsilon=0$. The remaining integrals can be computed in terms of a long list of master integrals that will be reported in Appendix B. The $x$-integration gives

$$
\int_{0}^{1} d x[x(1-x)]^{-\epsilon / 2} \mathcal{S}=S_{-1} \epsilon^{-1}+S_{0}+S_{1} \epsilon
$$

with coefficients

$$
S_{-1}=1, \quad S_{0}=2, \quad S_{1}=\frac{7}{2}-\frac{1}{4} \zeta(2) .
$$

To obtain our final result we have used the following relation:

$$
\operatorname{Li}_{2}\left(-\frac{1}{X}\right)=\operatorname{Li}_{2}(x)+\ln x \ln (1-x)-\frac{1}{2} \ln ^{2} x-\zeta(2),
$$

and the following integrals:

$$
\int_{0}^{1} d x\left\{\frac{\ln (1-x)}{x} ; \operatorname{Li}_{2}(x) ; \ln ^{2}(1-x) ; \ln (1-x)\right\}=\{-\zeta(2) ; \zeta(2)-1 ; 2 ;-1\} .
$$

The final result for the on-shell derivative reads as follows:

$$
S_{p}^{121}=\frac{1}{m^{2}}\left(\frac{\mu^{2}}{\pi m^{2}}\right)^{\epsilon}\left[-\frac{2}{\epsilon^{2}}-2(2-\gamma) \frac{1}{\epsilon}-7+\gamma(4-\gamma)-\frac{1}{2} \zeta(2)+\mathcal{O}(\epsilon)\right]
$$

\subsubsection{On-shell derivative of $S^{121}$ : method II}

An alternative derivation of the on-shell derivative of $S^{121}$ will also be presented. We can use the following relation

$$
\left[1+\frac{1}{1+\epsilon}\left(y \partial_{y}+z \partial_{z}\right)\right] \chi_{\mathrm{os} ; 121}^{-1-\epsilon}=-\left(z^{2}-\frac{1}{x}\right) \chi_{\mathrm{os} ; 121}^{-2-\epsilon},
$$

and derive an alternative result

$$
S_{p}^{121}=\frac{\Gamma(1+\epsilon)}{m^{2}}\left(\frac{\mu^{2}}{\pi m^{2}}\right)^{\epsilon} \int_{0}^{1} d x \int_{0}^{1} d y \int_{0}^{y} d z[x(1-x)]^{-\epsilon / 2}(1-y)^{\epsilon / 2-1} z(1-z) \chi_{\mathrm{os} ; 121}^{-1-\epsilon}
$$

Instead of changing variables we can use another important identity,

$$
z(1-z) \chi_{\mathrm{os} ; 121}^{-1-\epsilon}=\frac{1}{2 \epsilon} z \partial_{z} \chi_{\mathrm{os} ; 121}^{-\epsilon}
$$

Once this relation is applied and the integration by parts performed we introduce new variables, $y=1-y^{\prime}$ and $z=1-z^{\prime}$, so that

$$
S_{p}^{121}=\frac{1}{2 m^{2}}\left(\frac{\mu^{2}}{\pi m^{2}}\right)^{\epsilon} \Gamma(\epsilon) \int_{0}^{1} d x[x(1-x)]^{-\epsilon / 2}\left(\mathcal{S}_{1}-\mathcal{S}_{2}\right)
$$




$$
\mathcal{S}_{1}=\int_{0}^{1} d y(1-y) y^{-\epsilon / 2-1}\left(y+X^{\prime}\right)^{-\epsilon}, \quad \mathcal{S}_{2}=\int_{0}^{1} d y \int_{y}^{1} d z y^{\epsilon / 2-1}\left(z^{2}+X^{\prime} y\right)^{-\epsilon},
$$

where we used $X^{\prime}=X^{-1}=1 / x-1$. Next we change variable, $y=z y^{\prime}$, and utilize the auxiliary integral $I(\mu, \nu)$ of Eq.(131), which gives for $\mathcal{S}_{2}$ the following result:

$$
\mathcal{S}_{2}=\frac{1}{1-\epsilon} \int_{0}^{1} d y\left[y^{\frac{\epsilon}{2}-1}\left(1+X^{\prime} y\right)^{-\epsilon}+y^{-\frac{\epsilon}{2}}\left(y+X^{\prime}\right)^{-\epsilon}\right]
$$

From well-known properties of the hypergeometric function [16] we get

$$
\begin{aligned}
\mathcal{S}_{1} & =-\frac{2}{\epsilon} X_{2}{ }_{2} F_{1}\left(\epsilon,-\frac{\epsilon}{2} ; 1-\frac{\epsilon}{2} ;-X\right)-\frac{2}{2-\epsilon} X^{\epsilon}{ }_{2} F_{1}\left(\epsilon, 1-\frac{\epsilon}{2} ; 2-\frac{\epsilon}{2} ;-X\right) \\
& =-\frac{2}{\epsilon} x_{2}{ }_{2} F_{1}\left(\epsilon, 1 ; 1-\frac{\epsilon}{2} ; x\right)-\frac{2}{2-\epsilon} x^{\epsilon}{ }_{2} F_{1}\left(\epsilon, 1 ; 2-\frac{\epsilon}{2} ; x\right) \\
\mathcal{S}_{2} & =\frac{2}{\epsilon(1-\epsilon)}{ }_{2} F_{1}\left(\epsilon, \frac{\epsilon}{2} ; 1+\frac{\epsilon}{2} ;-X^{\prime}\right)+\frac{2}{(1-\epsilon)(2-\epsilon)} X^{\epsilon}{ }_{2} F_{1}\left(\epsilon, 1-\frac{\epsilon}{2} ; 2-\frac{\epsilon}{2} ;-X\right) \\
& =\frac{2}{\epsilon(1-\epsilon)} x^{\epsilon}{ }_{2} F_{1}\left(\epsilon, 1 ; 1+\frac{\epsilon}{2} ; 1-x\right)+\frac{2}{(1-\epsilon)(2-\epsilon)} x_{2}^{\epsilon} F_{1}\left(\epsilon, 1 ; 2-\frac{\epsilon}{2} ; x\right)
\end{aligned}
$$

After introducing the hypergeometric series, we are able to perform the $x$ integration explicitly. Finally we expand around $\epsilon=0$ by using:

$$
\Gamma(n+m)=\Gamma(n)\left\{1+\left(\sum_{j=1}^{n-1} \frac{1}{j}-\gamma\right) m+\mathcal{O}\left(m^{2}\right)\right\}
$$

and arrive at the result of Eq.(139). To obtain Eq.(139) in method II we have used the following series:

$$
\sum_{n=1}^{\infty} \frac{1}{n(n+1)}=1, \quad \sum_{n=1}^{\infty} \frac{1}{n(n+1)} \sum_{j=1}^{n} \frac{1}{j}=\zeta(2), \quad \sum_{n=1}^{\infty} \frac{1}{n^{2}(n+1)}=\zeta(2)-1 .
$$

\subsubsection{Another on-shell derivative of $S^{121}$}

There is another configuration of interest shown in Fig. 7

$$
\text { b) } \quad m_{3}=0 \text {, and } m_{i}=m, \forall i \neq 3 \text {, }
$$

which corresponds to the non-leading Landau singularity $\alpha_{1}=\alpha_{2}=0$ and $s=m^{2}$. We obtain

$$
S_{p b}^{121}=\left(\frac{\mu^{2}}{\pi}\right)^{\epsilon} \Gamma(\epsilon+1) \int_{0}^{1} d x \int_{0}^{1} d y \int_{0}^{1-y} d z[x(1-x)]^{-\epsilon / 2} y^{\epsilon / 2-1} z(1-z) U_{\mathrm{os} ; 121}^{-1-\epsilon}(x, y, z),
$$

where we have introduced

$$
U_{\mathrm{os} ; 21}(x, y, z)=m^{2} z^{2}+m_{x}^{2} y, \quad m_{x}^{2}=\frac{m^{2}}{x(1-x)} .
$$

The evaluation of $S_{p b}^{121}$ will be postponed till Section 7.4 where it will be discussed with other similar functions. 


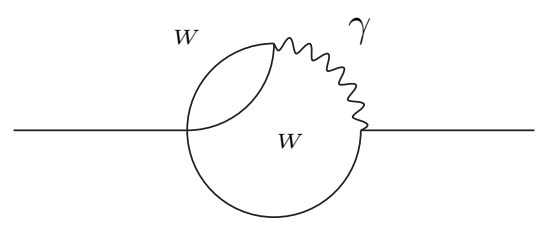

$W$

Figure 7: A two-loop diagram contribution to the $W$-boson self-energy.

\subsection{Tensor integrals of the $S^{121}$ family}

Let us define the following function:

$$
\pi^{4} S_{4}^{1-i / 2,2,1-j / 2}\left(\mu_{1} \ldots \mu_{i} \mid \nu_{1} \ldots \nu_{j}\right)=\mu^{2 \epsilon} \int d^{n} q_{1} d^{n} q_{2} \frac{q_{1}^{\mu_{1}} \ldots q_{1}^{\mu_{i}} q_{2}^{\nu_{1}} \ldots q_{2}^{\nu_{j}}}{\left(q_{1}^{2}+m_{1}^{2}\right) \ldots\left(\left(q_{2}+p\right)^{2}+m_{4}^{2}\right)},
$$

where we assume $i+j \leq 3$ and where $\alpha$ and $\beta$ have been changed in order to account for the effective degree of convergence. For tensor integrals we always indicate explicitly the total number of internal lines. In any realistic calculation the first step is about simplifying numerators and denominators as much as possible. For example we will use results as the following one:

$$
\begin{aligned}
& 2 \frac{\mu^{2 \epsilon}}{\pi^{4}} \int d^{n} q_{1} d^{n} q_{2} \frac{p \cdot q_{2}}{\left(q_{1}^{2}+m_{1}^{2}\right) \ldots\left(\left(q_{2}+p\right)^{2}+m_{4}^{2}\right)}=\left[S^{111}\left(0, m_{1}, m_{2}, m_{3}\right)\right. \\
- & \left.S^{111}\left(p^{2}, m_{1}, m_{2}, m_{4}\right)+\left(m_{3}^{2}-m_{4}^{2}-p^{2}\right) S_{4}^{121}\left(p^{2}, m_{1}, m_{2}, m_{3}, m_{4}\right)\right] .
\end{aligned}
$$

After possible simplifications few irreducible integrals, of the type appearing in Eq.(152), will remain. Their structure will be as follows:

$$
\begin{aligned}
& S_{4}^{1-i / 2,2,1-j / 2}(p \ldots p \mid p \ldots p)=\left(\frac{\mu^{2}}{\pi s}\right)^{\epsilon} \Gamma(\epsilon) \int_{0}^{1} d x \int_{0}^{1} d y \int_{0}^{y} d z \\
\times & {[x(1-x)]^{-\epsilon / 2}(1-y)^{\epsilon / 2} \sum_{n=-1}^{+1} Q_{n}^{i j}(\epsilon, x, y, z) \chi_{121}^{n-\epsilon}(x, y, z) . }
\end{aligned}
$$

The coefficients $Q$ are:

$$
\begin{array}{lll}
Q_{-1}^{10}=-2 x z\left(\mu_{x}^{2}-z^{2}\right) & Q_{0}^{10}=x z\left(1-\frac{6}{\epsilon}\right) & Q_{+1}^{10}=0 \\
Q_{-1}^{01}=-2 z\left(\mu_{x}^{2}-z^{2}\right) & Q_{0}^{01}=z\left(1-\frac{6}{\epsilon}\right) & Q_{+1}^{01}=0
\end{array}
$$

and also

$$
\begin{aligned}
Q_{-1}^{20} & =\left(\mu_{x}^{2}-z^{2}\right)\left[-2 x^{2} z^{2}+\frac{2}{\epsilon-2} x(1-x)\left(\mu_{x}^{2}-z^{2}\right)\right], \\
Q_{0}^{20} & =\left(\mu_{x}^{2}-z^{2}\right)\left[\frac{x^{2}}{\epsilon}-2 \frac{\epsilon-4}{\epsilon(\epsilon-2)} x(1-x)\right]+z^{2}\left[\left(1-\frac{8}{\epsilon}\right) x^{2}-4 \frac{x(1-x)}{\epsilon(\epsilon-2)}\right], \\
Q_{+1}^{20} & =-\left(\frac{3}{\epsilon}-\frac{5}{2} \frac{1}{\epsilon-1}\right)\left[x^{2}-x(1-x) \frac{\epsilon-4}{\epsilon-2}\right],
\end{aligned}
$$




$$
\begin{aligned}
& Q_{-1}^{02}=-2 z^{2}\left(\mu_{x}^{2}-z^{2}\right), \quad Q_{0}^{02}=z^{2}\left(1-\frac{8}{\epsilon}\right)+\left(\mu_{x}^{2}-z^{2}\right) \frac{1}{\epsilon}, \\
& Q_{+1}^{02}=-\frac{3}{\epsilon}+\frac{5}{2} \frac{1}{\epsilon-1}, \quad Q_{-1}^{11}=-2 x z^{2}\left(\mu_{x}^{2}-z^{2}\right), \\
& Q_{0}^{11}=x z^{2}\left(1-\frac{8}{\epsilon}\right)+x\left(\mu_{x}^{2}-z^{2}\right) \frac{1}{\epsilon}, \quad Q_{+1}^{11}=-x\left(\frac{3}{\epsilon}-\frac{5}{2} \frac{1}{\epsilon-1}\right) .
\end{aligned}
$$

Note that all terms proportional to $\chi_{121}^{-1-\epsilon}$, i.e. the $Q_{-1}^{i j}$ contain a factor $\mu_{x}^{2}-z^{2}$ which, in turn, allows us to raise the negative power through Eq.(49).

Several of the properties that we have shown for $S^{121}$ hold for a more general class of multi-loop diagrams, $G^{1 N 1}$ with $N \geq 2$.

\section{The $G^{1_{N} 1}$ topology}

$S^{121}$ is a special example of a general class of two-loop diagrams with $N+2$ internal lines which are overall convergent for $N>2$ and contain the $\alpha \gamma$ logarithmically divergent sub-diagram. We have

$$
\pi^{4} G^{1 N 1}=\mu^{2 \epsilon} \int d^{n} q_{1} d^{n} q_{2}\left(q_{1}^{2}+m_{1}^{2}\right)^{-1}\left(\left(q_{1}-q_{2}\right)^{2}+m_{2}^{2}\right)^{-1} \prod_{i=0}^{N-1}\left(\left(q_{2}+k_{i}\right)^{2}+m_{i+3}^{2}\right)^{-1}
$$

where the momenta $k_{i}$ are linear combinations of the external momenta $p_{j}, k_{0}=0$ and $k_{i}=$ $p_{1}+\ldots+p_{i}$. We introduce Feynman parameters $z_{i}$ for the propagators of the $q_{2}$ loop,

$$
d z_{L}=\prod_{i=0}^{N-1} d z_{i} \delta\left(1-z_{L}\right), \quad z_{L}=\sum_{i=0}^{N-1} z_{i}
$$

and also a $\left\{z_{i}\right\}$-dependent momentum and mass

$$
P_{\mu}=\sum_{i=0}^{N-1} z_{i} k_{i \mu}, \quad M^{2}=\sum_{i=0}^{N-1} z_{i}\left(k_{i}^{2}+m_{i+3}^{2}\right)
$$

and obtain

$$
G^{1 N 1}=-2\left(\frac{\mu^{2}}{\pi}\right)^{\epsilon} \frac{\Gamma(N-1+\epsilon)}{\epsilon} \int d[P]\left[\left(m_{x}^{2}+P^{2} y^{2}\right) \chi^{1-N-\epsilon}+\frac{1}{2} \frac{4-\epsilon}{N-2+\epsilon} \chi^{2-N-\epsilon}\right]
$$

where $x$ and $y$ parameters have been introduced to combine all propagators arising after the $q_{2}$ integration. Additional quantities are as follows:

$$
\begin{aligned}
\chi & =-P^{2} y^{2}+\left(M^{2}-m_{x}^{2}\right) y+m_{x}^{2} \\
\int d[P] & =\int_{0}^{1} d x \int_{0}^{1} d y \int d z_{L}[x(1-x)]^{-\epsilon / 2} y^{N-1}(1-y)^{\epsilon / 2}
\end{aligned}
$$


Next we change variables, $z_{i}=z_{i}^{\prime} / y$, and obtain

$$
\begin{aligned}
G^{1 N 1} & =-2\left(\frac{\mu^{2}}{\pi}\right)^{\epsilon} \frac{\Gamma(N-1+\epsilon)}{\epsilon} \int_{0}^{1} d x \int_{0}^{1} d y \int_{0}^{+\infty} \prod_{i=0}^{N-1} d z_{i}[x(1-x)]^{-\epsilon / 2}(1-y)^{\epsilon / 2} \delta\left(y-z_{L}\right) \\
& \times\left[\left(m_{x}^{2}-z^{t} H z\right) \chi^{1-N-\epsilon}+\frac{1}{2} \frac{4-\epsilon}{N-2+\epsilon} \chi^{2-N-\epsilon}\right]
\end{aligned}
$$

where the quadratic form $\chi$ has been rewritten as

$$
\chi=z^{t} H z+2 K^{t} z+L, \quad H_{i j}=-k_{i} \cdot k_{j}, \quad K_{i}=\frac{1}{2}\left(k_{i}^{2}+m_{i+3}^{2}\right), \quad L=m_{x}^{2}(1-y) .
$$

\section{The $S_{5}$ family}

There are two diagrams in the $S_{5}$ family, $S^{131}$ given in Fig. 8 and $S^{221}$, given in Fig. 12. $S^{131}$ is overall logarithmically divergent and contains the logarithmically divergent sub-diagram $\alpha \gamma$. $S^{221}$ and all its sub-diagrams are convergent. Their evaluation will be discussed in the following sections.

\section{$7.1 \quad S^{131}$ topology}

The $S^{131}$ topology, explicitly shown in Fig. 8, is given by

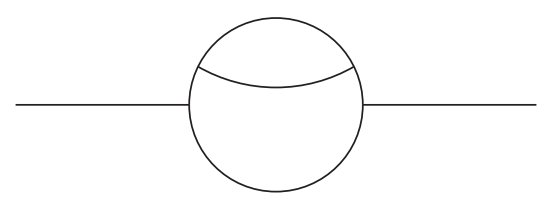

Figure 8: The two-loop diagram $S^{131}$ of Eq.(164).

$$
\pi^{4} S^{131}=\mu^{2 \epsilon} \int \frac{d^{n} q_{1} d^{n} q_{2}}{\left(q_{1}^{2}+m_{1}^{2}\right)\left(\left(q_{1}-q_{2}\right)^{2}+m_{2}^{2}\right)\left(q_{2}^{2}+m_{3}^{2}\right)\left(\left(q_{2}+p\right)^{2}+m_{4}^{2}\right)\left(q_{2}^{2}+m_{5}^{2}\right)} .
$$

If $m_{3} \neq m_{5}$ then $S^{131}$ is the difference of two $S^{121}$ diagrams,

$$
S^{131}=\frac{1}{m_{5}^{2}-m_{3}^{2}}\left[S^{121}\left(p^{2} ; m_{1}, m_{2}, m_{3}, m_{4}\right)-S^{121}\left(p^{2} ; m_{1}, m_{2}, m_{5}, m_{4}\right)\right],
$$

otherwise it is a special case of $G^{131}$ with $k_{0}=k_{2}=0$, and $k_{1}=-p$. The quadratic $\chi_{131}$ will be

$$
\chi_{131}=z^{2}-\left(1-\mu_{4}^{2}+\mu_{3}^{2}\right) z+\left(\mu_{3}^{2}-\mu_{x}^{2}\right) y+\mu_{x}^{2},
$$


where we have used $z_{2}=y-z_{1}-z_{0}$ and, moreover, $z \equiv z_{1}$. Limits of integration are $0 \leq z_{0} \leq y-z_{1}$ and $0 \leq z_{1} \leq y$. Therefore, for $m_{3}=m_{5}$ we obtain

$$
\begin{aligned}
S^{131} & =-\frac{2}{s}\left(\frac{\mu^{2}}{\pi s}\right)^{\epsilon} \frac{\Gamma(2+\epsilon)}{\epsilon} \int_{0}^{1} d x \int_{0}^{1} d y \int_{0}^{y} d z[x(1-x)]^{-\epsilon / 2}(1-y)^{\epsilon / 2}(y-z) \\
& \times\left[\left(\mu_{x}^{2}-z^{2}\right) \chi_{131}^{-2-\epsilon}+\frac{2-\epsilon / 2}{1+\epsilon} \chi_{131}^{-1-\epsilon}\right],
\end{aligned}
$$

The ultraviolet singularities can be easily derived by taking the derivative with respect to $m_{3}^{2}$ of $S^{121}$, therefore we obtain no double-pole, as expected, and a single pole given by

$$
-2\left(\frac{1}{\epsilon}-\Delta_{U V}\right) \frac{\partial}{\partial m_{3}^{2}} \int_{0}^{1} d x \int_{0}^{1} d y \ln \chi_{121}(x, 1, y)=-\frac{2}{s}\left(\frac{1}{\epsilon}-\Delta_{U V}\right) \int_{0}^{1} d y \frac{1-y}{\chi_{121}},
$$

Note that the residue of the single pole is what we expect in order to compensate the overlapping divergence coming from the associated subtraction diagram of Fig. 9. The integral can be computed, giving

$$
\begin{aligned}
\int_{0}^{1} d y \frac{1-y}{\chi_{121}} & =\frac{1}{2}\left\{\ln \frac{\mu_{3}^{2}}{\mu_{4}^{2}}+\frac{\mu_{43}^{2}}{\lambda^{1 / 2}\left(1, \mu_{3}^{2}, \mu_{4}^{2}\right)}\left[\ln \left(1-\frac{1}{y_{+}}\right)-\ln \left(1-\frac{1}{y_{-}}\right)\right]\right\} \\
y_{ \pm} & =\frac{1}{2}\left[\mu_{34}^{2} \pm \lambda^{1 / 2}\left(1, \mu_{3}^{2}, \mu_{4}^{2}\right)\right]
\end{aligned}
$$

where $\lambda \rightarrow \lambda+i \delta$ and $\delta \rightarrow 0_{+}$. If we introduce $y_{m}=1 / 2 \mu_{34}^{2}$ and $Y_{ \pm}=1-1 / y_{ \pm}$, then it follows that

$$
\ln Y_{+}-\ln Y_{-}=\ln \frac{Y_{+}}{Y_{-}}+\eta\left(Y_{-}, \frac{Y_{+}}{Y_{-}}\right), \quad \ln \frac{Y_{+}}{Y_{-}}=\lambda^{1 / 2}\left(1, \mu_{3}^{2}, \mu_{4}^{2}\right) \frac{1}{y_{m}\left(y_{m}-1\right)}+\mathcal{O}(\lambda) .
$$

Therefore, for $\lambda \rightarrow 0_{+}\left(0_{-}\right)$we observe a square root singularity in the imaginary(real) part. Recalling that the counter-term, in the MS-scheme, is $-2 i \pi^{2} / \epsilon$ and that the remaining integral is a $C_{0}$-function with $p_{1}=-p_{2}=p$ we obtain the following result for the subtraction diagram of Fig. 9:

$$
S^{\underline{1}} \underline{1}=\frac{2}{s \epsilon}\left[\int_{0}^{1} d y \frac{1-y}{\chi_{121}}+\mathcal{O}(\epsilon)\right] .
$$

\subsection{Landau equations for $S^{131}$ when $m_{5}=m_{3}$}

The Landau equations for this topology and with $m_{5}=m_{3}$ are as follows:

$$
\begin{array}{ll}
\alpha_{1}\left(q_{1}^{2}+m_{1}^{2}\right)=0, & \alpha_{2}\left(\left(q_{1}-q_{2}\right)^{2}+m_{2}^{2}\right)=0, \\
\alpha_{3}\left(q_{2}^{2}+m_{3}^{2}\right)=0, & \alpha_{4}\left(\left(q_{2}+p\right)^{2}+m_{4}^{2}\right)=0, \\
\alpha_{5}\left(q_{2}^{2}+m_{5}^{2}\right)=0, &
\end{array}
$$




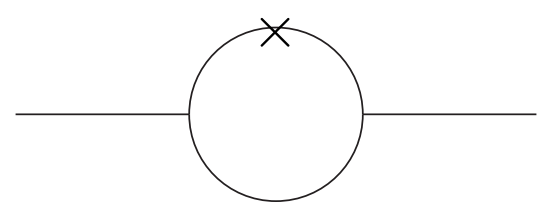

Figure 9: The subtraction diagram, containing a one-loop counter-term (represented by a $\times$ ) associated with the two-loop diagram $S^{131}$ of Fig. 8 .

and also

$$
\begin{aligned}
& \alpha_{1} q_{1 \mu}+\alpha_{2}\left(q_{1}-q_{2}\right)_{\mu}=0, \\
& -\alpha_{2}\left(q_{1}-q_{2}\right)_{\mu}+\alpha_{3} q_{2 \mu}+\alpha_{4}\left(q_{2}+p\right)_{\mu}+\alpha_{5} q_{2 \mu}=0 .
\end{aligned}
$$

The leading Landau singularity occurs for $\alpha_{i} \neq 0, \forall i$. We multiply the two equations Eq.(173) by $q_{1 \mu}, q_{2 \mu}$ and $p_{\mu}$ respectively. This gives an homogeneous system of six equations. If all $\alpha_{i}$ are different from zero, the singularity will occur for

$$
\begin{array}{ll}
q_{1}^{2}=-m_{1}^{2} & q_{2}^{2}=-m_{3}^{2}, \\
q_{1} \cdot q_{2}=\frac{1}{2}\left(m_{2}^{2}-m_{1}^{2}-m_{3}^{2}\right) & p \cdot q_{2}=\frac{1}{2}\left(s+m_{3}^{2}-m_{4}^{2}\right) .
\end{array}
$$

The equations become as follows:

$$
\begin{aligned}
& -2 m_{1}^{2} \alpha_{1}-\left(m_{1}^{2}+m_{2}^{2}-m_{3}^{2}\right) \alpha_{2}=0, \\
& \left(m_{1}^{2}-m_{2}^{2}+m_{3}^{2}\right) \alpha_{1}+\left(m_{1}^{2}-m_{2}^{2}-m_{3}^{2}\right) \alpha_{2}=0, \\
& 2 p \cdot q_{1} \alpha_{1}+\left(2 p \cdot q_{1}-s-m_{3}^{2}+m_{4}^{2}\right) \alpha_{2}=0, \\
& \left(m_{1}^{2}+m_{2}^{2}-m_{3}^{2}\right) \alpha_{2}-\left(m_{1}^{2}-m_{2}^{2}+m_{3}^{2}\right)\left(\alpha_{3}+\alpha_{5}\right)+\left(2 p \cdot q_{1}-m_{1}^{2}+m_{2}^{2}-m_{3}^{2}\right) \alpha_{4}=0, \\
& \left(m_{1}^{2}-m_{2}^{2}-m_{3}^{2}\right) \alpha_{2}-2 m_{3}^{2}\left(\alpha_{3}+\alpha_{5}\right)+\left(s-m_{3}^{2}-m_{4}^{2}\right) \alpha_{4}=0, \\
& \left(-2 p \cdot q_{1}+s+m_{3}^{2}-m_{4}^{2}\right) \alpha_{2}+\left(s+m_{3}^{2}-m_{4}^{2}\right)\left(\alpha_{3}+\alpha_{5}\right)-\left(s-m_{3}^{2}+m_{4}^{2}\right) \alpha_{4}=0 .
\end{aligned}
$$

Compatibility between the first three equations requires the conditions

$$
m_{3}^{2}=\left(m_{1}+m_{2}\right)^{2}, \quad p \cdot q_{1}=\frac{m_{1}^{2}}{m_{1}^{2}-m_{2}^{2}+m_{3}^{2}}\left(s+m_{3}^{2}-m_{4}^{2}\right) .
$$

As a consequence, it follows that

$$
\alpha_{1}=\frac{m_{2}}{m_{1}} \alpha_{2}, \quad \alpha_{2}=\frac{1}{m_{2}\left(m_{1}+m_{2}\right)}\left\{-\left(m_{1}+m_{2}\right)^{2}\left(\alpha_{3}+\alpha_{5}\right)+\frac{1}{2}\left[s-\left(m_{1}+m_{2}\right)^{2}-m_{4}^{2}\right] \alpha_{4}\right\},
$$

is a solution, if and only if $s=\left(m_{1}+m_{2} \pm m_{4}\right)^{2}$ and $m_{3}=m_{1}+m_{2}$, therefore, representing the leading Landau singularity. We are now in a position to attempt the evaluation of this topology. 


\subsection{Evaluation of $S^{131}$ for $m_{5}=m_{3}$}

In Eq. (167) we have a power $-2-\epsilon$ but for the first iteration we prefer to use the identity

$$
\left[1+\frac{1}{1+\epsilon}\left(y \partial_{y}+z \partial_{z}\right)\right] \chi_{131}^{-1-\epsilon}=\left(\mu_{x}^{2}-z^{2}\right) \chi_{131}^{-2-\epsilon}
$$

and obtain

$$
\begin{aligned}
S^{131} & =-\frac{1}{s}\left(\frac{\mu^{2}}{\pi s}\right)^{\epsilon} \Gamma(1+\epsilon) \int_{0}^{1} d x \int_{0}^{1} d y \int_{0}^{y} d z[x(1-x)]^{1+\epsilon / 2}(1-y)^{\epsilon / 2-1} \\
& \times(y-z) X_{131}^{-1-\epsilon}(x, y, z),
\end{aligned}
$$

with $X=x(1-x) \chi$. It is more convenient to change variables, $y=1-y^{\prime}$ and write

$$
\begin{aligned}
S^{131} & =-\frac{1}{s}\left(\frac{\mu^{2}}{\pi s}\right)^{\epsilon} \Gamma(1+\epsilon) \int_{0}^{1} d x \int_{0}^{1} d y \int_{0}^{1-y} d z[x(1-x)]^{1+\epsilon / 2} y^{\epsilon / 2-1} \\
& \times(1-y-z) X_{131}^{-1-\epsilon}(x, 1-y, z),
\end{aligned}
$$

where now the quadratic form is

$$
X_{131}=x(1-x) \chi_{131}, \quad \chi_{131}(x, 1-y, z)=z^{2}-\left(1+\mu_{3}^{2}-\mu_{4}^{2}\right) z+\left(\mu_{x}^{2}-\mu_{3}^{2}\right) y+\mu_{3}^{2},
$$

and where Eq.(26) can be used. We introduce

$$
b_{131}=\lambda\left(1, \mu_{3}^{2}, \mu_{4}^{2}\right)
$$

The finite part of $S^{131}$ becomes

$$
\begin{gathered}
S_{\mathrm{fin}}^{131}=-\frac{2}{s b_{131}} \sum_{n=0}^{3} I_{n}^{131} \\
I_{3}^{131}=\int_{0}^{1} d x \int_{0}^{1} d y \int_{0}^{1-y} d z\left[3 \ln X_{131}(x, y, z)+\left(2 z-1-\frac{1}{2} \mu_{34}^{2}\right)\left(\frac{\ln X_{131}(x, y, z)}{y}\right)_{+},\right. \\
I_{2}^{131}=\int_{0}^{1} d x \int_{0}^{1} d y\left\{\ln X_{131}(x, 0, y)\left[2(1-y)+\left(2 y-1-\frac{1}{2} \mu_{34}^{2}\right) L_{131}(x, y)\right]\right. \\
\left.+\frac{1}{2} \mu_{34}^{2}\left[\left(\frac{\ln X_{131}(x, y, 0)}{y}\right)_{+}-\ln X_{131}(x, y, 0)\right]\right\} \\
I_{1}^{131}=\frac{1}{2} \mu_{34}^{2} \int_{0}^{1} d x\left[\ln \left(x-x^{2}\right)-\ln X_{131}(x, 0,0)\right] \ln X_{131}(x, 0,0), \\
I_{0}^{131}=\frac{7}{2}
\end{gathered}
$$

where $L_{131}$ is given by

$$
L_{131}(x, y)=\ln (1-y)+\ln x+\ln (1-x)-\ln X_{131}(x, 0, y) .
$$


The obvious advantage of this result is that $b_{131}$ does not depend on $x$. However, $b_{131}=0$ for $s=\left(m_{3} \pm m_{4}\right)^{2}$. In this case we have a quadratic form $z^{2}+b z+c y+d$ where $4 d=b^{2}$. Therefore, we consider first the case $\mu_{34}^{2}=-2 \mu_{3}$; we have

$$
\chi_{131}(x, y, z)=\left(z+\mu_{3}\right)^{2}+\left(\mu_{x}^{2}-\mu_{3}^{2}\right) y .
$$

It is convenient to change variable, $z=z^{\prime}-\mu_{3}$, so that the integral to be evaluated becomes

$$
\begin{aligned}
J & =\int_{0}^{1} d y \int_{0}^{1-y} d z y^{\epsilon / 2-1}(1-y-z) \chi_{131}^{-1-\epsilon}(x, y, z) \\
& =\int_{0}^{1} d y \int_{\mu_{3}}^{1+\mu_{3}-y} d z y^{\epsilon / 2-1}\left(1+\mu_{3}-y-z\right)\left(z^{2}+c y\right)^{-1-\epsilon},
\end{aligned}
$$

with $c=\mu_{x}^{2}-\mu_{3}^{2}$. After changing variables, $z^{2}=t$ we use

$$
(t+c y)^{-1-\epsilon}=-\frac{1}{\epsilon} \partial_{t}(t+c y)^{-\epsilon},
$$

and integrate by parts. The final result, which is valid for $\sqrt{s}=m_{4}-m_{3}$ and $m_{4} \geq m_{3}$ can be cast into the following form:

$$
\begin{aligned}
J & =-\frac{1}{\epsilon}\left[\frac{\ln \mu_{3}^{2}}{\mu_{3}}-2\left(1+\mu_{3}\right) \int_{\mu_{3}}^{1+\mu_{3}} d y \frac{\ln y}{y^{2}}\right] \\
& -\frac{1}{2} \int_{0}^{1} d y \int_{\mu_{3}}^{1+\mu_{3}-y} \frac{d z}{z^{2}}\left[\ln \left(z^{2}+c y\right)-\left(1+\mu_{3}\right)\left(\frac{\ln \left(z^{2}+c y\right)}{y}\right)_{+}\right] \\
& +\left(1+\mu_{3}\right) \int_{\mu_{3}}^{1+\mu_{3}} d y\left[\frac{\ln y \ln \left(1+\mu_{3}-y\right)}{y^{2}}-\frac{\ln ^{2} y}{y^{2}}\right] \\
& +\frac{1}{2 \mu_{3}} \int_{0}^{1} d y\left[\ln \left(\mu_{3}^{2}+c y\right)-\left(\frac{\ln \left(\mu_{3}^{2}+c y\right)}{y}\right)_{+}\right]+\frac{1}{2 \mu_{3}} \ln ^{2} \mu_{3}^{2} .
\end{aligned}
$$

The case $\sqrt{s}=m_{3}-m_{4}$, with $m_{3} \geq m_{4}$ is obtained after the substitution $z=1-z^{\prime}$ in Eq.(186). We obtain

$$
\begin{aligned}
J & =\int_{0}^{1} d y \int_{y}^{1} d z y^{\epsilon / 2-1}(z-y) \chi_{131}^{-1-\epsilon}(x, y, 1-z) \\
& =\int_{0}^{1} d y \int_{y+\mu_{4}}^{1+\mu_{4}} d z y^{\epsilon / 2-1}\left(z-\mu_{4}-y\right)\left(z^{2}+c y\right)^{-1-\epsilon}, \\
\chi_{131}(x, y, 1-z) & =z^{2}-\mu_{43}^{2} z+\left(\mu_{x}^{2}-\mu_{3}^{2}\right) y+\mu_{4}^{2},
\end{aligned}
$$

and the case considered here corresponds to $\mu_{43}^{2}=-2 \mu_{4}$. Therefore the pseudo-threshold $s=$ $\left(m_{3}-m_{4}\right)^{2}$ is covered.

Always for $m_{5}=m_{3}$ we have a second method which is based on the observation that the following identity holds:

$$
S^{131}=-\int_{0}^{1} d x(1-x) \frac{\partial}{\partial M_{x}^{2}}(\epsilon-1) S_{33}\left(x^{2} p^{2} ; m_{1}, m_{2}, M_{x}\right),
$$


with $\mu_{x}^{2}=M_{x}^{2} / s$ and $\mu_{x}^{2}=x^{2}-\mu_{34}^{2} x+\mu_{3}^{2}$. The idea is to change variable $x \rightarrow \mu_{x}^{2}$ in the integral and perform integration by parts. Unless numerical differentiation is used, this method will fail at $b_{131}=0$ and, therefore, it will be no longer discussed.

\subsubsection{Evaluation around $s=\left(m_{3}+m_{4}\right)^{2}$}

In this region we use

$$
\mu_{3}^{2}=\frac{1}{4}\left(\mu_{34}^{4}-\lambda\right), \quad \lambda \equiv \lambda\left(1, \mu_{3}^{2}, \mu_{4}^{2}\right),
$$

and write the master integral $J$ of Eq.(186) as

$$
J=\int_{0}^{1} d y \int_{-z_{m}}^{1-z_{m}-y} d z y^{\epsilon / 2-1}\left(1-z_{m}-y-z\right)\left(z^{2}+c y-\frac{1}{4} \lambda\right)^{-1-\epsilon},
$$

where $z_{m}=\mu_{34}^{2} / 2$ and $c=\mu_{x}^{2}-\mu_{3}^{2}$. As a first step we will set $\lambda=0$ whenever possible; $J$ is the sum of three contributions, the first one being

$$
J_{1}=-\int_{0}^{1} d y \int_{-z_{m}}^{1-z_{m}-y} d z y^{\epsilon / 2-1} z\left(z^{2}+c y\right)^{-1-\epsilon} .
$$

Here we use the identity

$$
-\frac{1}{\epsilon} \partial_{z}\left(z^{2}+c y\right)^{-\epsilon}=2 z\left(z^{2}+c y\right)^{-1-\epsilon},
$$

which gives, after integration by parts, the following result:

$$
\begin{aligned}
J_{1} & =\frac{2}{\epsilon} \ln \frac{z_{m}}{1-z_{m}}+2\left[\ln ^{2} z_{m}-\ln ^{2}\left(1-z_{m}\right)\right] \\
& +\frac{1}{2} \int_{0}^{1} d y\left[\frac{\ln \eta\left(y,-z_{m}\right)}{y}-\frac{\ln \eta\left(y, 1-z_{m}-y\right)}{y}\right]_{+},
\end{aligned}
$$

where $\eta(y, z)=z^{2}+c y$. The second contribution, $J_{2}$ is defined by

$$
J_{2}=-\int_{0}^{1} d y \int_{-z_{m}}^{1-z_{m}-y} d z y^{\epsilon / 2}\left(z^{2}+c y\right)^{-1-\epsilon} .
$$

Here we will us another identity, i.e.

$$
-\frac{1}{\epsilon} \partial_{y}\left(z^{2}+c y\right)^{-\epsilon}=c\left(z^{2}+c y\right)^{-1-\epsilon},
$$

giving, after integration by parts, the following result:

$$
J_{2}=-\frac{1}{c} \int_{-z_{m}}^{1-z_{m}} d z\left[\ln \eta\left(1-z_{m}-z, z\right)-\ln \eta(0, z)\right] .
$$


The last contribution is the most difficult to obtain. We start from the definition of $J_{3}$,

$$
J_{3}=-\int_{0}^{1} d y \int_{-z_{m}}^{1-z_{m}-y} d z y^{\epsilon / 2-1}\left(z^{2}+c y\right)^{-1-\epsilon} .
$$

The $y$ integration range is further divided into two regions, $\left[0,1-z_{m}\right]$ and $\left[1-z_{m}, 1\right]$. In the former one $1-z_{m}-y$ is positive and we write

$$
J_{31}=\int_{0}^{1-z_{m}} d y y^{\epsilon / 2-1} \sum_{i=1}^{2} \int_{0}^{Y_{i}} d z\left(z^{2}+c y\right)^{-1-\epsilon},
$$

where $Y_{1}=z_{m}$ and $Y_{2}=1-z_{m}-y$. Therefore $J_{31}$ is a combination of new integrals denoted by

$$
H_{i}=\int_{0}^{Y_{i}} d z\left(z^{2}+c y\right)^{-1-\epsilon}=(c y)^{-1-\epsilon} Y_{i 2} F_{1}\left(1+\epsilon, \frac{1}{2} ; \frac{3}{2} ;-\frac{Y_{i}^{2}}{c y}\right) .
$$

Using a well known property of the hypergeometric functions [16] we obtain

$$
\begin{aligned}
& { }_{2} F_{1}\left(1+\epsilon, \frac{1}{2} ; \frac{3}{2} ;-\frac{Y_{i}^{2}}{c y}\right)=B_{2}\left(\frac{c}{Y_{i}^{2}} y\right)^{1 / 2} \\
+ & B_{1}\left(\frac{c}{Y_{i}^{2}} y\right)^{1+\epsilon}{ }_{2} F_{1}\left(1+\epsilon, \frac{1}{2}+\epsilon ; \frac{3}{2}+\epsilon ;-\frac{c}{Y_{i}^{2}} y\right),
\end{aligned}
$$

where the coefficients $B_{1,2}$ are as follows:

$$
\begin{aligned}
& B_{1}=\frac{\Gamma(3 / 2) \Gamma(-1 / 2-\epsilon)}{\Gamma(1 / 2) \Gamma(1 / 2-\epsilon)}=-\frac{1}{1+2 \epsilon}, \\
& B_{2}=\frac{\Gamma(3 / 2) \Gamma(1 / 2+\epsilon)}{\Gamma(1+\epsilon)}=\frac{1}{2} \pi\left\{1+\epsilon\left[\gamma+\psi\left(\frac{1}{2}\right)\right]+\mathcal{O}\left(\epsilon^{2}\right)\right\},
\end{aligned}
$$

where $\psi(z)$ is the Euler psi-function [16. In this way the functions $H_{i}$ become

$$
H_{i}=B_{1} Y_{i}^{-1-2 \epsilon}{ }_{2} F_{1}\left(1+\epsilon, \frac{1}{2}+\epsilon ; \frac{3}{2}+\epsilon ;-\frac{c}{Y_{i}^{2}} y\right)+B_{2}(c y)^{-1 / 2-\epsilon} .
$$

At this point we observe that the term proportional to $B_{2}$ will give rise to a divergency after the $y$-integration. If $\lambda$ is kept different from zero then Eq.(204) becomes

$$
H_{i}=B_{1} Y_{i}^{-1-2 \epsilon}{ }_{2} F_{1}\left(1+\epsilon, \frac{1}{2}+\epsilon ; \frac{3}{2}+\epsilon ;-\frac{c y-\lambda / 4}{Y_{i}^{2}}\right)+B_{2}\left(c y-\frac{1}{4} \lambda\right)^{-1 / 2-\epsilon} .
$$

Consider now the $y$-integration for the second term: we obtain

$$
H_{i 2}=\int_{0}^{1-z_{m}} d y y^{\epsilon / 2-1}\left(c y-\frac{1}{4} \lambda\right)^{-1 / 2-\epsilon}
$$




$$
\begin{aligned}
& =2\left(-\frac{1}{4} \lambda\right)^{-1 / 2-\epsilon} \frac{\left(1-z_{m}\right)^{\epsilon / 2}}{\epsilon}{ }_{2} F_{1}\left(\frac{1}{2}+\epsilon, \frac{\epsilon}{2} ; 1+\frac{\epsilon}{2} ; 4 \frac{c}{\lambda}\left(1-z_{m}\right)\right) \\
& =2 \frac{b_{1}}{\epsilon}\left(1-z_{m}\right)^{-1 / 2-\epsilon / 2} c^{-1 / 2-\epsilon}{ }_{2} F_{1}\left(\frac{1}{2}+\epsilon, \frac{1+\epsilon}{2} ; \frac{3+\epsilon}{2} ; \frac{\lambda}{4 c\left(1-z_{m}\right)}\right) \\
& +2 \frac{b_{2}}{\epsilon} c^{-\epsilon / 2}\left(-\frac{1}{4} \lambda\right)^{-1 / 2-\epsilon / 2},
\end{aligned}
$$

where the coefficients $b_{1,2}$ are

$$
b_{1}=\frac{\Gamma(-1 / 2-\epsilon / 2)}{\Gamma(1 / 2-\epsilon / 2)} \frac{\epsilon}{2}, \quad b_{2}=\frac{\Gamma(1+\epsilon / 2) \Gamma(1 / 2+\epsilon / 2)}{\Gamma(1 / 2+\epsilon)} .
$$

For the term proportional to $b_{1}$ there is no UV pole and we may set $\epsilon=0$. Therefore we get

$$
\begin{aligned}
H_{i 2} & =-2\left(1-z_{m}\right)^{-1 / 2} c_{2}^{-1 / 2} F_{1}\left(\frac{1}{2}, \frac{1}{2} ; \frac{3}{2} ; \frac{\lambda}{4 c\left(1-z_{m}\right)}\right)+2 \frac{b_{2}}{\epsilon} c^{-\epsilon / 2}\left(-\frac{1}{4} \lambda\right)^{-1 / 2-\epsilon / 2} \\
& =-4 \lambda^{-1 / 2} \arcsin \left[\frac{\lambda}{4 c\left(1-z_{m}\right)}\right]^{1 / 2}+2(-\lambda)^{-1 / 2}\left[\frac{2}{\epsilon}-\ln c-\ln \left(-\frac{\lambda}{4}\right)-\gamma-\psi\left(\frac{1}{2}\right)\right] .
\end{aligned}
$$

Setting $\lambda=0$ whenever possible produces the following result:

$$
H_{i 2}=-2\left(1-z_{m}\right)^{-1 / 2} c^{-1 / 2}+2(-\lambda-i \delta)^{-1 / 2}\left[\frac{2}{\epsilon}-\ln c-\ln \left(-\frac{\lambda}{4}-i \delta\right)-\gamma-\psi\left(\frac{1}{2}\right)\right]
$$

where $\delta \rightarrow 0_{+}$. Returning to Eq. (205) we consider the part proportional to $B_{1}$ and the corresponding $y$-integral which becomes

$$
H_{i 1}=\int_{0}^{1-z_{m}} d y y^{\epsilon / 2-1} f_{i}(\epsilon, y) \quad f_{i}(\epsilon, y)=Y_{i}^{-1-2 \epsilon}{ }_{2} F_{1}\left(1+\epsilon, \frac{1}{2}+\epsilon ; \frac{3}{2}+\epsilon ;-\frac{c}{Y_{i}^{2}} y\right) .
$$

We split the integral into two parts, according to the following recipe:

$$
\begin{aligned}
H_{i 1} & =\int_{0}^{1-z_{m}} d y\left\{y^{\epsilon / 2-1} f_{i}(\epsilon, 0)+y^{\epsilon / 2}\left[\frac{f_{i}(\epsilon, y)}{y}\right]_{+}\right\} \\
& =\frac{2}{\epsilon}\left(1-z_{m}\right)^{\epsilon / 2} f_{i}(\epsilon, 0)+\int_{0}^{1-z_{m}} d y\left[\frac{f_{i}(0, y)}{y}\right]_{+}+\mathcal{O}(\epsilon) .
\end{aligned}
$$

The function $f_{i}$ is defined by

$$
\begin{aligned}
& f_{i}(\epsilon, 0)=Y_{i}^{-1-2 \epsilon}(y=0), \quad f_{i}(0,0)=\frac{1}{Y_{i}(y=0)} \\
& f_{i}(0, y)=\frac{1}{Y_{i}}{ }_{2} F_{1}\left(1, \frac{1}{2} ; \frac{3}{2} ;-\frac{c y}{Y_{i}^{2}}\right)=\frac{1}{2}(-c y)^{-1 / 2} \ln \frac{Y_{i}+(-c y)^{1 / 2}}{Y_{i}-(-c y)^{1 / 2}} .
\end{aligned}
$$


Since $J_{31}$ is the following combination,

$$
J_{31}=\sum_{i=1}^{2}\left(B_{1} H_{i 1}+B_{2} H_{i 2}\right)
$$

we obtain the final result by further inserting

$$
\begin{aligned}
& 2 \frac{B_{1}}{\epsilon} Y_{1}^{-1-2 \epsilon}(y=0)\left(1-z_{m}\right)^{\epsilon / 2}=-\frac{2}{z_{m}}\left[\frac{1}{\epsilon}+\frac{1}{2} \ln \left(1-z_{m}\right)-2 \ln z_{m}-2\right], \\
& 2 \frac{B_{1}}{\epsilon} Y_{2}^{-1-2 \epsilon}(y=0)\left(1-z_{m}\right)^{\epsilon / 2}=-\frac{2}{1-z_{m}}\left[\frac{1}{\epsilon}-\frac{3}{2} \ln \left(1-z_{m}\right)-2\right] .
\end{aligned}
$$

The second part of the function is $J_{32}$ defined by

$$
J_{32}=\int_{1-z_{m}}^{1} d y y^{\epsilon / 2-1} \int_{-Y_{2}}^{Y_{1}} d z\left(z^{2}+c y\right)^{-1-\epsilon}
$$

where we can set $\epsilon=0$ from the beginning. We obtain

$$
\begin{aligned}
J_{32} & =-\frac{1}{c} \int_{1-z_{m}}^{1} \frac{d y}{y^{2}} \sum_{i=1}^{2}(-1)^{i}\left|Y_{i}\right|{ }_{2} F_{1}\left(1, \frac{1}{2} ; \frac{3}{2} ;-\frac{Y_{i}^{2}}{c y}\right) \\
& =\frac{1}{2} \int_{1-z_{m}}^{1} \frac{d y}{y} \sum_{i=1}^{2}(-1)^{i}(-c y)^{-1 / 2} \ln \frac{(-c y)^{1 / 2}+\left|Y_{i}\right|}{(-c y)^{1 / 2}-\left|Y_{i}\right|}
\end{aligned}
$$

In all formulas the square roots must be understood with the replacement $c y \rightarrow c y-i \delta$, where $\delta \rightarrow 0_{+}$.

Let us return to Eq.(204) and consider also the term proportional to $B_{1}$ for $\lambda \neq 0$. In this case we will use again Eq.(211) but with

$$
f_{i}(\epsilon, y)=Y_{i}^{-1-2 \epsilon}{ }_{2} F_{1}\left(1+\epsilon, \frac{1}{2}+\epsilon ; \frac{3}{2}+\epsilon ;-\frac{c y-\lambda / 4}{Y_{i}^{2}}\right) .
$$

Due to the pole at $\epsilon=0$ we must expand $f_{i}(\epsilon, 0)$ to $\mathcal{O}(\epsilon)$. We derive

$$
{ }_{2} F_{1}\left(1+\epsilon, \frac{1}{2}+\epsilon ; \frac{3}{2}+\epsilon ; y_{i}^{2}\right)=\frac{\Gamma(3 / 2+\epsilon)}{\Gamma(1 / 2+\epsilon)} \int_{0}^{1} d t t^{-1 / 2}\left(1-y_{i}^{2} t\right)^{-1}\left\{1+\epsilon\left[\ln t-\ln \left(1-y_{i}^{2} t\right)\right]\right\},
$$

where we have introduced $y_{i}^{2}=\lambda /\left(4 Y_{i}^{2}\right)$. Therefore, expanding around $\epsilon=0$, we obtain

$$
\begin{aligned}
& { }_{2} F_{1}\left(1+\epsilon, \frac{1}{2}+\epsilon ; \frac{3}{2}+\epsilon ; y_{i}^{2}\right)=\frac{1}{2 x_{i}} \ln \frac{1+x_{i}}{1-x_{i}}+\epsilon \mathcal{F}, \\
& \mathcal{F}=-\int_{0}^{1} d x \frac{\left[2 \ln x-\ln \left(1-y_{i}^{2} x^{2}\right)\right]}{y_{i}^{2} x^{2}-1}+\frac{1}{x_{i}} \ln \frac{1+x_{i}}{1-x_{i}},
\end{aligned}
$$


a result which is based on the standard integral representation of the hypergeometric function [16]. The integral can be computed exactly; introducing $x_{i}=\sqrt{y_{i}^{2}}=\sqrt{\lambda} /\left(2 Y_{i}\right)$ we obtain

$$
\begin{aligned}
\mathcal{F} & =-\frac{1}{x_{i}}\left[\operatorname{Li}_{2}\left(x_{i}\right)-\mathrm{Li}_{2}\left(-x_{i}\right)\right]-\frac{1}{4 x_{i}}\left[\ln ^{2}\left(1+x_{i}\right)-\ln ^{2}\left(1-x_{i}\right)\right] \\
& -\frac{1}{2 x_{i}}\left[\ln 2 \ln \left(1+x_{i}\right)+\operatorname{Li}_{2}\left(\frac{1+x_{i}}{2}\right)-\operatorname{Li}_{2}\left(\frac{1}{2}\right)\right] \\
& +\frac{1}{2 x_{i}}\left[\ln 2 \ln \left(1-x_{i}\right)+\operatorname{Li}_{2}\left(\frac{1-x_{i}}{2}\right)-\operatorname{Li}_{2}\left(\frac{1}{2}\right)\right]+\frac{1}{x_{i}} \ln \frac{1+x_{i}}{1-x_{i}}
\end{aligned}
$$

where $L i_{2}(1 / 2)=\left(\zeta(2)-\ln ^{2} 2\right) / 2$. To obtain Eq.(220) we have used the following integrals 円:

$$
\begin{aligned}
& \int_{0}^{1} d x \frac{\ln x}{x \pm x_{i}^{-1}}=\operatorname{Li}_{2}\left(\mp x_{i}\right), \quad \int_{0}^{1} d x \frac{\ln \left(1 \pm x_{i} x\right)}{1 \pm x_{i} x}= \pm \frac{1}{2 x_{i}} \ln ^{2}\left(1 \pm x_{i}\right), \\
& \int_{0}^{1} d x \frac{\ln \left(1 \pm x_{i} x\right)}{1 \mp x_{i} x}=\mp \frac{1}{x_{i}}\left[\ln 2 \ln \left(1 \mp x_{i}\right)-\operatorname{Li}_{2}\left(\frac{1 \mp x_{i}}{2}\right)+\operatorname{Li}_{2}\left(\frac{1}{2}\right)\right] .
\end{aligned}
$$

Furthermore, $\operatorname{Li}_{2}(1 / 2)=\left(\zeta(2)-\ln ^{2} 2\right) / 2$. The implementation of this result requires distorting the $x$-integration contour for $J_{2}$ (Eq.(198)) in order to avoid the points

$$
x_{ \pm}=\frac{1}{2 \mu_{3}^{2}}\left[\mu_{1}^{2}-\mu_{2}^{2}+\mu_{3}^{2} \pm \lambda^{1 / 2}\left(\mu_{3}^{2}, \mu_{1}^{2}, \mu_{2}^{2}\right)\right] \text {. }
$$

These two points coincide for $\mu_{3}^{2}=\left(\mu_{1}+\mu_{2}\right)^{2}$. In practical cases this may happens for $m_{1}=m_{3}$ and $m_{2}=0$ where we have

$$
\mu_{x}^{2}=\frac{\mu_{1}^{2}}{x}, \quad c=\mu_{x}^{2}-\mu_{3}^{2}=\mu_{1}^{2} \frac{1-x}{x}
$$

Actually there is no problem at $x=1(c=0)$ since the result for $J_{2}$ is

$$
J_{2}=\frac{1}{\mu_{1}^{2}} \frac{x}{1-x} \int_{-z_{m}}^{1-z_{m}} d z\left\{\ln z^{2}-\ln \left[z^{2}+\mu_{1}^{2} \frac{1-x}{x}\left(1-z_{m}-z\right)\right]\right\} .
$$

Our result for the threshold region deviates from the main approach of expressing the integrand as products of polynomials and logarithms of polynomials and requires some analytical work before attempting the numerical evaluation. However, in this way, we have been able to isolate the square root singularity which shows up in the coefficient of the single pole and will disappear after including the corresponding subtraction diagram. The net result can be safely computed by numerical integration.

\footnotetext{
${ }^{1}$ We disagree with Eq.(1) p. 34 of ref. [30]
} 


\subsection{Derivative of $S^{131}$ and infrared poles}

Self-energy diagrams enter into the calculation of wave function renormalization factor and, therefore, also their derivative with respect to $p^{2}$ is needed. We concentrate on the on-shell derivative of $S^{131}$ where $m_{5}=m_{3}$ and compute

$$
\begin{aligned}
& S_{p}^{131} \equiv \frac{\partial}{\partial p^{2}} S^{131}\left(p^{2} ; m_{1}, m_{2}, m_{3}, m_{4}, m_{3}\right)= \\
- & \frac{1}{2 p^{2}}\left[\left(p^{2}+m_{3}^{2}-m_{4}^{2}\right) S^{141}\left(p^{2} ; m_{1}, m_{2}, m_{3}, m_{4}, m_{3}, m_{4}\right)\right. \\
+ & \left.S^{131}\left(p^{2} ; m_{1}, m_{2}, m_{3}, m_{4}, m_{3}\right)-S^{131}\left(p^{2} ; m_{1}, m_{2}, m_{3}, m_{4}, m_{4}\right)\right] .
\end{aligned}
$$

The result for the derivative is

$$
\begin{aligned}
S_{p}^{131} & =-\frac{1}{s^{2}}\left(\frac{\mu^{2}}{\pi s}\right)^{\epsilon} \frac{1}{\epsilon} \int_{0}^{1} d x[x(1-x)]^{-\epsilon / 2} \int_{0}^{1} d y \int_{0}^{y} d z(1-y)^{\epsilon / 2} \\
& \times\left\{\Gamma(3+\epsilon) z(y-z)\left(\mu_{3}^{2}-\mu_{4}^{2}-1\right)\left[\left(\mu_{x}^{2}-z^{2}\right) \chi_{131}^{-3-\epsilon}+\frac{2-\epsilon / 2}{2+\epsilon} \chi_{131}^{-2-\epsilon}\right]\right. \\
& \left.+\Gamma(2+\epsilon)(y-2 z)\left[\left(\mu_{x}^{2}-z^{2}\right) \chi_{131}^{-2-\epsilon}+\frac{2-\epsilon / 2}{1+\epsilon} \chi_{131}^{-1-\epsilon}\right]\right\} .
\end{aligned}
$$

It is important to recall once more that a necessary condition for the presence of infrared divergences is that the Landau equations are fulfilled. In the case of $S_{p}^{131}$ we see that $s=\left(m_{1}+m_{2} \pm m_{4}\right)^{2}$ and $m_{3}^{2}=\left(m_{1}+m_{2}\right)^{2}$ are satisfied by the QED-like configuration, $m_{2}=m_{4}=0, m_{1}=m_{3}=m$ and $s=m^{2}$ but, for instance, they are not when $m_{1}=m_{3}=m, m_{2}=0$ and $s=m^{2}$ but $m_{4}=M$. This configuration, that corresponds to electron (or $W$-boson) self-energy with $2 \equiv \gamma$ and $4 \equiv Z$, is therefore free from infrared poles.

Let us consider a realistic case, Fig. 10 which represents one of the contributions to the electron self-energy in QED. In this case the configuration is

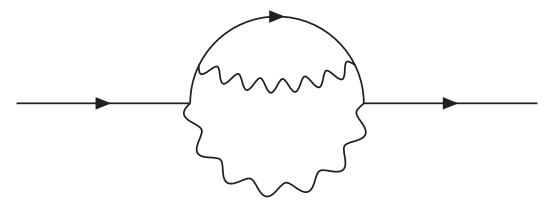

Figure 10: An $S^{131}$-type electron self-energy in QED.

a) $\quad m_{1}=m_{3}=m$ and $m_{2}=m_{4}=0$

and, therefore

$$
\chi_{131}=z^{2}-\left(1+\mu^{2}\right) z+\left(\mu^{2}-\mu_{x}^{2}\right) y+\mu_{x}^{2}, \quad \text { with } \quad \mu_{x}^{2}=\frac{\mu^{2}}{x}
$$


with $\mu^{2}=m^{2} / s$. The derivative is needed for on-shell electrons, therefore $\mu^{2}=1$ and

$$
\chi_{\mathrm{os} ; 131}=z^{2}-2 z+\left(1-\frac{1}{x}\right) y+\frac{1}{x} .
$$

We therefore obtain

$$
\begin{aligned}
\left.S_{p}^{131}\right|_{\mathrm{os}} & =-\frac{1}{m^{4}}\left(\frac{\mu^{2}}{\pi m^{2}}\right)^{\epsilon} \frac{\Gamma(2+\epsilon)}{\epsilon} \int_{0}^{1} d x[x(1-x)]^{-\epsilon / 2} \int_{0}^{1} d y \int_{0}^{y} d z(1-y)^{\epsilon / 2} \\
& \times(y-2 z)\left[\left(\frac{1}{x}-z^{2}\right) \chi_{\mathrm{os} ; 131}^{-2-\epsilon}+\frac{2-\epsilon / 2}{1+\epsilon} \chi_{\mathrm{os} ; 131}^{-1-\epsilon}\right] .
\end{aligned}
$$

The well-known infrared singularity shows up in parametric space at $y=z=1$. Note that the diagram itself is not infrared divergent but only the on-shell derivative, due to the factor $y-z$ in the integral of Eq.(167). To continue our derivation, we use the identity

$$
\left[1+\frac{1}{1+\epsilon}\left(y \partial_{y}+z \partial_{z}\right)\right] \chi_{\mathrm{os} ; 131}^{-1-\epsilon}=\left(\frac{1}{x}-z^{2}\right) \chi_{\mathrm{os} ; 131}^{-2-\epsilon}
$$

to write the on-shell derivative as

$$
\begin{aligned}
\left.S_{p}^{131}\right|_{\mathrm{os}} & =-\frac{1}{m^{4}}\left(\frac{\mu^{2}}{\pi m^{2}}\right)^{\epsilon} \Gamma(\epsilon) \int_{0}^{1} d x[x(1-x)]^{-\epsilon / 2} \int_{0}^{1} d y \int_{0}^{y} d z(1-y)^{\epsilon / 2} \\
& \times(y-2 z)\left[3+\frac{\epsilon}{2}+\left(y \partial_{y}+z \partial_{z}\right)\right] \chi_{\mathrm{os} ; 131}^{-1-\epsilon} .
\end{aligned}
$$

After integration by parts we obtain

$$
\begin{aligned}
\left.S_{p}^{131}\right|_{\mathrm{os}} & =-\frac{1}{2 m^{4}}\left(\frac{\mu^{2}}{\pi m^{2}}\right)^{\epsilon} \Gamma(1+\epsilon) \int_{0}^{1} d x[x(1-x)]^{-\epsilon / 2} \\
& \times \int_{0}^{1} d y \int_{0}^{y} d z(y-2 z)(1-y)^{\epsilon / 2-1} \chi_{\mathrm{os} ; 131}^{-1-\epsilon} \\
& =-\frac{1}{2 m^{4}}\left(\frac{\mu^{2}}{\pi m^{2}}\right)^{\epsilon} \Gamma(1+\epsilon) \int_{0}^{1} d x[x(1-x)]^{-\epsilon / 2} \\
& \times \int_{0}^{1} d y \int_{y}^{1} d z(2 z-y-1) y^{\epsilon / 2-1}\left[z^{2}+\left(\frac{1}{x}-1\right) y\right]^{-1-\epsilon} .
\end{aligned}
$$

The total result is the sum of three contributions. We write

$$
J=\int_{0}^{1} d y \int_{y}^{1} d z(2 z-y-1) y^{\epsilon / 2-1}\left[z^{2}+\left(\frac{1}{x}-1\right) y\right]^{-1-\epsilon}=J_{1}-J_{2}-J_{3},
$$

and introduce $X=1 / x-1$. For $J_{1}$ we obtain

$$
\begin{aligned}
J_{1} & =-\frac{1}{\epsilon} \int_{0}^{1} d y \int_{y}^{1} d z y^{\epsilon / 2-1} \partial_{z}\left(z^{2}+X y\right)^{-\epsilon} \\
& =-\frac{1}{\epsilon} \int_{0}^{1} d y\left[y^{\epsilon / 2-1}(1+X y)^{-\epsilon}-y^{-\epsilon / 2-1}(y+X)^{-\epsilon}\right] .
\end{aligned}
$$


For computing the remaining two integrals we use the following relation

$$
\left[1+\frac{1}{1+\epsilon}\left(\frac{1}{2} z \partial_{z}+y \partial_{y}\right)\right]\left(z^{2}+X y\right)^{-1-\epsilon}=0 .
$$

After integration by parts we obtain the following results:

$$
\begin{aligned}
& (1-\epsilon) J_{2}=\int_{0}^{1} d y\left[y^{\epsilon / 2}(1+X y)^{-1-\epsilon}+y^{-\epsilon / 2}(y+X)^{-1-\epsilon}\right], \\
& (1+\epsilon) J_{3}=-\int_{0}^{1} d y\left[y^{\epsilon / 2-1}(1+X y)^{-1-\epsilon}+y^{-\epsilon / 2-1}(y+X)^{-1-\epsilon}\right] .
\end{aligned}
$$

The $J_{i}$ integrals are successively folded to become

$$
I_{i}=\int_{0}^{1} d x[x(1-x)]^{-\epsilon / 2} J_{i}
$$

We illustrate in details one particular case and refer to Appendix C for further details:

$$
\mathcal{J}=\int_{0}^{1} d y y^{-\epsilon / 2-1}(y+X)^{-\epsilon-1} .
$$

This integral gives rise to an hypergeometric function [16],

$$
\mathcal{J}=-\frac{2}{\epsilon} X^{-1-\epsilon}{ }_{2} F_{1}\left(1+\epsilon,-\frac{\epsilon}{2} ; 1-\frac{\epsilon}{2} ;-\frac{1}{X}\right) .
$$

Using well-known properties of the hypergeometric functions [16] we obtain

$$
\begin{aligned}
\mathcal{J} & =-\frac{2}{\epsilon} x^{1+\epsilon}{ }_{2} F_{1}\left(1+\epsilon, 1 ; 1-\frac{\epsilon}{2} ; x\right) \\
& =-\frac{2}{\epsilon} x^{1+\epsilon}\left[A_{1}{ }_{2} F_{1}\left(1+\epsilon, 1 ; 2+\frac{3}{2} \epsilon ; 1-x\right)\right. \\
& \left.+A_{2}(1-x)^{-1-3 / 2 \epsilon}{ }_{2} F_{1}\left(-\frac{3}{2} \epsilon,-\frac{\epsilon}{2} ;-\frac{3}{2} \epsilon ; 1-x\right)\right] \\
& =-\frac{2}{\epsilon} x^{1+\epsilon}\left[A_{1}{ }_{2} F_{1}\left(1+\epsilon, 1 ; 2+\frac{3}{2} \epsilon ; 1-x\right)+A_{2} x^{\epsilon / 2}(1-x)^{-1-3 / 2 \epsilon}\right],
\end{aligned}
$$

where the coefficients $A_{i}$ are given by

$$
A_{1}=\frac{\Gamma(1-\epsilon / 2) \Gamma(-1-3 / 2 \epsilon)}{\Gamma(-3 / 2 \epsilon) \Gamma(-\epsilon / 2)}=\frac{\epsilon}{2+3 \epsilon}, \quad A_{2}=\frac{\Gamma(1-\epsilon / 2) \Gamma(1+3 / 2 \epsilon)}{\Gamma(1+\epsilon)} .
$$

We next compute

$$
\mathcal{I}=\int_{0}^{1} d x[x(1-x)]^{-\epsilon / 2} \mathcal{J}=\mathcal{I}_{1}+\mathcal{I}_{2}
$$


The first integral is

$$
\begin{aligned}
\mathcal{I}_{1} & =-\frac{2}{\epsilon} \frac{\Gamma(1-\epsilon / 2) \Gamma(1+3 / 2 \epsilon)}{\Gamma(1+\epsilon)} \int_{0}^{1} d x x^{1+\epsilon}(1-x)^{-1-2 \epsilon} \\
& =-\frac{2}{\epsilon} \frac{\Gamma(1-\epsilon / 2) \Gamma(1+3 / 2 \epsilon) \Gamma(2+\epsilon) \Gamma(-2 \epsilon)}{\Gamma(1+\epsilon) \Gamma(2-\epsilon)}=-\frac{2}{\epsilon} \Gamma(-2 \epsilon)+\mathcal{R} .
\end{aligned}
$$

In expanding around $\epsilon=0$ we use

$$
\begin{aligned}
\Gamma(-2 \epsilon) & =-\frac{1}{2 \epsilon}-\gamma-\left[\gamma^{2}+\zeta(2)\right] \epsilon+\mathcal{O}\left(\epsilon^{2}\right) \\
\Gamma(a+\lambda) & =\Gamma(a)\left\{1+\psi(a) \lambda+\frac{1}{2} \lambda^{2}\left[\psi^{2}(a)+\psi^{\prime}(a)\right]+\mathcal{O}\left(\lambda^{3}\right)\right\} .
\end{aligned}
$$

It follows that

$$
\mathcal{I}_{1}=\frac{1}{\epsilon^{2}}+\frac{2}{\epsilon}+2+\frac{11}{4} \zeta(2)
$$

The second integral will be

$$
\mathcal{I}_{2}=-\frac{2}{2+3 \epsilon} \sum_{n=0}^{\infty} \frac{\Gamma(2+3 / 2 \epsilon)}{\Gamma(1+\epsilon)} \frac{\Gamma(n+1+\epsilon)}{\Gamma(n+2+3 / 2 \epsilon)} \int_{0}^{1} d x x^{1+\epsilon / 2}(1-x)^{n-\epsilon / 2},
$$

where we have introduced the hypergeometric series. After integrating over $x$ we can put $\epsilon=0$ and obtain that

$$
\mathcal{I}_{2}=1-\zeta(2)
$$

We also have the subtraction diagram of Fig. 9. The counter-term, in the MS-scheme, is $-2 i \pi^{2} / \epsilon$. After taking the derivative with respect to $p^{2}$ we put $p^{2}=-m^{2}$ and obtain

$$
\left.S_{\bar{p}}^{131}\right|_{\text {os }}=-\frac{2}{m^{4}}\left(\frac{\mu^{2}}{\pi m^{2}}\right)^{\epsilon} \frac{\Gamma(2+\epsilon / 2)}{\epsilon} \int_{0}^{1} d x x(1-x)^{-2-\epsilon} .
$$

In both cases one has to approach the mass-shell in a interval of the dimension $n$ where the integral is convergent and continue the result in the number of space-time dimensions. For instance, from Eq.(250) we obtain

$$
\left.S_{\bar{p}}^{131}\right|_{\mathrm{os}}=-\frac{1}{m^{4}}\left(\frac{\mu^{2}}{\pi m^{2}}\right)^{\epsilon}\left[\frac{2}{\epsilon^{2}}-(1+\gamma) \frac{1}{\epsilon}+1+\frac{1}{2} \gamma+\frac{1}{4} \gamma^{2}+\frac{1}{4} \zeta(2)+\mathcal{O}(\epsilon)\right] .
$$

Consider now another configuration, depicted in Fig. 11,

$$
\text { b) } \quad m_{1}=m_{2}=m, \quad m_{3}=m_{5}=0 \quad \text { and } \quad m_{4}=M \text {. }
$$

It is immediately seen that b) does not correspond to the leading Landau singularity but rather 


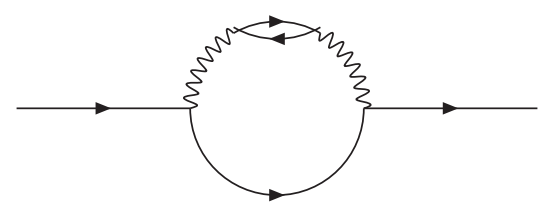

Figure 11: A second $S^{131}$-type electron self-energy in QED. The innermost loop can be a fermion of any flavor.

to the sub-leading one corresponding to $\alpha_{1}=\alpha_{2}=0$ and $s=M^{2}$. We start from

$$
S^{131}=-\left(\frac{\mu^{2}}{\pi}\right)^{\epsilon} \Gamma(1+\epsilon) \int_{0}^{1} d x \int_{0}^{1} d y \int_{0}^{y} d z[x(1-x)]^{-\epsilon / 2}(1-y)^{\epsilon / 2-1}(y-z) U_{131}^{-1-\epsilon}(x, y, z)
$$

take the derivative and put $p^{2}=-M^{2}$ obtaining

$S_{p b}^{131}=\left(\frac{\mu^{2}}{\pi}\right)^{\epsilon} \Gamma(2+\epsilon) \int_{0}^{1} d x \int_{0}^{1} d y \int_{0}^{y} d z[x(1-x)]^{-\epsilon / 2}(1-y)^{\epsilon / 2-1}(y-z) z(1-z) U_{\text {os; } 131}^{-2-\epsilon}(x, y, z)$,

where the quadratic form $U$ is

$$
U_{\mathrm{os} ; 131}(x, y, z)=M^{2} z^{2}+m_{x}^{2}(1-y), \quad m_{x}^{2}=\frac{m^{2}}{x(1-x)} .
$$

Now we make use of the identity

$$
z(1-z) U_{\mathrm{os} ; 131}^{-2-\epsilon}(x, y, z)=-\frac{1}{2(1+\epsilon) M^{2}}(1-z) \partial_{z} U_{\mathrm{os} ; 131}^{-1-\epsilon}(x, y, z)
$$

and integrate by parts obtaining

$$
S_{p b}^{131}=\frac{1}{2 M^{2}}\left(\frac{\mu^{2}}{\pi}\right)^{\epsilon} \Gamma(1+\epsilon) \int_{0}^{1} d x[x(1-x)]^{-\epsilon / 2}\left(\mathcal{S}_{b}+\mathcal{S}_{c}\right)
$$

where we have introduced

$$
\begin{aligned}
& \mathcal{S}_{b}=\left(m_{x}^{2}\right)^{-1-\epsilon} \int_{0}^{1} d y y(1-y)^{-2-\epsilon / 2}=\frac{4}{\epsilon(2+\epsilon)}\left(m_{x}^{2}\right)^{-1-\epsilon} \\
& \mathcal{S}_{c}=\int_{0}^{1} d y \int_{0}^{y} d z(1-y)^{\epsilon / 2-1}(2 z-1-y) U_{\text {os } ; 131}^{-1-\epsilon}(x, y, z) .
\end{aligned}
$$

In order to compute $\mathcal{S}_{c}$, we make the transformation $y=1-y^{\prime}$ and introduce the function

$$
E(\mu, \nu, \alpha)=\int_{0}^{1} d y \int_{0}^{1-y} d z y^{\mu} z^{\nu} U_{\mathrm{os} ; 131}^{\alpha}(x, y, z)
$$


which can be computed explicitly with the help of the identity

$$
\left[1-\frac{1}{\alpha}\left(y \partial_{y}+\frac{z}{2} \partial_{z}\right)\right] U_{\mathrm{os} ; 131}^{\alpha}(x, y, z)=0
$$

giving the following result:

$$
E(\mu, \nu, \alpha)=\frac{1}{2 \alpha+2 \mu+\nu+3} \int_{0}^{1} d y y^{\mu}(1-y)^{\nu}(1+y) U_{\mathrm{os} ; 131}^{\alpha}(x, y, 1-y)
$$

Substituting this result into Eq.(257) and expanding around $\epsilon=0$ we obtain

$$
S_{p b}^{131}==\frac{1}{2 M^{4}}\left(\frac{\mu^{2}}{\pi M^{2}}\right)^{\epsilon} \Gamma(1+\epsilon) \int_{0}^{1} d x \int_{0}^{1} d y\left[\mathcal{S}_{-2} \epsilon^{-2}+\mathcal{S}_{-1} \epsilon^{-1}+\mathcal{S}_{0}\right]
$$

where the coefficients $\mathcal{S}$ are given by

$$
\begin{aligned}
\mathcal{S}_{-2} & =-4, \quad \mathcal{S}_{-1}=2 X\left[\frac{y}{\chi}-\left(\frac{1}{y \chi}\right)_{+}\right]+\frac{1}{3 \mu^{2}} \\
\mathcal{S}_{0} & =X\left[\frac{3+y}{\chi}-2 \frac{y}{\chi} L+2\left(\frac{1}{y \chi}\right)_{+}+2 L_{+}\right]-4+\zeta(2)-\frac{1}{3 \mu^{2}}\left(\frac{4}{3}-\ln \mu^{2}\right),
\end{aligned}
$$

where we have introduced $\chi_{\mathrm{os} ; 131}=X(1-y)^{2}+\mu^{2} y, \mu^{2}=m^{2} / M^{2}, X=x(1-x)$ and

$$
L=\ln \chi-\frac{1}{2} \ln X-\frac{1}{2} \ln y, \quad L_{+}=\left(\frac{\ln \chi}{y \chi}\right)_{+}-\frac{1}{2}\left(\frac{1}{y \chi}\right)_{+}(\ln X+\ln y) .
$$

Note that we can treat these coefficients numerically since $\chi$ is a semi-positive defined quadratic form with zeros at $y=1$ and $x=0$ or $x=1$ which are compensated by the numerators (note that we have changed again variable, from $y$ to $1-y)$. A convenient trick consists in extracting these factors in order to obtain a manifestly finite expression. Consider, for instance, the following integral,

$$
\mathcal{I}=\int_{0}^{1} d x \int_{0}^{1} d y \frac{x^{2}(1-x)^{2}}{\chi^{2}}
$$

after some straightforward manipulation we obtain

$$
\begin{aligned}
\mathcal{I} & =\int_{0}^{1} d x \int_{0}^{1} d y\left[\frac{x^{2} y(1-x y)^{2}}{\chi_{1}^{2}}+\frac{x(1-x)}{\chi_{2}^{2}}+\frac{x^{2} y(1-x y)}{\chi_{3}^{2}}\right], \\
\chi_{1} & =x(1-x y)(1-y)^{2}+\mu^{2} \\
\chi_{2} & =[1-x y(1-x)]^{2}+\mu^{2} y \\
\chi_{3} & =x[1-y(1-x y)]^{2}+\mu^{2}
\end{aligned}
$$

where it is immediately seen that the three quadratic forms are strictly positive over the $[0,1]^{2}$ region. 
By looking at Eq. (150) we see that the evaluation of $S_{p b}^{121}$ is very similar to the one we have just performed. Using the same technique we obtain

$$
\begin{aligned}
S^{121} & =-2\left(\frac{\mu^{2}}{\pi}\right)^{\epsilon} \Gamma(\epsilon) \int_{0}^{1} d x \int_{0}^{1} d y \int_{0}^{y} d z[x(1-x)]^{-\epsilon / 2}(1-y)^{\epsilon / 2} \\
& \times\left[\left(m_{x}^{2}+z^{2} p^{2}\right) U_{121}^{-1-\epsilon}+\frac{2-\epsilon / 2}{\epsilon} U_{121}^{-\epsilon}\right]
\end{aligned}
$$

where the quadratic form now is

$$
U_{121}=-p^{2} z^{2}+\left(p^{2}+m^{2}\right) z+m_{x}^{2}(1-y)
$$

First we take the derivative with respect to $p^{2}$ and next we impose the on-shell condition, $p^{2}=$ $-m^{2}$, obtaining

$$
\begin{aligned}
S_{p b}^{121} & =-\frac{2}{m^{2}}\left(\frac{\mu^{2}}{\pi m^{2}}\right)^{\epsilon} \Gamma(\epsilon) \int_{0}^{1} d x \int_{0}^{1} d y \int_{0}^{y} d z[x(1-x)]^{-\epsilon / 2}(1-y)^{\epsilon / 2} \\
& \times\left.\left\{z(1-z)\left[(1+\epsilon)\left(z^{2}-X\right) \chi_{121}^{-2-\epsilon}+\frac{\epsilon}{2} \chi_{121}^{-1-\epsilon}\right]+z(3 z-2) \chi_{121}^{-1-\epsilon}\right\}\right|_{\text {os }} .
\end{aligned}
$$

where the on-shell quadratic form is

$$
\chi_{\mathrm{os} ; 121}=z^{2}+X(1-y), \quad X=\frac{1}{x(1-x)} .
$$

To proceed, we can use the following relation:

$$
\left[1+\frac{1}{1+\epsilon}\left(y \partial_{y}+z \partial_{z}\right)\right] \chi_{\mathrm{os} ; 121}^{-1-\epsilon}=-\left(z^{2}-X\right) \chi_{\mathrm{os} ; 121}^{-2-\epsilon}
$$

In this way we derive

$$
S_{p b}^{121}=\frac{\Gamma(\epsilon+1)}{m^{2}}\left(\frac{\mu^{2}}{\pi m^{2}}\right)^{\epsilon} \int_{0}^{1} d x \int_{0}^{1} d y \int_{0}^{y} d z[x(1-x)]^{-\epsilon / 2}(1-y)^{\epsilon / 2-1} z(1-z) \chi_{\mathrm{os} ; 121}^{-1-\epsilon}
$$

Now we can use

$$
z(1-z)\left(\chi_{\mathrm{os} ; 121}\right)^{-1-\epsilon}=-\frac{1}{2 \epsilon}(1-z) \partial_{z} \chi_{\mathrm{os} ; 121}^{-\epsilon} .
$$

Once the integration by parts is performed we introduce $y^{\prime}=1-y$ and it follows that

$$
S_{p}^{121}=-\frac{\Gamma(\epsilon)}{2 m^{2}}\left(\frac{\mu^{2}}{\pi m^{2}}\right)^{\epsilon} \int_{0}^{1} d x \int_{0}^{1} d y[x(1-x)]^{-\epsilon / 2} y^{\epsilon / 2-1}\left(y \chi_{y}^{-\epsilon}-\chi_{0}^{-\epsilon}+\int_{0}^{1-y} d z \chi^{-\epsilon}\right)(27
$$

where the various $\chi$ 's are expressed as

$$
\chi=z^{2}+X y, \quad \chi_{y}=(1-y)^{2}+X y, \quad \chi_{0}=X y .
$$


In order to perform the $z$-integration we use the function $E(\mu, \nu, \alpha)$ introduced for $S_{p b}^{131}$ in Eq.(259) and derive:

$$
\int_{0}^{1} d y \int_{0}^{1-y} d z(1-y)^{\epsilon / 2-1} \chi^{-\epsilon}=\frac{1}{1-\epsilon} \int_{0}^{1} d y y^{\frac{\epsilon}{2}-1}(1+y) \chi_{y}^{-\epsilon}
$$

After the integration of the $\chi_{0}$ term, we introduce $U_{y}=x(1-x) \chi_{y}=x(1-x)(1-y)^{2}+y$ and perform the $\epsilon$-expansion, obtaining:

$$
S_{p b}^{121}=-\frac{1}{2 m^{2}}\left(\frac{\mu^{2}}{\pi m^{2}}\right)^{\epsilon} \Gamma(1+\epsilon)\left(\mathcal{S}_{1}+\mathcal{S}_{2}\right)
$$

where the coefficients are

$$
\mathcal{S}_{1}=\frac{2}{\epsilon^{2}} \frac{\Gamma^{2}(1+\epsilon / 2)}{\Gamma(2+\epsilon)}, \quad \mathcal{S}_{2}=\frac{2}{\epsilon^{2}}+\frac{6}{\epsilon}-\int_{0}^{1} d x \int_{0}^{1} d y\left[2 \ln U_{y}+\left(\frac{\ln U_{y}}{y}\right)_{+}\right]+4-\frac{1}{2} \zeta(2) .
$$

\section{$7.5 \quad S^{221}$ topology}

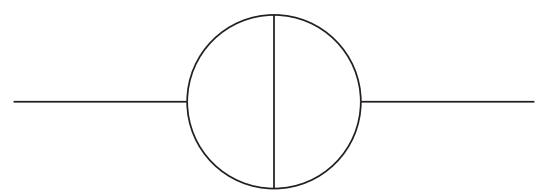

Figure 12: The two-loop diagram $S^{221}$ of Eq.(279).

The evaluation of $S^{221}$ is more involved than ones already presented for the other two-loop self-energies. We have

$$
\begin{aligned}
\pi^{4} S^{221} & =\mu^{2 \epsilon} \int d^{n} q_{1} d^{n} q_{2}\left(q_{1}^{2}+m_{1}^{2}\right)^{-1}\left(\left(q_{1}+p\right)^{2}+m_{2}^{2}\right)^{-1}\left(\left(q_{1}-q_{2}\right)^{2}+m_{3}^{2}\right)^{-1} \\
& \times\left(q_{2}^{2}+m_{4}^{2}\right)^{-1}\left(\left(q_{2}+p\right)^{2}+m_{5}^{2}\right)^{-1} .
\end{aligned}
$$

First of all the $\alpha-\gamma$ propagators are combined with parameters $x_{1}, x_{2}$. After performing the $q_{1}$-integration we introduce two additional parameters $y, z$, perform the $q_{2}$-integration and derive

$$
\begin{aligned}
S^{221} & =-\left(\frac{\mu^{2}}{\pi}\right)^{\epsilon} \Gamma(1+\epsilon) \int_{0}^{1} d x_{1} \int_{0}^{x_{1}} d x_{2}\left[x_{2}\left(1-x_{2}\right)\right]^{-1-\epsilon / 2} \\
& \times \int_{0}^{1} d y \int_{0}^{y} d z(1-y)^{\epsilon / 2} U_{221}^{-1-\epsilon} \\
U_{221} & =-\left[(1-y) p_{x}+z p\right]^{2}+z\left(p^{2}+m_{5}^{2}\right)+(y-z) m_{4}^{2}+(1-y) m_{x x}^{2}
\end{aligned}
$$


where $x_{1,2}$-dependent mass and momentum have been introduced:

$$
\begin{aligned}
p_{x} & =\frac{x_{1}-x_{2}}{1-x_{2}} p \\
m_{x x}^{2} & =\frac{1}{x_{2}\left(1-x_{2}\right)}\left[\left(x_{1}-x_{2}\right)\left(1-x_{1}+x_{2}\right) p^{2}+\left(1-x_{1}\right) m_{1}^{2}+\left(x_{1}-x_{2}\right) m_{2}^{2}+x_{2} m_{3}^{2}\right] .
\end{aligned}
$$

The quadratic form in $y, z$ has coefficients

$$
\begin{gathered}
H=\left(\begin{array}{cr}
-p_{x}^{2} & p \cdot p_{x} \\
p \cdot p_{x} & -p^{2}
\end{array}\right), \quad K^{t}=\frac{1}{2}\left(2 p_{x}^{2}+m_{4}^{2}-m_{x x}^{2}, p^{2}-2 p \cdot p_{x}+m_{5}^{2}-m_{4}^{2}\right) \\
L=m_{x x}^{2}-p_{x}^{2}-i \delta .
\end{gathered}
$$

Since $p_{x}$ and $p$ are simply related by

$$
p_{x}=X p, \quad X=\frac{x_{1}-x_{2}}{1-x_{2}}
$$

the matrix $\mathrm{H}$ is singular. To overcome this problem we introduce new variables $y=y^{\prime}$, and $z=z^{\prime}+X\left(y^{\prime}-1\right)$, giving for the quadratic form $U_{221}$ the simple expression $U_{221}=a z^{2}+b z+c y+d$ with coefficients

$$
\begin{array}{ll}
a=-p^{2} & b=p^{2}+m_{5}^{2}-m_{4}^{2}, \\
c=m_{4}^{2}-m_{x x}^{2}+X\left(p^{2}+m_{5}^{2}-m_{4}^{2}\right) & d=m_{x x}^{2}-X\left(p^{2}+m_{5}^{2}-m_{4}^{2}\right) .
\end{array}
$$

After the transformation we will use the identity

$$
\left[1+\frac{y}{\epsilon} \partial_{y}+\frac{1}{2 \epsilon}\left(z+\frac{b}{2 a}\right) \partial_{z}\right] U_{221}^{-\epsilon}=-\frac{B_{221}}{4 a} U_{221}^{-1-\epsilon} .
$$

with $B_{221}=b^{2}-4 a d$. This factor reads as follows:

$$
B_{221}=\lambda\left(-p^{2}, m_{4}^{2}, m_{5}^{2}\right)+4 p^{2}\left(m_{x x}^{2}-m_{4}^{2}\right)-4 X p^{2}\left(p^{2}+m_{5}^{2}-m_{4}^{2}\right)=s b_{221} .
$$

Before continuing in the evaluation of the diagram we study its singularities.

\subsection{Landau equations for $S^{221}$}

The Landau equations corresponding to $S^{221}$ are

$$
\begin{array}{ll}
\alpha_{1}\left(q_{1}^{2}+m_{1}^{2}\right)=0 & \alpha_{2}\left(\left(q_{1}+p\right)^{2}+m_{2}^{2}\right)=0 \\
\alpha_{3}\left(\left(q_{1}-q_{2}\right)^{2}+m_{3}^{2}\right)=0 & \alpha_{4}\left(q_{2}^{2}+m_{4}^{2}\right)=0 \\
\alpha_{5}\left(\left(q_{2}+p\right)^{2}+m_{5}^{2}\right)=0 &
\end{array}
$$


and also

$$
\begin{aligned}
& \alpha_{1} q_{1 \mu}+\alpha_{2}\left(q_{1}+p\right)_{\mu}+\alpha_{3}\left(q_{1}-q_{2}\right)_{\mu}=0, \\
& -\alpha_{3}\left(q_{1}-q_{2}\right)_{\mu}+\alpha_{4} q_{2 \mu}+\alpha_{5}\left(q_{2}+p\right)_{\mu}=0 .
\end{aligned}
$$

The leading Landau singularity occurs for $\alpha_{i} \neq 0, \forall i$. We multiply the two equations Eq.(287) by $q_{1 \mu}, q_{2 \mu}$ and $p_{\mu}$ respectively. This gives an homogeneous system of six equations. If all $\alpha_{i}$ are different from zero, the singularity will occur for

$$
\begin{array}{ll}
q_{1}^{2}=-m_{1}^{2} & q_{2}^{2}=-m_{4}^{2}, \\
q_{1} \cdot q_{2}=\frac{1}{2}\left(m_{3}^{2}-m_{1}^{2}-m_{4}^{2}\right) & p \cdot q_{2}=\frac{1}{2}\left(s+m_{4}^{2}-m_{5}^{2}\right), \\
p \cdot q_{1}=\frac{1}{2}\left(s+m_{1}^{2}-m_{2}^{2}\right) &
\end{array}
$$

The equations become as follows:

$$
\begin{aligned}
& -2 m_{1}^{2} \alpha_{1}+\left(s-m_{1}^{2}-m_{2}^{2}\right) \alpha_{2}-\left(m_{1}^{2}+m_{3}^{2}-m_{4}^{2}\right) \alpha_{3}=0 \\
& -\left(m_{1}^{2}-m_{3}^{2}+m_{4}^{2}\right) \alpha_{1}+\left(s-m_{1}^{2}+m_{3}^{2}-m_{5}^{2}\right) \alpha_{2}-\left(m_{1}^{2}-m_{3}^{2}-m_{4}^{2}\right) \alpha_{3}=0 \\
& \left(s+m_{1}^{2}-m_{2}^{2}\right) \alpha_{1}-\left(s-m_{1}^{2}+m_{2}^{2}\right) \alpha_{2}+\left(m_{1}^{2}-m_{2}^{2}-m_{4}^{2}+m_{5}^{2}\right) \alpha_{3}=0 \\
& \left(m_{1}^{2}+m_{3}^{2}-m_{4}^{2}\right) \alpha_{3}-\left(m_{1}^{2}-m_{3}^{2}+m_{4}^{2}\right) \alpha_{4}+\left(s-m_{2}^{2}+m_{3}^{2}-m_{4}^{2}\right) \alpha_{5}=0 \\
& \left(m_{1}^{2}-m_{3}^{2}-m_{4}^{2}\right) \alpha_{3}-2 m_{4}^{2} \alpha_{4}+\left(s-m_{4}^{2}-m_{5}^{2}\right) \alpha_{5}=0 \\
& -\left(m_{1}^{2}-m_{2}^{2}-m_{4}^{2}+m_{5}^{2}\right) \alpha_{3}+\left(s+m_{4}^{2}-m_{5}^{2}\right) \alpha_{4}-\left(s-m_{4}^{2}+m_{5}^{2}\right) \alpha_{5}=0 .
\end{aligned}
$$

In order to have non trivial solutions the determinants of the coefficients must be equal to zero. In both cases the solution is given by

$$
\begin{aligned}
s & =\frac{1}{2 m_{3}^{2}}\left\{m_{3}^{2}\left(m_{3}^{2}-m_{1}^{2}-m_{2}^{2}-m_{4}^{2}-m_{5}^{2}\right)-\left(m_{1}^{2}-m_{4}^{2}\right)\left(m_{5}^{2}-m_{2}^{2}\right)\right. \\
& \left. \pm\left[\lambda\left(m_{3}^{2}, m_{1}^{2}, m_{4}^{2}\right) \lambda\left(m_{3}^{2}, m_{2}^{2}, m_{5}^{2}\right)\right]^{1 / 2}\right\}
\end{aligned}
$$

which represents the so-called anomalous threshold for this topology, the leading Landau singularity. Actually the singularity occurs when the product of the two lambda-functions is positive, i.e for

$$
m_{3}^{2} \leq m_{01}^{2} \quad \text { or } \quad m_{02}^{2} \leq m_{3}^{2} \leq m_{03}^{2} \quad \text { or } \quad m_{3}^{2} \geq m_{04}^{2}
$$

where auxiliary masses are defines as

$$
\begin{aligned}
& m_{01}^{2}=\min \left\{\left(m_{1}-m_{4}\right)^{2},\left(m_{2}-m_{5}\right)^{2}\right\}, \\
& m_{02}^{2}=\min \left\{\max \left\{\left(m_{1}-m_{4}\right)^{2},\left(m_{2}-m_{5}\right)^{2}\right\}, \min \left\{\left(m_{1}+m_{4}\right)^{2},\left(m_{2}+m_{5}\right)^{2}\right\}\right\}, \\
& m_{03}^{2}=\max \left\{\max \left\{\left(m_{1}-m_{4}\right)^{2},\left(m_{2}-m_{5}\right)^{2}\right\}, \min \left\{\left(m_{1}+m_{4}\right)^{2},\left(m_{2}+m_{5}\right)^{2}\right\}\right\}, \\
& m_{04}^{2}=\max \left\{\left(m_{1}+m_{4}\right)^{2},\left(m_{2}+m_{5}\right)^{2}\right\},
\end{aligned}
$$

The sub-leading Landau singularities follow from a study of the reduced diagrams of Fig. 13. 

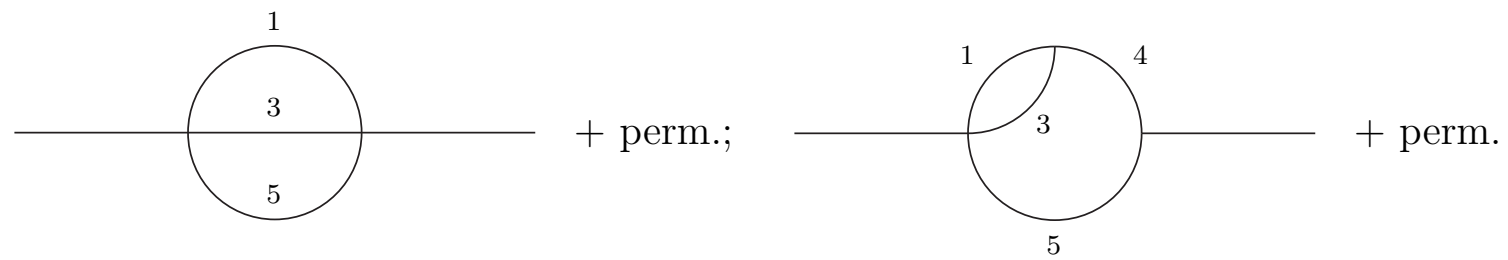

Figure 13: The reduced diagrams corresponding to the $S^{221}$ topology of Fig. 12. Their leading Landau singularities are the sub-leading ones for $S^{221}$.

\subsection{Evaluation of $S^{221}$ : method I}

To compute $S^{221}$ we must, first of all, considers the roots of $b_{221}$ defined in Eq.(286). It follows that $b_{221}=0$ for

$$
x_{1}^{2}+\left(\mu_{5}^{2}+\frac{1}{4} \lambda\right) x_{2}^{2}-\left(1+\mu_{5}^{2}-\mu_{4}^{2}\right) x_{1} x_{2}-\left(1+\mu_{1}^{2}-\mu_{2}^{2}\right) x_{1}+\left(1-\mu_{2}^{2}+\mu_{3}^{2}-\mu_{4}^{2}-\frac{1}{4} \lambda\right) x_{2}+\mu_{1}^{2}=0,
$$

where $\lambda=\lambda\left(1, \mu_{4}^{2}, \mu_{5}^{2}\right)$. Before discussing the general case let us consider some simple example, e.g. $m_{i}=m, \forall i \neq 3$ and three cases: $m_{3}=0, m_{3}=m$ and also $m_{3}=M$, where $M$ is some large mass, $M>m$. The curves corresponding to $B=0$ are given in Fig. 14 where the integration domain, $0 \leq x_{1} \leq 1$ and $0 \leq x_{2} \leq x_{1}$ is also shown. Before raising powers we introduce $U_{221}=s \chi_{221}$, with

$$
\chi_{221}=z^{2}-\mu_{45}^{2} z+\left(\mu_{4}^{2}-\mu_{x x}^{2}-X \mu_{45}^{2}\right) y+\mu_{x x}^{2}+X \mu_{45}^{2},
$$

where, as usual, $\mu_{i j}^{2}=1+\mu_{i}^{2}-\mu_{j}^{2}$. The raising procedure requires

$$
\chi_{221}^{-1-\epsilon}=-\frac{4}{b_{221}}\left[1+\frac{y}{\epsilon} \partial_{y}+\frac{1}{2 \epsilon}(z+Z) \partial_{z}\right] \chi_{221}^{-\epsilon},
$$

where the object of interest is

$$
b_{221}=\lambda\left(1, \mu_{4}^{2}, \mu_{5}^{2}\right)-4\left(\mu_{x x}^{2}-\mu_{4}^{2}\right)-4 X \mu_{45}^{2}, \quad Z=-\frac{1}{2} \mu_{45}^{2}, \quad X=\frac{x_{1}-x_{2}}{1-x_{2}},
$$

and $0 \leq y \leq 1$, with $X(1-y) \leq z \leq y+X(1-y)$. After a Laurent expansion in $\epsilon$ we will have terms proportional to powers of $\ln \chi_{221}$; following these operation we substitute back $z=z^{\prime}-X(y-1)$, so that the order of integration will be specified as

$$
\int_{0}^{1} d y \int_{0}^{y} d z \int_{0}^{1} d x_{2} \int_{x_{2}}^{1} d x_{1}
$$






Figure 14: The $x_{1}-x_{2}$ plane with the curves $b_{221}=0$ where $b_{221}$ is defined in Eq.(286). Here $m_{i}=$ $m, \forall i \neq 3$ and $m_{3}=0, m, M$ with $M>m$. 
The argument of the logarithm is

$$
\chi_{221}=\frac{X_{221}}{x_{2}\left(1-x_{2}\right)^{2}}, \quad X_{221}=A_{\xi} x_{1}^{2}+B_{\xi} x_{1}+C_{\xi},
$$

where the coefficients $A_{\xi}-C_{\xi}$ are given by

$$
\begin{aligned}
& A_{\xi}^{0}=(1-y)\left(1-x_{2}\right), \quad B_{\xi}^{0}=-(1-y)\left(1-x_{2}\right)\left(\mu_{54}^{2} x_{2}+\mu_{12}^{2}\right), \\
& C_{\xi}^{0}=x_{2}\left(1-x_{2}\right)^{2}\left(z^{2}-\mu_{45}^{2} z+\mu_{4}^{2} y\right)-(1-y)\left(1-x_{2}\right)\left[\left(\mu_{4}^{2}-\mu_{5}^{2}\right) x_{2}^{2}-\mu_{32}^{2} x_{2}-\mu_{1}^{2}\right],
\end{aligned}
$$

and $\mu_{i j}^{2}=1+\mu_{i}^{2}-\mu_{j}^{2}$. Zeros of $b_{221}$ are avoided by distorting the $x_{1}$ integration contour in a way that preserves the correct imaginary part of $\ln X_{221}$. As done for the sunset diagram we consider the two roots of the equation $X_{221}=0$, say $x_{1\{L, R\}}$. If the roots are complex conjugated we select a contour which starts at $x_{1}=x_{2}$ and bypasses the zeros of $b_{221}$ in the upper (or lower) half-plane to return to $x_{1}=1$.

If the roots are real we must distinguish between two cases: when $A_{\xi}$ is positive then the cut is between $x_{1 L}$ and $x_{1 R}$, while for negative $A_{\xi}$, the cut is $\left[-\infty, x_{1 L}\right] \cup\left[x_{1 R},+\infty\right]$. Furthermore, with $x_{1}=\alpha+i \beta$, the imaginary part of the logarithm is $\beta\left(2 \alpha A_{\xi}+B_{\xi}\right)$. This consideration immediately tells us the sign of the imaginary part when $x_{1}$ approaches the real axis on the cut. This sign is crucial when we distort the contour in the complex plane since, for $x_{1}$ real and on the cut, the $i \delta$ prescription, gives $-i \pi$ for the imaginary part.

Let us consider one of the normal thresholds, $s=\left(m_{1}+m_{3}+m_{5}\right)^{2}$, as we have done for other diagrams. This corresponds to the three-particle cuts of Fig. 15. Let $x_{1\{L, R\}}$ be the two points where $X_{221}=0$ and $x_{1 \pm}$ the two roots of $b_{221}=0$. Whenever $x_{1 \pm}$ are on the real axis we want to distort the integration contour.

In Fig. 16 we give the real and imaginary parts of these four points as a function of $x_{2}$ for fixed $y$ and different values of $z$. For decreasing $x_{2}$ the branch points and $x_{1 \pm}$ are complex conjugated and no distortion is needed. For values of $z$ near to $y$ the branch points become real when $x_{1 \pm}$ are still complex and, again, distortion is not needed till the value of $x_{2}$ where $x_{1 \pm}$ are real. However, for some value of $z$, that we call $z_{\text {th }}(y)$ the following happens: the two branch points pinch the real $x_{1}$-axis exactly at $x_{1+} \equiv x_{1-}$ and distortion is not allowed anymore. A part for having a larger number of Feynman parameters, the situation is completely similar to the one described for the sunset diagram [7]. Since there is no ultraviolet divergency we may set $\epsilon=0$, obtaining

$$
S^{221}=-\frac{1}{s} \int_{0}^{1} d x_{1} \int_{0}^{x_{1}} d x_{2}\left(1-x_{2}\right) \int_{0}^{1} d y \int_{X(1-y)}^{y+X(1-y)} d z X_{221}^{-1} .
$$

Our first step will be to use the following relation:

$$
\chi_{221}^{-1}=-\frac{4}{b_{221}}\left\{1-\left[y \partial_{y}+\frac{1}{2}(z+Z) \partial_{z}\right] \ln \chi_{221}\right\} .
$$

We obtain

$$
S^{221}=\frac{4}{b_{221}^{R}} \int_{0}^{1} d x_{1} \int_{0}^{x_{1}} d x_{2}\left(1-x_{2}\right) \int_{0}^{1} d y \int_{X(1-y)}^{y+X(1-y)} d z\left\{1-\left[y \partial_{y}+\frac{1}{2}(z+Z) \partial_{z}\right] \ln \chi_{221}\right\} .
$$






Figure 15: The three-particle cut of $S^{221}$.

where $b_{221}^{R}=x_{2}\left(1-x_{2}\right) \Lambda$. Three new quantities are needed:

$$
\begin{aligned}
& \chi_{221}^{1}=\chi_{221}(z=y+X(1-y))=(1-X)^{2} y^{2}-\left[1-\mu_{5}^{2}+\mu_{x x}^{2}-2 X(1-X)\right] y+\mu_{x x}^{2}+X^{2}, \\
& \chi_{221}^{2}=\chi_{221}(z=X(1-y))=X^{2} y^{2}+\left[\mu_{4}^{2}-\mu_{x x}^{2}-2 X^{2}\right] y+\mu_{x x}^{2}+X^{2}, \\
& \chi_{221}^{3}=\chi_{221}(y=1, z=y)=y^{2}-\mu_{45}^{2} y+\mu_{4}^{2} .
\end{aligned}
$$

Therefore, using

$$
\int_{0}^{1} d y \int_{X(1-y)}^{y+X(1-y)} d z=\int_{0}^{X} d z \int_{1-\frac{z}{X}}^{1} d y+\int_{X}^{1} d z \int_{\frac{z-X}{1-X}}^{1} d y
$$

we obtain

$$
\begin{aligned}
S^{221} & =\frac{4}{s b_{221}^{R}} \int_{0}^{1} d x_{1} \int_{0}^{x_{1}} d x_{2}\left\{\frac{3}{2} \int_{0}^{1} d y \int_{X(1-y)}^{y+X(1-y)} d z \ln \chi_{221}-\int_{0}^{1} d y \ln \chi_{221}^{3}\right. \\
& \left.-\frac{1}{2} \int_{0}^{1} d y\left[(X-y+X y+Z) \ln \chi_{221}^{1}-(X+X y+Z) \ln \chi_{221}^{2}\right]+\frac{1}{2}\right\}
\end{aligned}
$$

Next we change variable, $z \rightarrow z^{\prime}$, so that $0 \leq z^{\prime} \leq 1$ and write all $\chi^{\prime}$ s as polynomials in $x_{1}$ with coefficients that are functions of $x_{2}, y$ and $z$. In particular we introduce

$$
\chi_{221}^{1}=\frac{X_{221}^{1}}{x_{2}\left(1-x_{2}\right)^{2}}, \quad X_{221}^{1}=A_{\xi}^{1} x_{1}^{2}+B_{\xi}^{1} x_{1}+C_{\xi}^{1} .
$$

we find

$$
\begin{gathered}
A_{\xi}^{1}=Y\left[1-x_{2}+Y x_{2}\right], \quad B_{\xi}^{1}=-Y\left[\left(1-x_{2}\right) \mu_{12}^{2}+2 Y x_{2}\right], \\
C_{\xi}^{1}=Y\left(1-x_{2}\right)\left(-2 x_{2}^{2} y+x_{2} \mu_{32}^{2}+x_{2}^{2}+\mu_{1}^{2}\right)+Y^{2} x_{2}^{3}+x_{2}\left(1-x_{2}\right)^{2}\left(\mu_{5}^{2}-Y\right) y \\
\chi_{221}^{2}=\frac{X_{221}^{2}}{x_{2}\left(1-x_{2}\right)^{2}}, \quad X_{221}^{2}=A_{\xi}^{2} x_{1}^{2}+B_{\xi}^{2} x_{1}+C_{\xi}^{2} . \\
A_{\xi}^{2}=Y\left(1-x_{2}+Y x_{2}\right), \quad B_{\xi}^{2}=-Y\left[\left(1-x_{2}\right)\left(\mu_{12}^{2}+2 x_{2}\right)+2 Y x_{2}^{2}\right] \\
C_{\xi}^{2}=Y\left(1-x_{2}\right)\left(\mu_{32}^{2} x_{2}+x_{2}^{2}+\mu_{1}^{2}\right)+Y^{2} x_{2}^{3}+x_{2}\left(1-x_{2}\right)^{2} \mu_{4}^{2} y .
\end{gathered}
$$



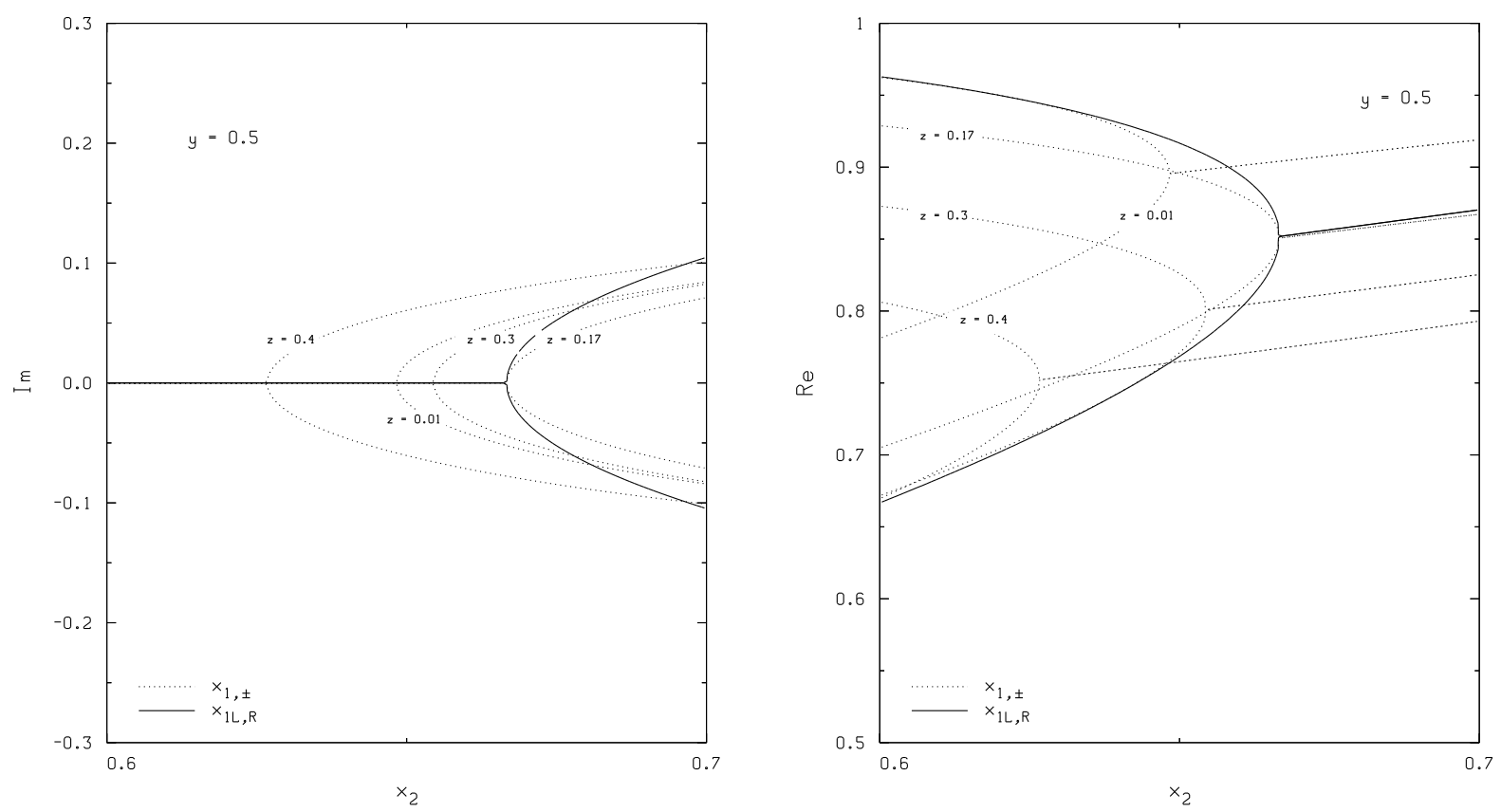

Figure 16: Behavior, at the normal threshold $s=\left(m_{1}+m_{3}+m_{5}\right)^{2}$ of branch points and apparent singularities for $S^{221}$ as a function of $x_{2}$. 
Finally we introduce

$$
\chi_{221}^{0}=\frac{X_{221}^{0}}{x_{2}\left(1-x_{2}\right)^{2}}, \quad X_{221}^{0}=A_{\xi}^{0} x_{1}^{2}+B_{\xi}^{0} x_{1}+C_{\xi}^{0},
$$

where

$$
\begin{aligned}
& A_{\xi}^{0}=(1-y)\left(1-x_{2}\right), \quad B_{\xi}^{0}=-(1-y)\left(1-x_{2}\right)\left(\mu_{54}^{2} x_{2}+\mu_{12}^{2}\right), \\
& C_{\xi}^{0}=x_{2}\left(1-x_{2}\right)^{2}\left(z^{2}-\mu_{45}^{2} z+\mu_{4}^{2} y\right)-(1-y)\left(1-x_{2}\right)\left[\left(\mu_{4}^{2}-\mu_{5}^{2}\right) x_{2}^{2}-\mu_{32}^{2} x_{2}-\mu_{1}^{2}\right] .
\end{aligned}
$$

Here we used

$$
Y=1-y \text {. }
$$

At this point we must realize that the situation for $S^{221}$ is different from all previous cases. Indeed $S^{221}$ and all its sub-diagrams are convergent and, for this reason, only logarithms appear after the first iteration and not logarithms squared. Therefore, the strategy for distorting the $x_{1}$ integration contour will be the following. Let us introduce variables $\Delta_{i}=x_{1}-x_{1 i}$, where $x_{1 i}$ is any root of the argument of a logarithm, and split the logarithms according to

$$
\ln \left(A_{\xi}^{n} x_{1}^{2}+B_{\xi}^{n} x_{1}+C_{\xi}^{n}-i \delta\right)=\ln A_{\xi}^{n}+\eta\left(A_{\xi}^{n}, \Delta_{L} \Delta_{R}\right)+\eta\left(\Delta_{L}, \Delta_{R}\right)+\sum_{i=L, R} \ln \left(x_{1}-x_{1 i}\right),
$$

where $\eta$ is the usual Veltman's function, $\eta(a, b)=\ln (a b)-\ln a-\ln b$, and logarithms are defined with a cut along the negative real axis. The final expression for the diagram will be the sum of terms proportional to $\eta$-functions and terms of the form $\ln \left(x_{1}-x_{1 i}\right) / \Lambda_{R}$. The former do not create a problem in distorting, when needed, the integration contour. For the latter, however, if one of the roots of $\lambda_{R}$, or both, is real and internal to the interval $[0,1]$ then the distortion for the corresponding term must be examined with care. If the imaginary part of $x_{1 i}$ is positive(negative) then we move the contour into the negative(positive) imaginary half-plane, so that the cut of the logarithm will never be crossed. If $x_{1 i}$ is real and negative no problem will arise and we can distort in any of the two ways. If its is real and positive then the distortion is fixed according to the $i \delta$ prescription: for instance the roots of $a x_{1}^{2}+b x_{1}+c-i \delta$ corresponding to $b^{2}-4 a c>0$ are such that

$$
\operatorname{Im} x_{1,\{L, R\}}=\mp \operatorname{sign}(a) \delta .
$$

The reason why we cannot apply this argument to the other cases discussed before is linked to the presence of $l^{2}$-terms. After the splitting of the logarithm we will encounter terms proportional to $\ln \left(x_{1}-x_{1 L}\right) \ln \left(x_{1}-x_{1 R}\right)$. When the roots are complex conjugated we will have cuts both in the positive and in the negative imaginary half-planes and the possibility of distorting the contour ceases when they approach the real axis. 


\subsection{Evaluation of $S^{221}$ : method II}

An alternative evaluation of $S^{221}$ follows when we change variable in Eq.(280), $y=1-y^{\prime}$, so that

$$
\begin{aligned}
& \int_{0}^{1} d y \int_{X(1-y)}^{y+X(1-y)} d z(1-y)^{\epsilon / 2}\left(a z^{2}+b z+c y+d\right)^{-1-\epsilon}= \\
& \int_{0}^{1} d y \int_{X y}^{1-(1-X) y} d z y^{\epsilon / 2}\left(a z^{2}+b z-c y+d^{\prime}\right)^{-1-\epsilon} .
\end{aligned}
$$

This method has the advantage that $d^{\prime}=c+d=m_{4}^{2}$, with a corresponding change of $b_{221} \rightarrow b_{221}^{\prime}$, where $b_{221}^{\prime}=\mu_{45}^{4}-4 \mu_{4}^{2}$, is $x_{1,2}$-independent. In this way the $x_{1}-x_{2}$ integration is free of numerical instabilities. The only point in the external parameter space where a failure will occur is for $s=\left(m_{4} \pm m_{5}\right)^{2}$, representing normal and pseudo thresholds associated to the two-particle cut of Fig. 17. They are non-leading Landau singularities. The fact that $b_{221}^{\prime}$ is $x$ independent means that, with this method, we can perform the $x$-integration without having to distort the integration contour. As observed the method fails at the non-leading Landau singularities corresponding to the one-loop complementary sub-diagram of $S^{221}$ (in this case there are two sub-diagrams with the same number of lines). When we are in this kinematical configuration and $s \neq\left(m_{1} \pm m_{2}\right)^{2}$ the strategy will be to interchange the order of the operations, $q_{1} \leftrightarrow q_{2}$. When $\left(m_{1} \pm m_{2}\right)^{2}=\left(m_{4} \pm m_{5}\right)^{2}$ the method fails. Using this approach, we set $\epsilon=0$ and get

$$
\begin{gathered}
S^{221}=-\frac{1}{s} \int_{0}^{1} d x_{1} \int_{0}^{x_{1}} d x_{2} \int_{0}^{1} d y \int_{X y}^{1+(X-1) y} d z \\
\times\left[x_{2}\left(1-x_{2}\right)\right]^{-1} \chi_{221}^{-1}\left(x_{1}, x_{2}, 1-y, z-X y\right), \\
\chi_{221}\left(x_{1}, x_{2}, 1-y, z-X y\right)=z^{2}-\mu_{45}^{2} z+\left(\mu_{x x}^{2}-\mu_{4}^{2}+X \mu_{45}^{2}\right) y+\mu_{4}^{2} .
\end{gathered}
$$

Successively we use the identity

$$
\chi_{221}^{-1-\epsilon}=-\frac{4}{\lambda\left(1, \mu_{4}^{2}, \mu_{5}^{2}\right)}\left[1+\frac{y}{\epsilon} \partial_{y}+\frac{z-\mu_{45}^{2} / 2}{2 \epsilon} \partial_{z}\right] \chi_{221}^{-\epsilon} .
$$

The derivation continues, as usual, with an integration by parts, where we use

$$
\int_{0}^{1} d y \int_{X y}^{1+(X-1) y} d z=\int_{0}^{X} d z \int_{0}^{z / X} d y+\int_{X}^{1} d z \int_{0}^{(z-1) /(X-1)} d y
$$

Next we define

$$
\mu_{x x}^{2}=\frac{\nu_{x x}^{2}}{x_{2}\left(1-x_{2}\right)}
$$


and introduce four quadratic forms:

$$
\begin{aligned}
\chi_{221}^{1} & =x_{2}\left(x_{1}-x_{2}\right)\left(1-x_{2}\right) y \mu_{45}^{2}+x_{2}\left(1-x_{2}\right)\left(1-x_{1}\right) y\left(\mu_{45}^{2}-2\right) \\
& +x_{2}\left(1-x_{2}\right)^{2}\left(1-y \mu_{4}^{2}+\mu_{4}^{2}-\mu_{45}^{2}\right)+x_{2}\left(1-x_{1}\right)^{2} y^{2}+\left(1-x_{2}\right) y \nu_{x x}^{2}, \\
\chi_{221}^{2} & =x_{2}\left(x_{1}-x_{2}\right)^{2} y^{2}+x_{2}\left(1-x_{2}\right)^{2} \mu_{4}^{2}(1-y)+\left(1-x_{2}\right) y \nu_{x x}^{2}, \\
\chi_{221}^{3} & =x_{2}\left(x_{1}-x_{2}\right)\left(1-x_{2}\right) y \mu_{45}^{2}+x_{2}\left(1-x_{2}\right)\left(1-x_{1}\right) z\left(\mu_{45}^{2}-2\right) \\
& +x_{2}\left(1-x_{2}\right)^{2}\left(1-y \mu_{4}^{2}+\mu_{4}^{2}-\mu_{45}^{2}\right)+x_{2}\left(1-x_{1}\right)^{2} z^{2}+\left(1-x_{2}\right) y \nu_{x x}^{2}, \\
\chi_{221}^{4} & =x_{2}\left(x_{1}-x_{2}\right)\left(1-x_{2}\right) \mu_{45}^{2}(y-z)+x_{2}\left(x_{1}-x_{2}\right)^{2} z^{2} \\
& +x_{2}\left(1-x_{2}\right)^{2} \mu_{4}^{2}(1-y)+\left(1-x_{2}\right) y \nu_{x x}^{2} .
\end{aligned}
$$

Note that, in the limit $x_{2} \rightarrow 1$, we obtain

$$
\chi_{221}^{1}=\chi_{221}^{2}=\left(1-x_{1}\right)^{2} y^{2}, \quad \chi_{221}^{3}=\chi_{221}^{4}=\left(1-x_{1}\right)^{2} z^{2} .
$$

Similarly, in the limit $x_{2} \rightarrow 0$, we obtain $\chi_{221}^{i}=\nu_{x x}^{2} y$. The result for $S^{221}$ is as follows:

$$
S^{221}=-\frac{4}{s \lambda\left(1, \mu_{4}^{2}, \mu_{5}^{2}\right)} \int_{0}^{1} d x_{1} \int_{0}^{x_{1}} d x_{2} \frac{1}{x_{2}} J_{221}\left(x_{1}, x_{2}, y, z\right),
$$

where the function $J_{221}$ is

$$
J_{221}=\int_{0}^{1} d y \int_{y}^{1} d z J_{221}^{2}+\int_{0}^{1} d y J_{221}^{1}
$$

and

$$
\begin{aligned}
J_{221}^{1} & =\frac{1}{2} \frac{y\left(1-x_{1}\right)}{\left(1-x_{2}\right)^{2}}\left[\ln \chi_{221}^{1}-\ln \chi_{221}^{2}\right] \\
& +\frac{1}{1-x_{2}}\left[\frac{1}{2}\left(1-\frac{1}{2} \mu_{45}^{2}\right) \ln \chi_{221}^{1}+\frac{1}{2}\left(y+\frac{1}{2} \mu_{45}^{2}\right) \ln \chi_{221}^{2}\right], \\
J_{221}^{2} & =\frac{3}{2} \frac{1-x_{1}}{\left(1-x_{2}\right)^{2}}\left[\ln \chi_{221}^{4}-\ln \chi_{221}^{3}\right]-\frac{1}{1-x_{2}}\left[\frac{3}{2} \ln \chi_{221}^{4}+1\right] .
\end{aligned}
$$

It is straightforward to show that in the limit $x_{2}=0$

$$
J_{221}\left(x_{1}, 0, y, z\right)=0
$$

so that we obtain

$$
S^{221}=-\frac{4}{s \lambda\left(1, \mu_{4}^{2}, \mu_{5}^{2}\right)} \int_{0}^{1} d x_{1} \int_{0}^{x_{1}} d x_{2}\left(\frac{I^{221}\left(x_{1}, x_{2}\right)}{x_{2}}\right)_{+} .
$$


The integrand $I_{+}^{221}$ is defined as follows:

$$
I_{+}^{221}=\int_{0}^{1} d y \int_{y}^{1} d z I_{221}^{2}+\int_{0}^{1} d y I_{221}^{1}
$$

with auxiliary functions $I_{221}^{(1,2)}$ given by

$$
\begin{aligned}
I_{221}^{2} & =-\frac{x_{2}}{1-x_{2}}-\frac{3}{2}\left(1-x_{1}\right)\left[\frac{1}{\left(1-x_{2}\right)^{2}} \ln \chi_{221}^{3}\left(x_{1}, x_{2}, y, z\right)-\ln \chi_{221}^{3}\left(x_{1}, 0, y, z\right)\right] \\
& +\frac{3}{2}\left(1-x_{1}\right)\left[\frac{1}{\left(1-x_{2}\right)^{2}} \ln \chi_{221}^{4}\left(x_{1}, x_{2}, y, z\right)-\ln \chi_{221}^{4}\left(x_{1}, 0, y, z\right)\right] \\
& -\frac{3}{2}\left[\frac{1}{1-x_{2}} \ln \chi_{221}^{4}\left(x_{1}, x_{2}, y, z\right)-\ln \chi_{221}^{4}\left(x_{1}, 0, y, z\right)\right], \\
I_{221}^{1}= & \frac{1}{2} y\left(1-x_{1}\right)\left[\frac{1}{\left(1-x_{2}\right)^{2}} \ln \chi_{221}^{1}\left(x_{1}, x_{2}, y\right)-\ln \chi_{221}^{1}\left(x_{1}, 0, y\right)\right] \\
& -\frac{1}{2} y\left(1-x_{1}\right)\left[\frac{1}{\left(1-x_{2}\right)^{2}} \ln \chi_{221}^{2}\left(x_{1}, x_{2}, y\right)-\ln \chi_{221}^{2}\left(x_{1}, 0, y\right)\right] \\
& +\frac{1}{2}\left(1-\frac{1}{2} \mu_{45}^{2}\right)\left[\frac{1}{1-x_{2}} \ln \chi_{221}^{1}\left(x_{1}, x_{2}, y\right)-\ln \chi_{221}^{1}\left(x_{1}, 0, y\right)\right], \\
& +\frac{1}{2}\left(y+\frac{1}{2} \mu_{45}^{2}\right)\left[\frac{1}{1-x_{2}} \ln \chi_{221}^{2}\left(x_{1}, x_{2}, y\right)-\ln \chi_{221}^{2}\left(x_{1}, 0, y\right)\right] .
\end{aligned}
$$

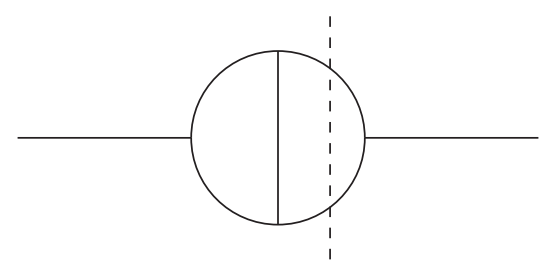

Figure 17: One of the two-particle cuts of $S^{221}$.

\subsubsection{The case $s=\left(m_{4} \pm m_{5}\right)^{2}$}

As already pointed out the derivation fails in this case because $\mu_{45}^{2}= \pm 2 \mu_{4}$. The alternative solution is quite similar to the one adopted for other topologies. If $\mu_{45}^{2}=-2 \mu_{4}$ we can change variable $z^{\prime}=z+\mu_{4}$ so that

$$
\chi_{221} \rightarrow z^{2}+\left(\mu_{x x}^{2}-\mu_{4}^{2}-2 X \mu_{4}\right) y,
$$

with $z_{-} \leq z \leq z_{+}$and

$$
z_{-}=X y+\mu_{4}, \quad z_{+}=1+(X-1) y+\mu_{4},
$$


where $z_{ \pm} \geq 0$. We change again variable $z^{2}=t$ and use

$$
(t+a y)^{-1}=\partial_{t} \ln (t+a y)
$$

and integrate by parts obtaining

$$
\begin{aligned}
\frac{1}{2} \int_{0}^{1} d y \int_{z_{-}^{2}}^{z_{+}^{2}} d t t^{-1 / 2} \partial_{t} \ln (t+a y) & =\frac{1}{2} \int_{0}^{1} d y\left[\frac{1}{z_{+}} \ln \left(z_{+}^{2}+a y\right)\right. \\
& \left.-\frac{1}{z_{-}} \ln \left(z_{-}^{2}+a y\right)+\frac{1}{2} \int_{z_{-}^{2}}^{z_{+}^{2}} d t t^{-3 / 2} \ln (t+a y)\right]
\end{aligned}
$$

If $\mu_{45}^{2}=2 \mu_{4}$ this derivation is of little use because the new limits of integration can be negative. However, in both cases we can write

$$
\mu_{4}^{2}=\frac{1}{4}\left[\mu_{45}^{4}-\lambda\left(1, \mu_{4}^{2}, \mu_{5}^{2}\right)\right]
$$

introduce $z_{m}=\mu_{45}^{2} / 2$ and transform $z^{\prime}=z-z_{m}$. With $z_{-}=X y-z_{m}$, and $z_{+}=1+(X-1) y-z_{m}$ we obtain

$$
\int_{0}^{1} d y \int_{z_{-}}^{z_{+}} d z\left(z^{2}+a y-\frac{1}{4} \lambda\right)^{-1}
$$

which gives

$$
\frac{1}{2} \int_{0}^{1} d y R^{-1}\left[\ln \frac{z_{+}-R}{z_{-}-R}-\ln \frac{z_{+}+R}{z_{-}+R}\right]
$$

where we have introduced

$$
R^{2}=\frac{1}{4} \lambda-a y, \quad a=\mu_{x x}^{2}-\mu_{4}^{2}-2 X \mu_{2}
$$

showing that no singularity will appear for $\lambda \equiv \lambda\left(1, \mu_{4}^{2}, \mu_{5}^{2}\right)=0$.

\subsection{Derivative of $S^{221}$ and infrared poles}

In this section we study another infrared divergent object, the on-shell derivative of the $S^{221}$ integral corresponding to the configuration a) where

$$
\text { a) } m_{2}=m_{4}=0, m_{1}=m_{3}=m_{5}=m \quad \text { and } \quad p^{2}=-m^{2} \text {. }
$$

It is important to recall that a necessary condition for the presence of infrared divergences is that the Landau equations are fulfilled. In the case of $S^{221}$ we see that the system of Landau equations, Eq.(288) is satisfied for this configuration of parameters if $\alpha_{3}=-\alpha_{1}$ and $\alpha_{5}=\alpha_{1}$. $S^{221}$ itself is not infrared divergent but the on-shell derivative is. Let us consider the diagram of Fig. 18 that

corresponds to configuration a). Solid lines correspond to the particle of mass $m$, while dashed 
lines give the massless particle. After taking the derivative with respect to $p^{2}$ we will have two contributions where the propagators $\left(q_{1}-p\right)^{2}+m^{2}$ and $\left(q_{2}+p\right)^{2}+m^{2}$ will appear with power -2 respectively. To study qualitatively the infrared behavior we neglect $q_{1}^{2}, q_{2}^{2}$ and $q_{1} \cdot q_{2}$ compared with $p \cdot q_{1}$ and $p \cdot q_{2}$ in propagator denominators, the so-called eikonal approximation. We further define a superficial degree of infrared divergence for an arbitrary scalar two-loop diagram

$$
\mu_{I R}(G)=2 n-1-2 n_{m}-n_{M},
$$

where $n_{m}$ is the number of massless lines and $n_{M}$ the number of massive lines. For the diagram of Fig. 18 (after $p^{2}$-differentiation and for on-shell $p$ ) we obtain $\mu_{I R}\left(S_{p}^{221}\right)=-1$. This shows the presence of an infrared pole singularity. We have

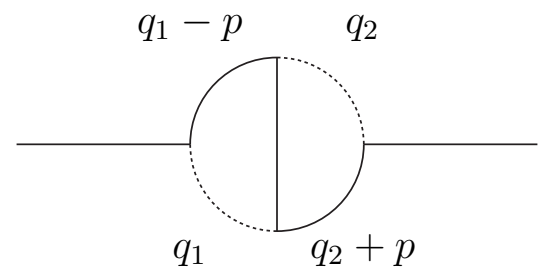

Figure 18: A special case of $S^{221}$ topology. Solid lines denote a massive particle, dashed lines a massless one.

$$
\begin{aligned}
S_{p}^{221} & =\frac{\partial}{\partial p^{2}} S^{221}=-\left(\frac{\mu^{2}}{\pi}\right)^{\epsilon} \Gamma(2+\epsilon) \int_{0}^{1} d x_{1} \int_{0}^{x_{1}} d x_{2}\left[x_{2}\left(1-x_{2}\right)\right]^{-1-\epsilon / 2} \\
& \times \int_{0}^{1} d y \int_{X y}^{1+(X-1) y} d z y^{\epsilon / 2}[z(z-1)-\rho y] U_{221}^{-2-\epsilon}
\end{aligned}
$$

where the quadratic form $U_{221}$ is defined by

$$
\begin{gathered}
U_{221}=-p^{2} z^{2}+\left(p^{2}+m^{2}\right)(z-X y)+m_{x x}^{2} y, \\
X=\frac{x_{1}-x_{2}}{1-x_{2}}, \quad \rho=\frac{1-x_{1}}{x_{2}} X,
\end{gathered}
$$

and $m_{x x}^{2}$ is given in Eq.(281). Next we define the on-shell limit of $U_{221}$,

$$
U_{221}\left(p^{2}=-m^{2}\right)=m^{2} \chi_{\mathrm{os} ; 221}, \quad \chi_{\mathrm{os} ; 221}=z^{2}+\eta y, \quad \eta=\frac{\left(1-x_{1}+x_{2}\right)^{2}}{x_{2}\left(1-x_{2}\right)} .
$$

Inserting this result into Eq. 342 we obtain the on-shell limit of the derivative, expressed as

$$
\begin{aligned}
S_{p}^{221 ; \mathrm{os}} & =-\left(\frac{\mu^{2}}{\pi m^{2}}\right)^{\epsilon} \Gamma(2+\epsilon) \frac{1}{m^{4}} \int_{0}^{1} d x_{1} \int_{0}^{x_{1}} d x_{2}\left[x_{2}\left(1-x_{2}\right)\right]^{-1-\epsilon / 2} \mathcal{S}_{221}, \\
\mathcal{S}_{221} & =\int_{0}^{1} d y \int_{X y}^{1+(X-1) y} d z y^{\epsilon / 2}[z(z-1)-\rho y]\left(z^{2}+\eta y\right)^{-2-\epsilon} .
\end{aligned}
$$


In the evaluation we must keep $\epsilon \neq 0$ and one cannot expand around $\epsilon=0$ after the $y-z$ integration because of the presence of overlapping infrared divergences; here we face a novel feature and the procedure is made even more complicated. Our solution is derived by adapting the general algorithm of [17]. Before explaining in detail how the residues of the infrared poles and the finite part can be cast into a form which allows for a reliable numerical integration we notice that the $z$-integration can be performed in Eq.(346) after using the identity:

$$
\left[1-\frac{1}{\mu}\left(y \partial_{y}+\frac{z}{2} \partial_{z}\right)\right]\left(z^{2}+\eta y\right)^{\mu}=0 .
$$

We define the following integrals:

$$
I_{i j}=\int_{0}^{1} d y \int_{X y}^{1+(X-1) y} d z y^{\epsilon / 2} z^{i} y^{j}\left(z^{2}+\eta y\right)^{-2-\epsilon}
$$

and obtain

$$
\begin{aligned}
& I_{20}=\frac{1}{1-\epsilon} \int_{0}^{1} d y\left[y^{\epsilon / 2} Q^{-2-\epsilon}\left(1+\xi y-\xi^{2} y^{2}-\xi^{3} y^{3}\right)+y^{1-\epsilon / 2} L^{-2-\epsilon}(1+\xi)^{3}\right], \\
& I_{10}=-\frac{1}{\epsilon} \int_{0}^{1} d y\left[y^{\epsilon / 2} Q^{-2-\epsilon}\left(1-\xi^{2} y^{2}\right)+y^{-\epsilon / 2} L^{-2-\epsilon}(1+\xi)^{2}\right], \\
& I_{01}=\frac{1}{1-\epsilon} \int_{0}^{1} d y\left\{y^{1+\epsilon / 2} Q^{-2-\epsilon}(1-\xi y)+y^{-\epsilon / 2} L^{-2-\epsilon}(1+\xi)\right],
\end{aligned}
$$

with $\xi=X-1$ and where

$$
Q=(1+\xi y)^{2}+\eta y, \quad L=\eta+X^{2} y .
$$

The relevant combination of integrals is

$$
\mathcal{I}=I_{20}-I_{10}-\rho I_{01} .
$$

Collecting the various terms we obtain

$$
\begin{aligned}
\mathcal{S}_{221} & =\int_{0}^{1} d y\left\{\frac{1}{\epsilon}\left[y^{\epsilon / 2}\left(1-\xi^{2} y^{2}\right) Q^{-2-\epsilon}+y^{-\epsilon / 2} X^{2} L^{-2-\epsilon}\right]+\frac{1}{1-\epsilon}\right. \\
& \left.\times\left[y^{\epsilon / 2}\left(1+(\xi-\rho) y-(\xi-\rho) \xi y^{2}-\xi^{3} y^{3}\right) Q^{-2-\epsilon}+y^{-\epsilon / 2}\left(X^{2} y-\rho\right) X L^{-2-\epsilon}\right]\right\} .
\end{aligned}
$$

From its definition, Eq.(345), we see that $\eta=0$ for $x_{1}=1$ and $x_{2}=0$. While the quadratic form in $y$ contains a constant term, the linear form $\eta+X^{2} y$ vanishes if the parameters $\left\{x_{1}^{\prime}=1-x_{1}, x_{2}, y\right\}$ are set to zero. This is the origin of the overlapping divergence that we must disentangle. First of all we change variable in order to factorize the divergent terms: $x_{1}=1-x_{1}^{\prime}$ and,successively, $x_{2}=x_{1} x_{2}^{\prime}$, so that divergences will arise from the behavior of the integrands at the point $x_{1}^{\prime}=0$. After the transformation we get

$$
\xi=-\frac{x_{1}}{1-x_{1} x_{2}}, \quad \eta=x_{1} \frac{\left(1+x_{2}\right)^{2}}{x_{2}\left(1-x_{1} x_{2}\right)} .
$$


When we exchange the $x_{1}-x_{2}$ integrations the limits become $0 \leq x_{2} \leq+\infty$ and $0 \leq x_{1} \leq$ $1 /\left(1+x_{2}\right)$. We change again variable, $x_{1}=x_{1}^{\prime} /\left(1+x_{2}\right)$, so that $0 \leq x_{1}^{\prime} \leq 1$ and

$$
\xi=-\frac{x_{1}}{1+x_{2}-x_{1} x_{2}}, \quad \eta=x_{1} \frac{\left(1+x_{2}\right)^{2}}{x_{2}\left(1+x_{2}-x_{1} x_{2}\right)} .
$$

\subsubsection{Defoliation of IR divergent integrals}

At this point we introduce a simple example to explain the method. Consider

$$
I=\int_{0}^{1} d x \int_{0}^{1} d y Q^{-2-\epsilon}, \quad Q=x(1+x)+y^{2} .
$$

A minimal set of zeros is a minimal set of parameter values that satisfy $Q=0$. In our simple case we have $\{x=y=0\}$. A defoliation is the procedure that allows for a factorization of the divergent part (in the limit $\epsilon \rightarrow 0$ ) through different steps. In our case,

$$
I=\left\{\int_{0}^{1} d x \int_{0}^{x} d y+\int_{0}^{1} d y \int_{0}^{y} d x\right\} Q^{-2-\epsilon}=\int_{0}^{1} d x \int_{0}^{1} d y\left[x^{-1-\epsilon} Q_{\{x=0\}}^{-2-\epsilon}+y^{-1-\epsilon} Q_{\{y=0\}}^{-2-\epsilon}\right\}
$$

where the defoliated Q's are:

$$
Q_{\{x=0\}}=1+x+x y^{2}, \quad Q_{\{y=0\}}=x(1+x y)+y .
$$

Since the first form never vanishes over the whole parametric space, including the border, the procedure stops here for the first integral, while for the second one has to iterate until the condition is met. To give an example, if we start with $Q=x(1-x)+y^{2}$ the first defoliation gives $x(1-x y)+y$ but also $1-x+x y^{2}$ which has a set of zeros at $\{x=1, y=0\}$; then one has to transform, $x=1-x^{\prime}$, and iterate.

Note that the algorithm is perfectly defined for all integrands that may vanish only at the boundaries of the parametric space. $S_{p}^{221}$ is the first example of a strategy which differs considerably from the minimal BT approach. In the latter the main attempt is toward raising powers in the integrand while here the powers are untouched and the whole idea is about factorizing the singular behavior into simple factors that can be integrated analytically, leaving non singular terms to be integrated numerically.

Other cases, where the Feynman integrand is not semi-positive defined (presence of thresholds) are more difficult to analyze. However this never happens for two-point functions and their derivatives. Infrared singularities are finally extracted by using Taylor expansion, typically

$$
\int_{0}^{1} d x \int_{0}^{1} d y x^{-1-\epsilon} f(x, y, \epsilon)=-\frac{1}{\epsilon} \int_{0}^{1} d y f(0, y, \epsilon)+\int_{0}^{1} d x \int_{0}^{1} d y\left(\frac{f(x, y, \epsilon)}{x^{1+\epsilon}}\right)_{+} .
$$

Note that only now one can expand around $\epsilon=0$. Various branches in the procedure are not

uniquely defined and one is led to the search of the path which brings the minimal number of iterations. 


\subsection{2 $S_{p}^{221}$ method I}

After these preliminar considerations we go back to Eq.(352). After all transformations we rewrite $Q$ and $L$ obtaining

$$
\begin{aligned}
Q & =\left(1+x_{2}-x_{1} x_{2}\right)^{2} x_{2}+\left(1+x_{2}^{2}\right)\left(1+x_{2}-x_{1} x_{2}\right) x_{1} y+x_{1}^{2} x_{2} y^{2} \\
L & =\left(1+x_{2}\right)^{2}\left(1+x_{2}-x_{1} x_{2}\right) x_{1}+\left(1+x_{2}\right)^{2}\left(1-x_{1}\right)^{2} x_{2} y
\end{aligned}
$$

The integration range is from 0 to 1 for $x_{1}$ and $y$ and from 0 to $\infty$ for $x_{2}$. It is easily seen that $Q$ and $L$ may vanish only for zero values of the parameters. In order to extract the divergences we use the relation:

$$
\begin{aligned}
\int_{0}^{1} d x \int_{0}^{1} d y f(x, y) & =\int_{0}^{1} d x \int_{0}^{x} d y f(x, y)+\int_{0}^{1} d y \int_{0}^{y} d x f(x, y) \\
& =\int_{0}^{1} d x \int_{0}^{1} d y(x f(x, x y)+y f(x y, y))
\end{aligned}
$$

Since our functions $f=Q, L$ are polynomials to a power $-2-\epsilon$, we have $f(x, x y)=x^{-2-\epsilon} g(x, y)$, where $g$ has a zero at $x=0$ of lower order. We split the integration over $x_{2}$ in two parts, the first in the interval $[0,1]$ and the second in $[1, \infty]$. In the former we iterate the procedure until we reach polynomials that do not vanish inside the parametric space. In the latter we perform the procedure only for $x_{1}$ and $y$.

In the limit $x_{2} \rightarrow \infty$ some of the integrals may lead to a divergency because of the presence of divergent factors, other than in $Q$ and $L$. In that case we perform the transformation $x_{2} \rightarrow 1 / x_{2}$ and iterate the procedure for $x_{2} \in[0,1]$. At the end we obtain integrands where the divergences are contained only in the following factors:

$$
x_{1}^{-1-\epsilon}, \quad x_{1}^{-1-2 \epsilon}, \quad x_{2}^{-1-\epsilon}, \quad \text { or } \quad y^{-1-2 \epsilon} .
$$

The final answer is obtained by using the following relation:

$$
\int_{0}^{1} d y y^{-1-2 \epsilon} f(y)=\int_{0}^{1} d y y^{-2 \epsilon}\left(\frac{f(y)}{y}\right)_{+}-\frac{1}{2 \epsilon} f(0) .
$$

In this way, after the expansion around $\epsilon=0$ we obtain

$$
\int_{0}^{1} d x_{1} \int_{0}^{x_{1}} d x_{2}\left[x_{2}\left(1-x_{2}\right)\right]^{-1-\epsilon / 2} \mathcal{S}_{221}=\frac{1}{\epsilon^{2}} \mathcal{S}_{221}^{2}+\frac{1}{\epsilon} \mathcal{S}_{221}^{1}+\mathcal{S}_{221}^{0}
$$

where $\mathcal{S}_{221}^{2}, \mathcal{S}_{221}^{1}$ and $\mathcal{S}_{221}^{0}$ are expressed in terms of smooth integrands. Furthermore, $\mathcal{S}_{221}^{2}$ is the sum of integrals which can be calculated analytically:

$$
\begin{aligned}
\mathcal{S}_{221}^{2} & =-\frac{1}{2} \int_{0}^{\infty} d x_{2} \int_{0}^{1} d y \frac{x_{2}}{1+x_{2}}\left(1+x_{2}+x_{2} y\right)^{-2} \\
& -\frac{1}{2} \int_{1}^{\infty} d x_{2} \int_{0}^{1} d x_{1} \frac{x_{2}}{1+x_{2}}\left(x_{1}+x_{2}+x_{1} x_{2}\right)^{-2}
\end{aligned}
$$




$$
\begin{aligned}
& -\frac{1}{2} \int_{0}^{1} d x_{1} \int_{0}^{1} d x_{2} \frac{1}{1+x_{2}}\left(1+x_{2}+x_{1} x_{2}\right)^{-2}-\frac{1}{2} \int_{0}^{1} d x_{1}\left(1+x_{1}\right)^{-2} \\
& -\frac{1}{2} \int_{0}^{1} d x_{1} \int_{0}^{1} d x_{2} \frac{x_{2}}{1+x_{1} x_{2}}\left(1+x_{2}+x_{1} x_{2}\right)^{-2}-\frac{1}{2} \int_{0}^{1} d x_{2}\left(1+x_{2}\right)^{-2} \\
& =\frac{1}{2}(\ln 2-1)-\frac{1}{2}\left(\ln 2-\ln \frac{3}{2}\right)+\frac{1}{2}\left(\ln 3-2 \ln 2-\frac{1}{2}\right) \\
& +\frac{1}{2}\left(3 \ln 2-2 \ln 3-\frac{1}{2}\right)=-1 .
\end{aligned}
$$

For the single IR pole we obtain a relatively long expression that will be reported in Appendix D. The expression of $\mathcal{S}_{221}^{0}$ is too long and of little interest from the analytical point of view. Therefore it will not be reported here.

\subsection{3 $S_{p}^{221}$ method II}

To check the correctness of method I we have also used a different strategy. In Eq.(346) after changing variables as described after Eq.(352) we exchange the order of integration, so that $x_{2} \in$ $[0, \infty]$ and $x_{1} \in\left[0,1 /\left(1+x_{2}\right)\right]$. Two other changes of variables will bring all integrals in the interval $[0,1]$ : they are $x_{1}=x_{1}^{\prime} /\left(1+x_{2}\right)$ and $x_{2}=1 / x_{2}^{\prime}-1$. After rationalization we end up with two polynomials to the power $-2-\epsilon$. They are

$$
\begin{aligned}
Q^{t} & =y x_{1}\left(1-x_{1} x_{2}\right)\left[x_{2}^{2}+\left(1-x_{2}\right)^{2}\right]+y^{2} x_{1}^{2} x_{2}\left(1-x_{2}\right)^{2}+\left(1-x_{1} x_{2}\right)^{2} x 2, \\
L^{t} & =y x_{1}^{2} x_{2}\left(1-x_{2}\right)^{2}+y x_{2}\left(1-x_{1} x_{2}\right)^{2}+x_{1}\left(1-x_{1} x_{2}\right)\left[2 x_{2}\left(1-x_{2}\right)(1-y)+x_{2}^{2}+\left(1-x_{2}\right)^{2}\right] .
\end{aligned}
$$

We have written the two polynomials in a form that makes clear their semi-positive definiteness over the cube $0 \leq\left\{x_{1}, x_{2}, y\right\} \leq 1$. For more than two variables, $x_{1} \ldots x_{n}$, defoliation is based on inserting the following identity,

$$
1=\sum_{l=1}^{n} \prod_{j \neq l=1}^{n} \theta\left(x_{l} \geq x_{j}\right),
$$

in the integral. Several defoliations are needed and we summarize the results in the following list. First $Q^{t}$,

$$
\begin{array}{rlll}
\left\{x_{1}=x_{2}=0\right\} & : & Q^{t} \rightarrow Q_{1}^{t}+Q_{2}^{t}, \\
\left\{x_{1}=x_{2}=1\right\} & : & Q_{1}^{t} \rightarrow Q_{11}^{t}+Q_{12}^{t}, \\
\left\{y=x_{2}=0\right\} & : & Q_{11}^{t} \rightarrow Q_{111}^{t}+Q_{112}^{t}, \\
\left\{y=x_{1}=0\right\} & : & Q_{12}^{t} \rightarrow Q_{121}^{t}+Q_{122}^{t}, \\
\left\{y=x_{2}=0\right\} & : & Q_{2}^{t} \rightarrow Q_{21}^{t}+Q_{22}^{t}, \\
\left\{y=x_{1}=x_{2}=1\right\} & : & Q_{21}^{t} \rightarrow Q_{211}^{t}+Q_{212}^{t}+Q_{213}^{t}, \\
\left\{x_{1}=x_{2}=1\right\} & : & Q_{22}^{t} \rightarrow Q_{221}^{t}+Q_{222}^{t},
\end{array}
$$




$$
\begin{array}{lll}
\left\{y=x_{2}=0\right\} & : \quad Q_{221}^{t} \rightarrow Q_{2211}^{t}+Q_{2212}^{t} \\
\left\{y=x_{1}=0\right\} & : \quad Q_{222}^{t} \rightarrow Q_{2221}^{t}+Q_{2222}^{t}
\end{array}
$$

and then $L^{t}$,

$$
\begin{array}{rll}
\left\{y=x_{1}=0\right\} & : & L^{t} \rightarrow L_{1}^{t}+L_{2}^{t}, \\
\left\{x_{1}=x_{2}=0\right\} & : & L_{1}^{t} \rightarrow L_{11}^{t}+L_{12}^{t}, \\
\left\{y=x_{1}=x_{2}=1\right\} & : & L_{11}^{t} \rightarrow L_{111}^{t}+L_{112}^{t}+L_{113}^{t}, \\
\left\{y=x_{1}=x_{2}=1\right\} & : & L_{12}^{t} \rightarrow L_{121}^{t}+L_{122}^{t}+L_{123}^{t}, \\
\left\{x_{1}=x_{2}=1\right\} & : & L_{2}^{t} \rightarrow L_{21}^{t}+L_{22}^{t} .
\end{array}
$$

In the above procedure it is understood that the appropriate change of variables is performed to bring the zeros at the origin at each step. At the end of any chain all polynomials are strictly positive defined in the unit cube. One final step is needed to disentangle integrals of the following form:

$$
J(a, b)=\int_{0}^{1} d x \int_{0}^{1} d y(1-x)^{-1-a \epsilon}(1-x y)^{-1-b \epsilon} f(x, y),
$$

where $f(x, y)$ is analytic in the integration domain. We obtain

$$
\begin{aligned}
J(a, b) & =\int_{0}^{1} d x \int_{0}^{1} d y\left[x^{-1-(a+b) \epsilon}(1+y-x y)^{-1-b \epsilon} f(1-x, 1-x y)\right. \\
& +x^{-1-a \epsilon} y^{-1-(a+b) \epsilon}(1+x-x y)^{-1-b \epsilon} f(1-x y, 1-y) .
\end{aligned}
$$

Finally all divergent integrals factorize, an example being as follows:

$$
\begin{gathered}
K(a, b)=\int_{0}^{1} d x \int_{0}^{1} d y x^{-1-a \epsilon} y^{-1-b \epsilon} F(x, y) \\
K(a, b)=\frac{1}{a b \epsilon^{2}} F(0,0)-\frac{1}{a \epsilon} \int_{0}^{1} d y\left(\frac{F(0, y)}{y^{1+b \epsilon}}\right)_{+}-\frac{1}{b \epsilon} \int_{0}^{1} d x\left(\frac{F(x, 0)}{x^{1+a \epsilon}}\right)_{+} \\
+\int_{0}^{1} d x \int_{0}^{1} d y\left(\frac{F(x, y)}{x^{1+a \epsilon} y^{1+b \epsilon}}\right)_{++}
\end{gathered}
$$

where we have introduced $\mathrm{a}^{\prime} t^{\prime}$ and $\mathrm{a}^{\prime}++^{\prime}$ distribution

$$
\begin{aligned}
\int_{0}^{1} d x\left(\frac{g(x)}{x^{1+a \epsilon}}\right)_{+} & =\int_{0}^{1} d x \frac{g(x)-g(0)}{x}\left[1-a \epsilon \ln x+\mathcal{O}\left(\epsilon^{2}\right)\right], \\
\int_{0}^{1} d x \int_{0}^{1} d y\left(\frac{h(x, y)}{x^{1+a \epsilon} y^{1+b \epsilon}}\right)_{++} & =\int_{0}^{1} d x \int_{0}^{1} d y \frac{h(x, y)-h(0, y)-h(x, 0)+h(0,0)}{x y} \\
& \times\left[1-\epsilon(a \ln x+b \ln y)+\mathcal{O}\left(\epsilon^{2}\right)\right] .
\end{aligned}
$$

Also in this case the residue of the double IR pole can be computed analytically and gives -1 . The explicit expressions for the residue of the single IR pole, as well as for the finite part will not be reported. 


\subsubsection{Another configuration for $S_{p}^{221}$}

There is another configuration where Eq.(288) is satisfied,

$$
\text { b) } m_{1}=m_{2}=m_{4}=m_{5}=m, m_{3}=0 \quad \text { and } \quad s=0 \text {. }
$$

Actually this configuration gives a nice example of a necessary condition which, however, is not sufficient. Let us consider the corresponding derivative,

$$
\begin{aligned}
S_{p}^{221 \mid b} & =\frac{\partial}{\partial p^{2}} S^{221 \mid b}=-\left(\frac{\mu^{2}}{\pi}\right)^{\epsilon} \Gamma(2+\epsilon) \int_{0}^{1} d x_{1} \int_{0}^{x_{1}} d x_{2}\left[x_{2}\left(1-x_{2}\right)\right]^{-1-\epsilon / 2} \\
& \times \int_{0}^{1} d y \int_{X y}^{1+(X-1) y} d z y^{\epsilon / 2}[z(z-1)-\rho y] U_{221 \mid b}^{-2-\epsilon},
\end{aligned}
$$

where the quadratic form $U_{221 \mid b}$ is defined by

$$
U_{221 \mid b}=-p^{2} z^{2}+p^{2} z+\left(m_{x x}^{2}-m^{2}-X p^{2}\right) y+m^{2},
$$

and $m_{x x}^{2}$ is given in Eq.(281). Next we define the $p^{2} \rightarrow 0$ limit of $U_{221 \mid b}$,

$$
U_{221 \mid b}\left(p^{2}=0\right)=\frac{m^{2}}{x_{2}} \chi_{221|b| \mathrm{os}}, \quad \chi_{221|b| \mathrm{os}}=\left(1-x_{2}\right) y+x_{2} .
$$

Although the point $y=x_{2}=0$ is the candidate for generating an infrared divergence we can

prove, by direct calculation, that the result is infrared finite. When we set $\epsilon=0$ it is easy to see that the $y, z$ integrations are trivial and

$$
\mathcal{S}_{221 \mid b}=\int_{0}^{1} d x_{1} \int_{0}^{x_{1}} d x_{2} \mathcal{F}_{221 \mid b}, \quad \mathcal{F}_{221 \mid b}=\sum_{n=2}^{6} \frac{A_{n}\left(x_{1}\right)}{\left(1-x_{2}\right)^{n}}+\sum_{n=3}^{7} \frac{B_{n}\left(x_{1}\right)}{\left(1-x_{2}\right)^{n}} \ln x_{2},
$$

where the various coefficients are:

$$
\begin{aligned}
& A_{6}=3-6 x_{1}+3 x_{1}^{2}, \quad A_{5}=-\frac{17}{2}+14 x_{1}-\frac{11}{2} x_{1}^{2}, \quad A_{4}=7-\frac{13}{2} x_{1}+\frac{1}{2} x_{1}^{2}, \\
& A_{3}=-2+\frac{1}{2} x_{1}, \quad A_{2}=\frac{1}{3}, \quad B_{7}=3-6 x_{1}+3 x_{1}^{2}, \\
& B_{6}=-10+17 x_{1}-7 x_{1}^{2}, \quad B_{5}=11-13 x_{1}+3 x_{1}^{2} \quad B_{4}=-5+3 x_{1}, \quad B_{3}=1 .
\end{aligned}
$$

The non trivial part of the $x_{2}$-integration is performed recursively by using the following relation:

$$
\begin{aligned}
J_{-n} & =\int_{0}^{x_{1}} d x_{2} \frac{\ln x_{2}}{\left(1-x_{2}\right)^{n}}, \quad J_{-2}=\ln \left(1-x_{1}\right)-\frac{x_{1}}{x_{1}-1} \ln x_{1} . \\
n J_{-(n+1)} & =(n-1) J_{-n}+\frac{1}{n-1}-\frac{1}{n-1} \frac{1}{\left(1-x_{1}\right)^{n-1}}+\frac{x_{1} \ln x_{1}}{\left(1-x_{1}\right)^{n}}
\end{aligned}
$$


Finally the $x_{1}$-integration uses the following results 9 :

$$
\begin{aligned}
& \int_{0}^{1} d x x^{-4} \ln (1-x)=-\int_{0}^{1} d x\left(x^{-3}+\frac{1}{2} x^{-2}+\frac{1}{3} x^{-1}\right)-\frac{11}{18} \\
& \int_{0}^{1} d x x^{-3} \ln (1-x)=-\int_{0}^{1} d x\left(x^{-2}+\frac{1}{2} x^{-1}\right)-\frac{3}{4} \\
& \int_{0}^{1} d x x^{-2} \ln (1-x)=-\int_{0}^{1} d x x^{-1}-1, \\
& \int_{0}^{1} d x x^{-1} \ln (1-x)=-\zeta(2) \\
&\left.\int_{0}^{1} d x x_{1}^{n} \ln x=-\frac{1}{(n+1)^{2}}, \quad \int_{0}^{1} d x x_{1}^{n} \ln (1-x)\right)=-\frac{1}{n+1} \sum_{l=1}^{n+1} \frac{1}{l} .
\end{aligned}
$$

The final result is

$$
\mathcal{S}_{221 \mid b}=-\frac{13}{72}
$$

proving that the b-configuration is infrared finite.

\subsection{Tensor integrals of the $S^{221}$ family}

The scalar $S^{221}$ integral has been computed at $n=4$ and the absence of double-logarithms has shown some advantage for the integration in the complex plane. On the contrary, tensor integrals of the same family are ultraviolet divergent and this feature seems lost. However, this problem is encompassed by the procedure of scalarization. Scalarization can never be complete, in contrast with the one-loop case [21], and we will stop the procedure whenever an irreducible numerator is encountered. A tensor integral will be irreducible when the numerator cannot be expressed as a linear combination of those propagators which are present at that particular moment of the reduction. In a sense we scalarize the obvious and explicitly compute the rest.

Let us define the following function:

$$
\pi^{4} S_{5}^{2-i / 2,1,2-j / 2}\left(\mu_{1} \ldots \mu_{i} \mid \nu_{1} \ldots \nu_{j}\right)=\mu^{2 \epsilon} \int d^{n} q_{1} d^{n} q_{2} \frac{q_{1}^{\mu_{1}} \ldots q_{1}^{\mu_{i}} q_{2}^{\nu_{1}} \ldots q_{2}^{\nu_{j}}}{\left(q_{1}^{2}+m_{1}^{2}\right) \ldots\left(\left(q_{2}+p\right)^{2}+m_{5}^{2}\right)}
$$

where we assume $i+j \leq 3$ and where $\alpha$ and $\beta$ have been changed in order to account for the effective degree of convergence: for $i+j \geq 2$ the function is overall divergent, for $i \geq 2$ the $\alpha \gamma$ sub-diagram is divergent, etc. Note that we always indicate the total number of internal lines.

Let us consider some example,

$$
S_{5}^{1 / 2,1,2}(p, p, p \mid 0),
$$

\footnotetext{
${ }^{2}$ For the first equation we disagree with the result of p. 20, ref. 30
} 
and perform the reduction. Since there are enough propagators, the reduction is particularly simple,

$$
\begin{aligned}
q_{1} \cdot p & =\frac{1}{2}\left\{\left[\left(q_{1}+p\right)^{2}+m_{2}^{2}\right]-\left[q_{1}^{2}+m_{1}^{2}\right]+m_{1}^{2}-m_{2}^{2}-p^{2}\right\}, \\
q_{2} \cdot p & =\frac{1}{2}\left\{\left[\left(q_{2}+p\right)^{2}+m_{5}^{2}\right]-\left[q_{2}^{2}+m_{4}^{2}\right]+m_{4}^{2}-m_{5}^{2}-p^{2}\right\} . \\
q_{1} \cdot q_{2} & =-\frac{1}{2}\left\{\left[\left(q_{1}-q_{2}\right)^{2}+m_{3}^{2}\right]-\left[q_{1}^{2}+m_{1}^{2}\right]-\left[q_{2}^{2}+m_{4}^{2}\right]-m_{3}^{2}+m_{1}^{2}+m_{4}^{2}\right\},
\end{aligned}
$$

etc. and give the following result:

$$
\begin{aligned}
8 S_{5}^{1 / 2,1,2}(p, p, p \mid 0) & =\left(m_{1}^{2}-m_{2}^{2}-p^{2}\right)^{3} S^{221}(0 \mid 0) \\
& +\left(m_{1}^{2}-m_{2}^{2}-p^{2}\right)^{2} S_{4}^{121}\left(0|0| p, m_{1}, m_{3}, m_{4}, m_{5}\right) \\
& +\left[4 p^{2}\left(m_{1}^{2}-m_{2}^{2}\right)-7 p^{4}-\left(m_{1}^{2}-m_{2}^{2}\right)^{2}\right] S_{4}^{121}\left(0|0|-p, m_{2}, m_{3}, m_{5}, m_{4}\right) \\
& +2\left(m_{1}^{2}-m_{2}^{2}-p^{2}\right) S_{4}^{1 / 2,2,1}\left(p|0| p, m_{1}, m_{3}, m_{4}, m_{5}\right) \\
& +2\left(5 p^{2}-m_{1}^{2}+m_{2}^{2}\right) S_{4}^{1 / 2,2,1}\left(p|0|-p, m_{2}, m_{3}, m_{5}, m_{4}\right) \\
& +4\left[S_{4}^{021}\left(p, p|0| p, m_{1}, m_{3}, m_{4}, m_{5}\right)-S_{4}^{021}\left(p, p|0|-p, m_{2}, m_{3}, m_{5}, m_{4}\right)\right]
\end{aligned}
$$

Another interesting example of scalarization is represented by

$$
\begin{aligned}
8 S_{5}^{1,1,3 / 2}(p, p \mid p) & =-p_{12}^{4} p_{45}^{2} S_{5}^{221}(0 \mid 0)+p_{12}^{2} p_{45}^{2} S_{4}^{121}\left(0|0| p, m_{1}, m_{3}, m_{4}, m_{5}\right) \\
& -p_{45}^{2}\left(p_{12}^{2}+2 p^{2}\right) S_{4}^{121}\left(0|0|-p, m_{2}, m_{3}, m_{5}, m_{4}\right) \\
& +p_{12}^{2}\left[S_{4}^{121}\left(0|0| p, m_{4}, m_{3}, m_{1}, m_{2}\right)-S_{5}^{121}\left(0|0|-p, m_{5}, m_{3}, m_{2}, m_{1}\right)\right] \\
& +2 p_{45}^{2}\left[S_{4}^{1 / 2,2,1}\left(p|0|-p, m_{2}, m_{3}, m_{5}, m_{4}\right)-S_{4}^{1 / 2,2,1}\left(p|0| p, m_{1}, m_{3}, m_{4}, m_{5}\right)\right] \\
& +\left(p^{2}+m_{2}^{2}+p_{12}^{2}\right) S_{3}^{111}\left(0|0|-p, m_{2}, m_{3}, m_{4}\right)+p_{12}^{2}\left[S_{3}^{111}\left(0|0| p, m_{1}, m_{3}, m_{5}\right)\right. \\
& \left.-S_{3}^{111}\left(0|0| 0, m_{1}, m_{3}, m_{4}\right)\right]+\left(p^{2}-m_{2}^{2}\right) S_{3}^{111}\left(0|0|-p, m_{2}, m_{3}, m_{4}\right) \\
& -\left(p_{12}^{2}+2 p^{2}\right) S_{3}^{111}\left(0|0| 0, m_{2}, m_{3}, m_{5}\right)-2\left[S_{3}^{1 / 2,1,1}\left(p|0| p, m_{1}, m_{3}, m_{5}\right)\right. \\
& \left.+S_{3}^{1 / 2,1,1}\left(p|0|-p, m_{2}, m_{3}, m_{4}\right)\right]
\end{aligned}
$$

where we have introduced the following combinations: $p_{i j}^{2}=p^{2}-m_{i}^{2}+m_{j}^{2}$. Other relevant examples can be obtained by permutation of masses and all of them indicate that we need at most the scalar $S_{5}$-function plus a combination of tensor integrals with a number of internal legs which is $\leq 4$.

\subsection{A realistic example}

To discuss some realistic example of tensorial reduction in the $S_{5}$-family let us consider one of the two-loop diagrams that in QED contribute to the photon self-energy, see Fig. 19. We derive 


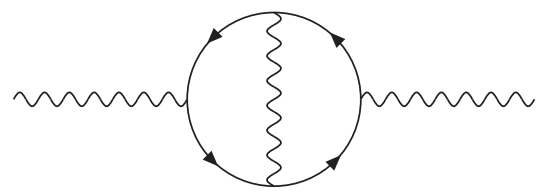

Figure 19: A contribution to the photon self-energy in QED

the following expression for the vacuum polarization:

$$
\Pi_{\mu \nu}^{(2)}=\pi^{4} e^{4} \Pi_{0}^{(2)}\left(\delta_{\mu \nu}-\frac{p_{\mu} p_{\nu}}{p^{2}}\right), \quad \Pi_{0}^{(2)}=\frac{1}{3-\epsilon} S .
$$

For the function $S$ we simplify the numerator and obtain

$$
\begin{aligned}
S & =4\left[(\epsilon-2) p^{4}+8 m^{4}+2 \epsilon p^{2} m^{2}\right] S^{221}\left(p^{2} ; m, m, 0, m, m\right) \\
& +2\left[8 m^{2}-\left(8-2 \epsilon-\epsilon^{2}\right) p^{2}\right] B_{0}^{2}\left(p^{2} ; m, m\right)+8(2-\epsilon) p^{2} B_{1}^{2}\left(p^{2} ; m, m\right) \\
& -16\left[2 m^{2}+(\epsilon-2) p^{2}\right] S_{4}^{121}\left(p^{2} ; m, 0, m, m\right)+4 \epsilon(2-\epsilon) S^{111}\left(p^{2} ; m, 0, m\right) \\
& +8(\epsilon-2) S^{111}(0 ; m, 0, m)+8(2-\epsilon) A_{0}(m) B_{0}\left(p^{2} ; m, m\right) .
\end{aligned}
$$

Note that $S$ is ultraviolet divergent and must be associated with the corresponding subtraction diagrams of Fig. 20.
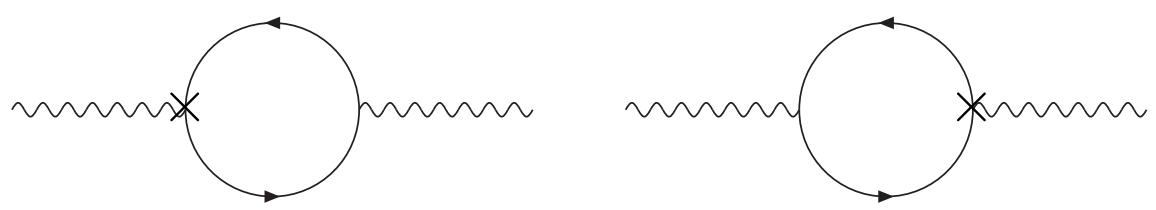

Figure 20: The one-loop subtraction diagrams, containing a one-loop counter-term (represented by a $\times$ ), associated to the two-loop diagram of Fig. 19.

\section{Numerical Results}

The whole set of algorithms presented in the previous sections has been translated into a FORM code [22] and the output has been used to create a FORTRAN code.

In this section we will present numerical results for two-loop two-point functions (self-energies), based on the results developed in the previous sections. In the following we present a detailed 
comparison among different formulations for the same topology, different integration routines and, for few special cases, between our numerical results and the available analytical calculations. Typically we have used three different sub-routines for numerical integration: D01EAF, a multidimensional adaptive quadrature over hyper-rectangle, multiple integrands; D01GBF, a multidimensional quadrature over hyper-rectangle, Monte Carlo method; D01GDF, a multi-dimensional quadrature, general product region, number-theoretic method.

\subsection{Numerical results for $S^{121}$}

To check our results for this topology we consider two special cases, namely a) $m_{1}=m, m_{i}=0$ for $i \geq 2$ and b) $m_{2}=m_{4}=m, m_{1}=m_{3}=0$ which correspond to Eq. (38) and to Eq.(45) of ref. [26]. Results and comparisons for configuration b) are shown in Tab. 11. The first entry is based on method II, Eq.(79), and the NAG [23] subroutine D01EAF; the second entry refers to the same Eq. (79) but uses the NAG subroutine D01GBF. The latter usually returns a smaller, underestimated,integration error. The former has a larger error, typically $0.06 \%$ for the real part and $1.7 \%(0.9 \%, 0.5 \%)$ for the imaginary part at $81 \mathrm{GeV}(83 \mathrm{GeV}, 85 \mathrm{GeV})$. Relative deviations with the analytical results are $\leq 0.006 \%$ for the real part and $0.09 \%(0.05 \%, 0.04 \%)$ for the imaginary part $(0.07 \%, 0.02 \%, 0.01 \%$ for D01GBF). As expected the largest errors occur around threshold.

For configuration a) we report results and comparisons in Tab. 2. In Appendix E we give the analytical evaluation of the imaginary part of $S^{121}$ which receives a contribution from a two-particle cut and a three-particle cut. Comparisons with the numerical result are shown in Tabs. 3 4. The relative deviation with respect to the analytical result is $\leq 0.04 \%$ over a wide range of energies, below and above the three-particle cut. In Tab. 5 we scan the threshold regions, $\sqrt{s}=m_{3}+m_{4}$ and $\sqrt{s}=m_{1}+m_{2}+m_{4}$. Even at one $\mathrm{MeV}$ above threshold we are able to reproduce the imaginary part with a relative precision of less than $1 \%$

\subsection{Numerical results for $S^{131}$}

The interesting case for $S^{131}$ is represented by $m_{3}=m_{5}$. No analytical results are usually shown in the literature since all authors write

$$
S^{131}\left(p^{2} ; m_{1}, m_{2}, m_{3}, m_{4}, m_{3}\right)=-\frac{\partial}{\partial m_{3}^{2}} S^{121}\left(p^{2} ; m_{1}, m_{2}, m_{3}, m_{4}\right) .
$$

We have done the same and compared internally $S^{131}$ with the numerical derivative of $S^{121}$. However, numerical differentiation of functions that are already the result of numerical integration enjoy poor reputation, a fact that we confirm. Unless a extremely accurate but time consuming evaluation of the multi-dimensional integral is performed we end up with large uncertainties in the derivative. Fortunately we can still check our results, at least for the imaginary part where an analytical approach is available. The results are shown in Tab. 6 and the larger error in the derivative, for larger energies, is due to the three-particle cut. The behavior of $S^{131}$ for $m_{5}=m_{3}$

and around the normal threshold $\sqrt{s}=m_{3}+m_{4}$ is shown in Fig. 21 and also reported in Tab. 7 where we see that numerical precision is worst on the high energy side of the threshold, where 
the imaginary part in non-zero. However, it is enough to go $100 \mathrm{MeV}$ away from threshold to be better that $0.1 \%(0.3 \%$ at $50 \mathrm{MeV})$.

\subsection{Numerical results for $S^{221}$}

For $S^{221}$ we have compared our results with those of ref. 27] where the authors have introduced a one-dimensional integral representation of $S^{221}$. In particular we refer to Tab. 1 of ref. [27]. Comparisons are shown in Tab. 8 where we have selected their numerical results with the smaller error. In this case we use the NAG subroutine D01GDF which calculates an approximation to the integral using the Korobov-Conroy number theoretic method with a Monte-Carlo error estimate; our two entries in Tab. 8 refer to the choice of 10193 - 101 and $22807 \cdot 151$ points respectively. Note that with their choice of masses, $m_{n}=\sqrt{n} \mathrm{GeV}$, the normal thresholds are at $2.414 \mathrm{GeV}$, $4.236 \mathrm{GeV}$ (two-particle cuts) and $4.968 \mathrm{GeV}, 5.146 \mathrm{GeV}$ (three-particle cuts) respectively. Only the last energy is above all normal thresholds. There are two configurations where $S^{221}$ can be computed using the appropriate expansion: let variables $x, y$ be defined as in Eq.(421), we can give the $|y| \ll 1$ and the $|x| \ll 1$ expansion of $S^{221}(s ; m, 0,0,0,0)$, as discussed in Appendix F. Results are shown in Tab. 9. By using Eq.(424) we can compare the low- $s$ behavior of $S^{221}(s ; m, m, m, 0,0)$ which is shown in Tab. 10.

\section{Conclusions}

For one-loop diagrams the analytical way has been fully successful, resulting in compact expressions containing at most di-logarithms For multi-loop diagrams, special cases have been equal successful, see for instance ref. [29] and ref. [30], resulting in expressions containing generalized Nielsen poly-logarithms [31]. However, this approach can hardly been extended beyond one-loop for general massive diagrams. For instance, the work of ref. [26] and [27] already shows that for two-loop integrals which contain a massive three-particle cut, the results in general cannot be expressed in terms of generalized poly-logarithms. Sometimes special cases of multi-loop diagrams can be expressed with the help of generalized hypergeometric functions or of other higher transcendental functions, but in the end this is equivalent to some series representation that always has a restricted region of convergence. In other words, from a practical point of view, they can hardly be extended above the closest normal or pseudo-threshold.

This paper represents the second in a series devoted to numerical evaluation of multi-loop Feynman diagrams with the declared intent of covering all regions in the external parameters. In the first paper [7] we have introduced the minimal Bernstein-Tkachov algorithm and discussed the simplest two-loop topology, $S^{111}$ or sunset diagram.

In this paper we extended the previous work to cover all two-loop two-point functions, i.e. the self-energies. Each topology has been written with an integral representation in the Feynman parametric space which is particularly suited for numerical integration. For two external legs it turns out that distortion of the integration hyper-contour can be avoided. 


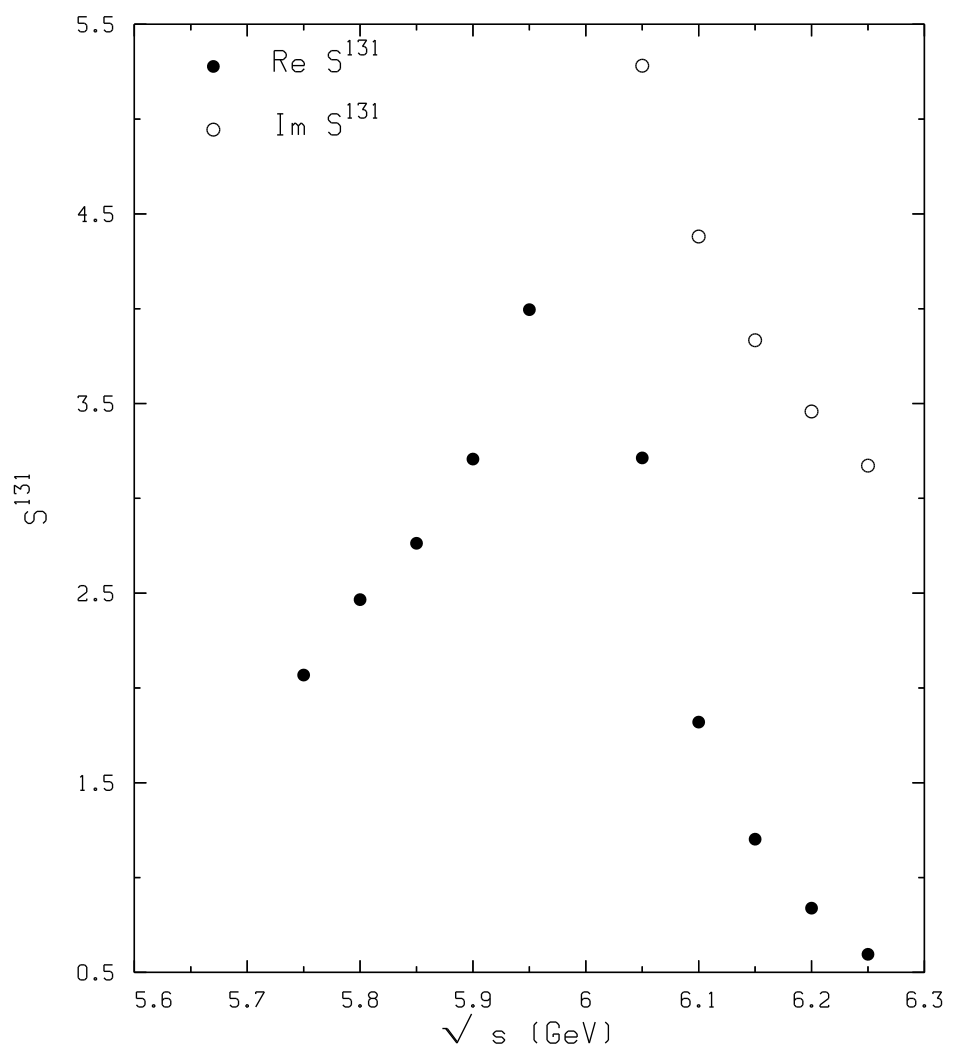

Figure 21: The behavior of $S^{131}$, Fig. 8, around the normal threshold $s=\left(m_{3}+m_{4}\right)^{2}$. 
Already dealing with the sunset topology we discovered this remarkable property that has been generalized now to all two-loop two-point functions but that remains true for all two-loop three-point planar topologies [15]. For all these diagrams the matrix $H$ of Eq.(3) is singular. As a consequence, a change of variables is always possible in the quadratic form $V$ so that the $B$-coefficient of Eq.(1) becomes independent on Feynman parameters, at least if we use only one iteration of the algorithm. In this way we are able to be closer to the original idea of the BTmethod. However, as already stressed in the original paper [6], this $B$ will vanish at some threshold. We have analyzed all Landau singularities for the two-loop self-energies and have to show that this occurs at some non-leading Landau singularity of the diagram. Here some additional analytical work is needed before starting the numerical evaluation.

A second motivation for this work was to start a comprehensive analysis of infrared divergent multi-loop diagrams, from the point of view of their numerical evaluation. Clearly the approach described in this paper is primarily intended for evaluation of massive multi-loop diagrams. However, QED and QCD will be part of any realistic calculation and they usually lead to infrared singularities. Therefore, although the massless world is most efficiently treated in QED and QCD within the analytical approach, any multi-loop calculation in the standard model or beyond, being plagued by infrared divergences, requires to find a way of dealing with them in a purely numerical approach.

For all two-loop two-point functions the relevant infrared objects are the on-shell derivative which are the building block of wave-function renormalization. For some of them the infrared divergent configurations are simple enough to allow for BT treatment with a consequent and straightforward analytical evaluation. The remaining ones, in particular when there is the presence of overlapping infrared configurations, require a novel approach. Our solution is derived by adapting the general algorithm of ref. [17]: the residues of the infrared poles and the finite part of a multi-loop diagram can be cast into a form which allows for a reliable numerical integration.

Work is in progress to extend the numerical evaluation to two-loop vertices, including noninfrared on-shell derivatives of self-energies, where the FORM codes for all topologies, including the non-planar one, have been developed [15].

\section{Acknowledgments}

The contribution of Andrea Ferroglia to the general project of algebraic-numerical evaluation of Feynman diagrams and to several discussions concerning this paper is gratefully acknowledged.

\section{Appendix A}

Consider the topology $S^{111}$ with $m_{1}=m_{2}=0$ and $m_{3}=m$ of Fig. 22. Let us consider the derivative

$$
\pi^{4} \frac{\partial}{\partial p^{2}} S^{111}=-\frac{\mu^{2 \epsilon}}{p^{2}} \int d^{n} q_{1} \int d^{n} q_{2} \frac{p \cdot\left(q_{2}+p\right)}{q_{1}^{2}\left(q_{1}-q_{2}\right)^{2}\left(\left(q_{2}+p\right)^{2}+m^{2}\right)^{2}} .
$$






Figure 22: An $S^{111}$-like contribution to the $W$ self-energy.

We combine the first two propagators with a Feynman parameter $x$, perform the $q_{1}$ integration and combine the two resulting propagators with a new parameter $y$. After the $q_{2}$-integration we put $p^{2}=-m^{2}$ and obtain

$$
\left.S_{p}^{111}\right|_{\mathrm{os}}=\left(\frac{\mu^{2}}{\pi m^{2}}\right)^{\epsilon} \Gamma(\epsilon) B\left(1-\frac{\epsilon}{2}, 1-\frac{\epsilon}{2}\right) B\left(2-2 \epsilon, 1+\frac{\epsilon}{2}\right),
$$

where $B$ is the Euler beta-function. The the on-shell derivative contains a single pole of ultraviolet origin and no infrared pole.

\section{Appendix B}

For completeness we report in this Appendix a list of master integrals 30 that are needed in the evaluation of $S_{p}^{121}$, see Eq.(121). Given $X$ we introduce an auxiliary variable $x$ defined as $X=x-1$ and obtain

$$
\begin{aligned}
& \int_{0}^{1} d y y^{n} \ln y=-\frac{1}{(n+1)^{2}}, \\
& \int_{0}^{1} d y y \ln (1+X y)=\frac{1}{2 X^{2}}\left[\left(X^{2}-1\right) \ln x-\frac{1}{2} X^{2}+X\right], \\
& \int_{0}^{1} d y y \ln \left(1+\frac{y}{X}\right)=\frac{1}{2} X^{2}\left[\left(\frac{1}{X^{2}}-1\right)(\ln x-\ln X)-\frac{1}{2 X^{2}}+\frac{1}{X}\right], \\
& \int_{0}^{1} d y \ln \left(1+\frac{y}{X}\right)=x \ln \left(1+\frac{1}{X}\right)-1, \\
& \int_{0}^{1} d y \ln (1+X y)=\frac{x}{X} \ln x-1, \quad \int_{0}^{1} d y\left(y+\frac{1}{X}\right)^{-1}=\ln x, \\
& \int_{0}^{1} d y y \ln ^{2} y=\frac{1}{4}, \quad \int_{0}^{1} d y \ln ^{2} y=2, \\
& \int_{0}^{1} d y y \ln ^{2}(1+X y)=\frac{1}{X^{2}}\left[x\left(\frac{1}{2} x-1\right) \ln 2 x+x\left(2-\frac{1}{2} x\right) \ln x+\frac{1}{4} x^{2}-2 x+\frac{7}{4}\right], \\
& \int_{0}^{1} d y \ln ^{2}(1+X y)=\frac{1}{X}\left[x \ln ^{2} x-2 x(\ln x-1)-2\right],
\end{aligned}
$$




$$
\begin{aligned}
& \int_{0}^{1} d y\left(y+\frac{1}{X}\right)^{-1} \ln (1+X y)=X \int_{0}^{1} d y(1+X y)^{-1} \ln (1+X y), \\
& \int_{0}^{1} d y(1+X y)^{-1} \ln (1+X y)=\frac{1}{2 X} \ln ^{2} x, \\
& \int_{0}^{1} d y\left(y+\frac{1}{X}\right)^{-1} \ln y=\operatorname{Li}_{2}\left(-\frac{1}{X}\right), \quad \int_{0}^{1} d y y^{-1} \ln \left(1+\frac{y}{X}\right)=-\operatorname{Li}_{2}\left(-\frac{1}{X}\right), \\
& \int_{0}^{1} d y y^{-1} \ln (1+X y)=-\operatorname{Li}_{2}(-X), \\
& \int_{0}^{1} d y y \ln y \ln \left(1+\frac{y}{X}\right)=\frac{1}{4} X^{2}\left(1-\frac{1}{X^{2}}\right)[\ln x-\ln X]-\frac{1}{2} X^{2} \operatorname{Li}_{2}\left(-\frac{1}{X}\right)+\frac{1}{4}-\frac{3}{4} X, \\
& \left.\int_{0}^{1} d y \ln y \ln \left(1+\frac{y}{X}\right)=X\left\{-\left(1+\frac{1}{X}\right)[\ln x-\ln X]+\operatorname{Li}_{2}\left(-\frac{1}{X}\right)\right)\right\}+2, \\
& \int_{0}^{1} d y y \ln y \ln (1+X y)=\frac{1}{4 X^{2}}\left(1-X^{2}\right) \ln x-\frac{1}{2 X^{2}} \operatorname{Li}_{2}(-X)+\frac{1}{4}-\frac{3}{4 X}, \\
& \int_{0}^{1} d y \ln y \ln (1+X y)=\frac{1}{X}\left[-x \ln x+\operatorname{Li} x_{2}(-X)+2,\right. \\
& \int_{0}^{1} d y \ln \left(1+\frac{y}{X}\right)=x[\ln x-\ln X]-1, \\
& \int_{0}^{1} d y(y+X)^{-1}=\ln x-\ln X, \quad \int_{0}^{1} d y y^{n}=\frac{1}{n+1} .
\end{aligned}
$$

\section{Appendix $\mathrm{C}$}

In this appendix we list all the integrals that are relevant to compute the on-shell behavior of $S_{p}^{131}$, see Eq.(227). We will introduce the following terminology:

$$
\mathcal{I}(\mu, \nu, a)=\int_{0}^{1} d x[x(1-x)]^{-\epsilon / 2} \mathcal{J}(\mu, \nu, a), \quad \mathcal{J}(\mu, \nu, a)=\int_{0}^{1} d y y^{\mu}(1+a y)^{\nu} .
$$

We obtain the following results:

$$
\begin{aligned}
\mathcal{J}\left(\frac{\epsilon}{2}-1,-1-\epsilon, X\right) & =\frac{2}{\epsilon}{ }_{2} F_{1}\left(1+\epsilon, \frac{\epsilon}{2} ; 1+\frac{\epsilon}{2} ;-X\right) \\
& =\frac{2}{\epsilon} x^{1+\epsilon}{ }_{2} F_{1}\left(1+\epsilon, 1 ; 1+\frac{\epsilon}{2} ; 1-x\right) .
\end{aligned}
$$

The corresponding $x$ integral gives

$$
\begin{aligned}
\mathcal{I}\left(\frac{\epsilon}{2}-1,-1-\epsilon, X\right) & =\frac{2}{\epsilon} \frac{\Gamma(1+\epsilon / 2)}{\Gamma(1+\epsilon)} \sum_{n=0}^{\infty} \frac{\Gamma(n+1+\epsilon)}{\Gamma(n+1+\epsilon / 2)} \int_{0}^{1} d x x^{1+\epsilon / 2}(1-x)^{n-\epsilon / 2} \\
& =\frac{2}{\epsilon} \frac{\Gamma(1+\epsilon / 2) \Gamma(2+\epsilon / 2)}{\Gamma(1+\epsilon)} \sum_{n=0}^{\infty} \frac{\Gamma(n+1+\epsilon) \Gamma(n+1-\epsilon / 2)}{\Gamma(n+1+\epsilon / 2) \Gamma(n+3)} \\
& =\frac{2}{\epsilon}+1+\mathcal{O}(\epsilon) .
\end{aligned}
$$


A second class of integrals,

$$
\begin{aligned}
& \int_{0}^{1} d y y^{\epsilon / 2}(1+X y)^{-1-\epsilon}=\mathcal{J}\left(\frac{\epsilon}{2},-1-\epsilon, X\right), \\
& \int_{0}^{1} d y y^{-\epsilon / 2}(y+X)^{-1-\epsilon}=X^{-1-\epsilon} \mathcal{J}\left(-\frac{\epsilon}{2},-1-\epsilon, \frac{1}{X}\right),
\end{aligned}
$$

are finite in the limit $\epsilon \rightarrow 0$. We obtain

$$
\int_{0}^{1} d x \int_{0}^{1} d y \frac{1}{1+X y}=\zeta(2)-1, \quad \int_{0}^{1} d x \int_{0}^{1} d y \frac{1}{y+X}=1 .
$$

A third class of integrals can be reduced to the previous ones:

$$
\begin{aligned}
& \int_{0}^{1} d x \int_{0}^{1} d y[x(1-x)]^{-\epsilon / 2} y^{\epsilon / 2-1}(1+X y)^{-\epsilon}= \\
& \int_{0}^{1} d x \int_{0}^{1} d y[x(1-x)]^{-\epsilon / 2}\left\{\frac{2}{\epsilon}(1+X)^{-\epsilon}+2 \ln (1+X)+\epsilon\left[\operatorname{Li}_{2}(-X)-\ln ^{2}(1+X)\right]\right\} \\
= & \int_{0}^{1} d x\left\{\frac{2}{\epsilon} x^{\epsilon / 2}(1-x)^{-\epsilon / 2}-2 \ln x+\epsilon\left[\operatorname{Li}_{2}\left(\frac{x-1}{x}\right)+\ln (1-x) \ln x\right]\right\} \\
= & \frac{2}{\epsilon} \Gamma\left(1+\frac{\epsilon}{2}\right) \Gamma\left(1-\frac{\epsilon}{2}\right)+2+\epsilon \mathcal{C}=\frac{2}{\epsilon}+2+\left[\mathcal{C}+\frac{1}{2} \psi^{\prime}(1)\right] \epsilon,
\end{aligned}
$$

where we used the relation

$$
\Gamma\left(1 \pm \frac{\epsilon}{2}\right)=1 \mp \frac{1}{2} \gamma \epsilon+\frac{1}{8}\left[\gamma^{2}+\psi^{\prime}(1)\right] \epsilon^{2}+\mathcal{O}\left(\epsilon^{3}\right) .
$$

The constant is

$$
\mathcal{C}=\int_{0}^{1} d x\left[\operatorname{Li}_{2}(x)-\frac{1}{2} \ln ^{2} x+2 \ln x \ln (1-x)-\zeta(2)\right]
$$

where we used

$$
\mathrm{Li}_{2}\left(\frac{x-1}{x}\right)=\mathrm{Li}_{2}(x)+\ln x \ln (1-x)-\frac{1}{2} \ln ^{2} x-\zeta(2) .
$$

After integration we obtain

$$
\mathcal{C}=2-2 \zeta(2)
$$

where

$$
\int_{0}^{1} d x\left\{\operatorname{Li}_{2}(x) ; \ln x \ln (1-x) ; \ln ^{2} x\right\}=\{\zeta(2)-1 ; 2-\zeta(2) ; 2\} .
$$

Similarly

$$
\int_{0}^{1} d x \int_{0}^{1} d y[x(1-x)]^{-\epsilon / 2} y^{-\epsilon / 2-1}(y+X)^{-\epsilon}=-\frac{2}{\epsilon}-2-\left[2+\frac{5}{2} \zeta(2)\right] \epsilon+\mathcal{O}\left(\epsilon^{2}\right) .
$$




\section{Appendix D}

In this Appendix we report the explicit expression for the residue of the single infrared pole $\mathcal{S}_{221}^{1}$ defined in Eq. (305).

$$
\begin{aligned}
\mathcal{S}_{221}^{1} & =\int_{0}^{1} d x_{2} \int_{0}^{1} d x_{1} \int_{0}^{1} d y\left\{x_{2}\left(1+x_{1}\right)\left[\left(1+x_{1}\right)^{2}-\left(1-x_{1} x_{2}\right)^{2} x_{2}^{2} y^{2}\right] A_{011}^{-2}\right. \\
& +x_{2}^{2} y\left(1+x_{1}\right)\left[\left(1+x_{1}\right)^{2}-\left(1-x_{1} x_{2} y\right)^{2} y^{2}\right] A_{012}^{-2} \\
& +x_{1}\left(1+x_{2}\right)\left[\left(1+x_{2}\right)^{2}-\left(1-x_{1}\right)^{2} x_{1}^{2} x_{2}^{2} y^{2}\right] A_{021}^{-2} \\
& +x_{1}^{2} y\left(1+x_{2}\right)\left[\left(1+x_{2}\right)^{2}-\left(1-x_{1} y\right)^{2} x_{2}^{2} y^{2}\right] A_{022}^{-2} \\
& +\left(1+x_{2}-x_{1} x_{2}^{2}\right)\left[\left(1+x_{2}-x_{1} x_{2}^{2}\right)^{2}-x_{1}^{2} x_{2}^{2} y^{2}\right] A_{1}^{-2} \\
& +\left(1+x_{1} x_{2}-x_{1}^{2} x_{2}\right)\left[\left(1+x_{1} x_{2}-x_{1}^{2} x_{2}\right)^{2}-x_{1}^{2} x_{2}^{2} y^{2}\right] A_{21}^{-2} \\
& \left.+x_{2}\left(1+x_{1} x_{2} y-x_{1}^{2} x_{2} y\right)\left[\left(1+x_{1} x_{2} y-x_{1}^{2} x_{2} y\right)^{2}-x_{1}^{2} y^{2}\right] A_{22}^{-2}+1\right\} \\
& +\int_{0}^{\infty} d x_{2} \int_{0}^{1} d x_{1} \int_{0}^{1} d y \frac{x_{2}}{1+x_{2}}\left\{\left[\frac{\left(1-x_{1}\right)^{3} B_{1}^{-2}}{x_{1}}\right]_{+}+\frac{\left(1-x_{1}\right)^{2}}{1+x_{2}} B_{1}^{-2}\right\} \\
& +\frac{1}{2} \int_{0}^{\infty} d x_{2} \int_{0}^{1} d y \frac{1}{1+x_{2}} B_{1}^{-2}\left(x_{1}=0\right)\left[x_{2} L_{b 1}\left(x_{1}=0\right)+1\right] \\
& +\int_{1}^{\infty} d x_{2} \int_{0}^{1} d x_{1} \int_{0}^{1} d y \frac{x_{2}}{1+x_{2}}\left\{\left[\frac{\left(1-x_{1} y\right)^{3} B_{2}^{-2}}{y}\right]_{+}+\frac{\left(1-x_{1} y\right)^{2}}{1+x_{2}} B_{2}^{-2}\right\} \\
& +\frac{1}{2} \int_{1}^{\infty} d x_{2} \int_{0}^{1} d x_{1} \frac{1}{1+x_{2}} B_{2}^{-2}(y=0)\left[x_{2} L_{b 2}(y=0)+1\right] \\
& +\int_{0}^{1} d x_{2} \int_{0}^{1} d x_{1} \int_{0}^{1} d y\left\{\frac{1}{1+x_{2}}\left[\frac{\left(1-x_{1} x_{2} y\right)^{3} B_{21}^{-2}}{y}\right]_{+}\right. \\
& +\frac{x_{1} x_{2}}{\left(1+x_{2}\right)^{2}}\left(1-x_{1} x_{2} y\right)^{2} B_{21}^{-2}+\frac{x_{2}}{1+x_{1} x_{2}}\left[\frac{\left(1-x_{1} y\right)^{3} B_{22}^{-2}}{y}\right]_{+} \\
& \left.+\frac{x_{1} x_{2}}{\left(1+x_{1} x_{2}\right)^{2}}\left(1-x_{1} y\right)^{2} B_{22}^{-2}\right\} \\
& +\frac{1}{2} \int_{0}^{1} d x_{2} \int_{0}^{1} d x_{1}\left\{\left[\frac{B_{21}^{-2}(y=0)}{\left(1+x_{2}\right) x_{2}}\right]_{+}\right. \\
& +\frac{1}{1+x_{2}} B_{21}^{-2}(y=0)\left[L_{b 21}(y=0)+\ln x_{2}\right]+\left[\frac{B_{22}^{-2}(y=0)}{\left.\left(1+x_{1} x_{2}\right) x_{2}\right]_{+}}\right. \\
& \left.+\frac{x_{2}}{1+x_{1} x_{2}} B_{22}^{-2}(y=0)\left[L_{b 22}(y=0)+\ln x_{1}\right]\right\} \\
& +\frac{1}{2} \int_{0}^{1} d x_{1} B_{21}^{-2}\left(y=x_{2}=0\right)\left[L_{b 21}\left(y=x_{2}=0\right)-1\right] \\
& \frac{1}{2} x_{2}^{-2}\left(y=x_{1}=0\right)\left[L_{b 22}\left(y=x_{1}=0\right)-1\right] \\
&
\end{aligned}
$$


where the following functions have been introduced:

$$
\begin{aligned}
A_{011} & =\left(1+x_{1}\right)^{2}+\left(1+x_{2}^{2}\right)\left(1+x_{1}\right)\left(1-x_{1} x_{2}\right) y+\left(1-x_{1} x_{2}\right)^{2} x_{2}^{2} y^{2} \\
A_{012} & =\left(1+x_{1}\right)^{2} x_{2}+\left(1+x_{2}^{2} y^{2}\right)\left(1+x_{1}\right)\left(1-x_{1} x_{2} y\right)+\left(1-x_{1} x_{2} y\right)^{2} x_{2} y^{2} \\
A_{021} & =\left(1+x_{2}\right)^{2}+\left(1+x_{1}^{2} x_{2}^{2}\right)\left(1+x_{2}\right)\left(1-x_{1}\right) y+\left(1-x_{1}\right)^{2} x_{1}^{2} x_{2}^{2} y^{2} \\
A_{022} & =\left(1+x_{2}\right)^{2} x_{1}+\left(1+x_{1}^{2} x_{2}^{2} y^{2}\right)\left(1+x_{2}\right)\left(1-x_{1} y\right)+\left(1-x_{1} y\right)^{2} x_{1} x_{2}^{2} y^{2} \\
A_{1} & =\left(1+x_{2}-x_{1} x_{2}^{2}\right)^{2}+\left(1+x_{2}^{2}\right)\left(1+x_{2}-x_{1} x_{2}^{2}\right) x_{1} y+x_{1}^{2} x_{2}^{2} y^{2} \\
A_{21} & =\left(1+x_{1} x_{2}-x_{1}^{2} x_{2}\right)^{2}+\left(1+x_{1}^{2} x_{2}^{2}\right)\left(1+x_{1} x_{2}-x_{1}^{2} x_{2}\right) y+x_{1}^{2} x_{2}^{2} y^{2} \\
A_{22} & =\left(1+x_{1} x_{2} y-x_{1}^{2} x_{2} y\right)^{2} x_{2}^{2}+\left(1+x_{1}^{2} x_{2}^{2} y^{2}\right)\left(1+x_{1} x_{2} y-x_{1}^{2} x_{2} y\right)+x_{1}^{2} x_{2} y^{2} \\
B_{1} & =1+x_{2}-x_{1} x_{2}+\left(1-x_{1}\right)^{2} x_{2} y \\
B_{2} & =\left(1+x_{2}-x_{1} x_{2} y\right) x_{1}+\left(1-x_{1} y\right)^{2} x_{2} \\
B_{21} & =\left(1+x_{2}-x_{1} x_{2}^{2} y\right) x_{1}+\left(1-x_{1} x_{2} y\right)^{2} \\
B_{22} & =\left(1+x_{1} x_{2}-x_{1}^{2} x_{2} y\right)+\left(1-x_{1} y\right)^{2} x_{2}
\end{aligned}
$$

and also

$$
\begin{aligned}
L_{a 011} & =\ln \left(A_{011}\right)-\frac{1}{2} \ln y-\ln \left(1+x_{2}\right)+\frac{1}{2} \ln \left(1-x_{1} x_{2}\right)-\frac{3}{2} \ln \left(1+x_{1}\right) \\
L_{a 012} & =\ln \left(A_{012}\right)-\frac{1}{2} \ln x_{2}-\ln \left(1+x_{2} y\right)+\frac{1}{2} \ln \left(1-x_{1} x_{2} y\right)-\frac{3}{2} \ln \left(1+x_{1}\right) \\
L_{a 021} & =\ln \left(A_{021}\right)-\frac{1}{2} \ln y-\ln \left(1+x_{1} x_{2}\right)+\frac{1}{2} \ln \left(1-x_{1}\right)-\frac{3}{2} \ln \left(1+x_{2}\right) \\
L_{a 022} & =\ln \left(A_{022}\right)-\frac{1}{2} \ln x_{1}-\ln \left(1+x_{1} x_{2} y\right)+\frac{1}{2} \ln \left(1-x_{1} y\right)-\frac{3}{2} \ln \left(1+x_{2}\right) \\
L_{a 1} & =\ln \left(A_{1}\right)-\frac{1}{2} \ln y+\frac{1}{2} \ln x_{1}+\ln x_{2}-\ln \left(1+x_{2}\right)-\frac{3}{2} \ln \left(1+x_{2}-x_{1} x_{2}^{2}\right) \\
L_{a 21} & =\ln \left(A_{21}\right)-\frac{1}{2} \ln y+\ln x_{1}-\ln \left(1+x_{1} x_{2}\right)-\frac{3}{2} \ln \left(1+x_{1} x_{2}-x_{1}^{2} x_{2}\right) \\
L_{a 22} & =\ln \left(A_{22}\right)+\ln x_{1}-\frac{1}{2} \ln x_{2}-\ln \left(1+x_{1} x_{2} y\right)-\frac{3}{2} \ln \left(1+x_{1} x_{2} y-x_{1}^{2} x_{2} y\right) \\
L_{b 1} & =\ln \left(B_{1}\right)+\frac{1}{2} \ln y-\frac{1}{2} \ln x_{2}+\ln \left(1+x_{2}\right)-\frac{3}{2} \ln \left(1+x_{2}-x_{1} x_{2}\right) \\
L_{b 2} & =\ln \left(B_{2}\right)+\frac{1}{2} \ln x_{1}-\frac{1}{2} \ln x_{2}+\ln \left(1+x_{2}\right)-\frac{3}{2} \ln \left(1+x_{2}-x_{1} x_{2} y\right) \\
L_{b 21} & =\ln \left(B_{21}\right)+\frac{1}{2} \ln x_{1}+\ln \left(1+x_{2}\right)-\frac{3}{2} \ln \left(1+x_{2}-x_{1} x_{2}^{2} y\right) \\
L_{b 22} & =\ln \left(B_{22}\right)-\frac{1}{2} \ln x_{2}+\ln \left(1+x_{1} x_{2}\right)-\frac{3}{2} \ln \left(1+x_{1} x_{2}-x_{1}^{2} x_{2} y\right) .
\end{aligned}
$$




\section{Appendix E}

In this Appendix we consider all those results that are useful to derive analytical expressions for the imaginary parts of two-loop two-point functions. The two-particle cut contribution to the discontinuity of $S^{121}$, shown in Fig. 5 , is given by

$$
\operatorname{disc}_{2} S^{121}=-2 i B_{0}\left(-m_{3}^{2} ; m_{1}, m_{2}\right) \operatorname{Im} B_{0}\left(-s ; m_{3}, m_{4}\right) .
$$

Since the one-loop scalar function $B_{0}$ is

$$
B_{0}\left(p^{2} ; m_{1}, m_{2}\right)=\frac{1}{\bar{\varepsilon}}-\ln \frac{m_{1} m_{2}}{\mu^{2}}-R+\frac{m_{1}^{2}-m_{2}^{2}}{2 p^{2}} \ln \frac{m_{1}^{2}}{m_{2}^{2}}+2,
$$

where

$$
R=-\frac{\Lambda}{p^{2}} \ln \frac{p^{2}-i \delta+m_{1}^{2}+m_{2}^{2}-\Lambda}{2 m_{1} m_{2}},
$$

and where $\Lambda^{2}=\lambda\left(-p^{2}, m_{1}^{2}, m_{2}^{2}\right)$, we must have the imaginary part of $B_{0}\left(-s ; m_{3}, m_{4}\right)$ to $\mathcal{O}(\epsilon)$. It follows

$$
\begin{aligned}
B_{0}\left(p^{2} ; m_{1}, m_{2}\right) & =\frac{1}{\bar{\varepsilon}}-\int_{0}^{1} d x \ln \frac{\chi}{\mu^{2}} \\
& +\epsilon\left\{\frac{1}{4}(\gamma+\ln \pi)^{2}+\frac{1}{4} \zeta(2)+\frac{1}{2} \int_{0}^{1} d x \ln \frac{\chi}{\mu^{2}}\left[\gamma+\ln \pi+\frac{1}{2} \ln \frac{\chi}{\mu^{2}}\right]\right\}
\end{aligned}
$$

where, as usual, $\chi=-p^{2} x^{2}+\left(p^{2}+m_{2}^{2}-m_{1}^{2}\right) x+m_{1}^{2}$. Note that, for $s>\left(m_{3}+m_{4}\right)^{2}$ and $m_{3}^{2}>$ $\left(m_{1}+m_{2}\right)^{2}$ the discontinuity develops a real part. However, in the same region the discontinuity coming from the three-particle cut of Fig. 23 also has a real part and the two cancel so that $\operatorname{disc}_{2+3} S_{121}$ is purely imaginary. For completeness we report the result for $\operatorname{disc}_{3} S^{121}$. Define first

$$
\begin{aligned}
s_{ \pm \pm} & =\left(\sqrt{s} \pm m_{4}\right)^{2}-\left(m_{2} \pm m_{1}\right)^{2}, \quad \kappa^{2}=\frac{s_{ \pm} s_{\mp}}{s_{++} s_{--}} \\
x_{3} & =\frac{q_{\mp}}{q_{ \pm}}, \quad c_{1}=\sqrt{s_{++} s_{--}}
\end{aligned}
$$

and also

$$
\begin{aligned}
& \frac{c_{2}}{c_{1}}=4 m_{1} m_{2} s_{--}, \quad \frac{c_{3}}{c_{1}}=8 m_{1} m_{2}\left(s-m_{3}^{2}+m_{1}^{2}+m_{2}^{2}+m_{4}^{2}\right), \\
& \frac{c_{4}}{c_{1}}=-8 m_{1} m_{2} \frac{\left(s-m_{4}^{2}\right)^{2}}{m_{3}^{2}}, \quad \frac{c_{5}}{c_{1}}=8 m_{1} m_{2} \frac{\lambda\left(s, m_{3}^{2}, m_{4}^{2}\right)}{m_{3}^{2}} \\
& \frac{x_{4}}{x_{3}}=\frac{\left(m_{2}-m_{1}\right)^{2}}{\left(m_{2}+m_{1}\right)^{2}}, \quad \frac{x_{5}}{x_{3}}=\frac{m_{3}^{2}-\left(m_{2}-m_{1}\right)^{2}}{m_{3}^{2}-\left(m_{2}+m_{1}\right)^{2}}
\end{aligned}
$$

It follows

$$
\begin{aligned}
\operatorname{disc}_{3} S^{121} & =\frac{2 i \pi}{s}\left[c_{1} E(\kappa)+c_{2} K(\kappa)+c_{3} \Pi\left(x_{3}, \kappa\right)\right. \\
& \left.+c_{4} \Pi\left(x_{4}, \kappa\right)+c_{5} \Pi\left(x_{5}-i \delta, \kappa\right)\right] \theta\left(s-\left(m_{1}+m_{2}+m_{4}\right)^{2}\right)
\end{aligned}
$$


where $E, K$ and $\Pi$ are elliptic integrals [24]. The $i \delta$ prescription is needed because in the last complete elliptic integral of third kind we have, in the region $s>\left(m_{3}+m_{4}\right)^{2}$ and $m_{3}^{2}>\left(m_{1}+m_{2}\right)^{2}$, that $x_{5}>1$ and the integral must be understood as a Cauchy principal value. The corresponding imaginary part cancel the real part from disc $c_{2}$. Adding the two contributions we find

$$
\operatorname{Im} S^{121}=-\frac{i}{2}\left[\operatorname{disc}_{2} S^{121}+\operatorname{disc}_{3} S^{121}\right] .
$$

For a numerical evaluation of elliptic integrals we use the NAG sub-routines S21BAF - S21BDF,



Figure 23: The three-particle cut of diagram $S^{121}$

following the alternative definition by Carlson [25].

\section{Appendix F}

In this Appendix we report some special cases of $S^{221}$ for which the low-(high-) $p^{2}$ expansion is known [26]. Let us define the following variables

$$
x=-\frac{m^{2}}{s}+i \delta, \quad y=-\frac{s}{m^{2}}-i \delta .
$$

We obtain the following two expansions:

$$
\begin{aligned}
& S^{221}(s ; m, 0,0,0,0)=\frac{1}{s} \sum_{n=1}^{\infty}\left[\frac{\ln ^{2} y+2 \zeta(2)}{2 n}-\frac{2}{n^{2}} \ln y+\frac{3}{n^{3}}+r_{2}(n)\right](-y)^{n}, \quad|y| \ll 1, \\
& S^{221}(s ; m, 0,0,0,0)=-\frac{1}{s} \sum_{n=1}^{\infty}\left[\frac{\ln ^{2} x+2 \zeta(2)}{2 n}-\frac{2}{n^{2}} \ln x+\frac{3}{n^{3}}+r_{2}(n)\right](-x)^{n}-6 \frac{\zeta(3)}{s}, \quad|x| \ll 1,
\end{aligned}
$$

where $r_{2}$ is defined as

$$
r_{2}(n)=\frac{1}{n^{2}} \sum_{l=1}^{n-1} \frac{n-l}{l^{2}} .
$$

Another known expansion is the following:

$$
S^{221}(s ; m, m, m, 0,0)=\frac{1}{2 m^{2}}, \sum_{n=0}^{\infty} \frac{n ! \Gamma(3 / 2)}{\Gamma(n+3 / 2)}\left(\frac{s}{4 m^{2}}\right)^{n}\left[\frac{3}{(n+1)^{2}}-\frac{\ln y}{n+1}\right] .
$$




\begin{tabular}{|l|l|l|l|l|}
\hline$\sqrt{s}[\mathrm{GeV}]$ & $\operatorname{Re} S^{121}$ & $\operatorname{Re} S^{121}$ (anal.) & $\operatorname{Im} S^{121}$ & $\operatorname{Im} S^{121}$ (anal.) \\
\hline 75 & $\begin{array}{l}54.27(3) \\
54.2652(1)\end{array}$ & 54.2624 & 0.00 & 0.00 \\
\hline 77 & $\begin{array}{l}53.11(3) \\
53.1227(4)\end{array}$ & 53.1076 & 0.00 & 0.00 \\
\hline 79 & $\begin{array}{l}51.63(3) \\
51.6348(2)\end{array}$ & 51.6333 & 0.00 & 0.00 \\
\hline 80 & $\begin{array}{l}50.59(3) \\
50.6040(2)\end{array}$ & 50.5868 & 0.00 & 0.00 \\
\hline 81 & $\begin{array}{l}49.35(3) \\
49.3791(4)\end{array}$ & 49.3415 & $\begin{array}{l}-0.58(1) \\
-0.5850(5)\end{array}$ & -0.585457 \\
\hline 85 & $\begin{array}{l}47.68(3) \\
47.6781(4)\end{array}$ & $47.63(3)$ & 46.5277 & $-3.88(2)$ \\
\hline \hline
\end{tabular}

Table 1: The topology $S^{11}$ for $m_{1}=m_{3}=80 \mathrm{GeV}$ and $m_{2}=m_{4}=0$ as a function of $\sqrt{s}$. First entry is from Eq.(79) and NAG subroutine D01EAF and the analytical result of ref. [26]. Second entry is from Eq.(79) and NAG subroutine D01BGF. The UV-pole is $\epsilon=1$ and the unit of mass is $\mu=1 \mathrm{GeV}$. 


\begin{tabular}{|c|c|c|c|c|}
\hline$\sqrt{s}[\mathrm{GeV}]$ & $\operatorname{Re} S^{121}$ & $\operatorname{Re} S^{121}$ (anal.) & $\operatorname{Im} S^{121}$ & $\operatorname{Im} S^{121}$ (anal.) \\
\hline 75 & $\begin{array}{l}40.34(3) \\
40.3501(4)\end{array}$ & 40.3502 & $\begin{array}{l}-49.77(3) \\
-49.7756(3)\end{array}$ & -49.7720 \\
\hline 77 & $\begin{array}{l}41.16(3) \\
41.1952(5)\end{array}$ & 41.1696 & $\begin{array}{l}-49.94(3) \\
-49.9387(5)\end{array}$ & -49.9374 \\
\hline 79 & $\begin{array}{l}41.96(3) \\
41.9512(4)\end{array}$ & 41.9693 & $\begin{array}{l}-50.10(3) \\
-50.1045(3)\end{array}$ & -50.0985 \\
\hline 80 & $\begin{array}{l}42.36(3) \\
42.3447(4)\end{array}$ & 42.3621 & $\begin{array}{l}-50.18(3) \\
-50.1756(3)\end{array}$ & -50.1775 \\
\hline 81 & $\begin{array}{l}42.74(3) \\
42.7302(4)\end{array}$ & 42.7502 & $\begin{array}{l}-50.26(3) \\
-50.2691(3)\end{array}$ & -50.2556 \\
\hline 83 & $\begin{array}{l}43.50(3) \\
43.5399(4)\end{array}$ & 43.5127 & $\begin{array}{l}-50.41(3) \\
-50.4033(4)\end{array}$ & -50.4090 \\
\hline 85 & $\begin{array}{l}44.25(4) \\
44.2784(4)\end{array}$ & 44.2576 & $\begin{array}{l}-50.57(3) \\
-50.5566(2)\end{array}$ & -50.5593 \\
\hline
\end{tabular}

Table 2: The topology $S^{11}$ for $m_{1}=80 \mathrm{GeV}$ and $m_{2}=m_{3}=m_{4}=0$ as a function of $\sqrt{s}$. First entry is from Eq.(79) and NAG subroutine D01EAF and the analytical result of ref. [26]. Second entry is from Eq.(79) and NAG subroutine D01BGF. The UV-pole is $\epsilon=1$ and the unit of mass is $\mu=1 \mathrm{GeV}$. 


\begin{tabular}{|l|l|l|l|}
\hline$\sqrt{s}[\mathrm{GeV}]$ & $\operatorname{Im} S^{121}$ & $\operatorname{Im} S^{121}$ & $\operatorname{Im} S^{121}$ (anal.) \\
\hline above two-p cut & & & \\
\hline \hline 180 & $15.90(3)$ & $15.8909(5)$ & 15.8957 \\
\hline 190 & $23.26(3)$ & $23.2562(5)$ & 23.2592 \\
\hline 200 & $28.41(4)$ & $28.4148(4)$ & 28.4059 \\
\hline 210 & $32.39(4)$ & $32.3947(5)$ & 32.3834 \\
\hline 220 & $35.62(4)$ & $35.6124(4)$ & 35.6137 \\
\hline 230 & $38.30(4)$ & $38.2994(4)$ & 38.2953 \\
\hline 240 & $40.59(4)$ & $40.5769(5)$ & 40.5929 \\
\hline 250 & $42.57(4)$ & $42.5749(5)$ & 42.5613 \\
\hline \hline
\end{tabular}

Table 3: The imaginary part of $S^{121}$ for $m_{1}=m_{3}=80 \mathrm{GeV}$ and $m_{2}=m_{4}=91 \mathrm{GeV}$ as a function of $\sqrt{s}$. First entry is from Eq. (79) and NAG subroutine D01EAF. Second entry is from the same equation and NAG subroutine D01BGF and third one is the analytical result of ref. [26] as reported in Appendix E. 


\begin{tabular}{|l|l|l|l|}
\hline$\sqrt{s}[\mathrm{GeV}]$ & $\operatorname{Im} S^{121}$ & $\operatorname{Im} S^{121}$ & $\operatorname{Im} S^{121}$ (anal.) \\
\hline above three-p cut & & & \\
\hline \hline 260 & $44.30(5)$ & $44.3069(5)$ & 44.2915 \\
\hline 270 & $45.84(4)$ & $45.8391(5)$ & 45.8339 \\
\hline 280 & $47.24(4)$ & $47.2457(5)$ & 47.2316 \\
\hline \hline
\end{tabular}

Table 4: The imaginary part of $S^{121}$ for $m_{1}=m_{3}=80 \mathrm{GeV}$ and $m_{2}=m_{4}=91 \mathrm{GeV}$ as a function of $\sqrt{s}$. First entry is from Eq. (79) and NAG subroutine D01EAF. Second entry is from the same equation and NAG subroutine D01BGF and third one is the analytical result of ref. [26] as reported in Appendix E. 


\begin{tabular}{|c|c|c|c|c|c|}
\hline$\sqrt{s}[\mathrm{GeV}]$ & $\operatorname{Im} S^{121}(\mathrm{I})$ & $\operatorname{Im} S^{121}(I I)$ & $\operatorname{Im} S^{121}$ (anal.) & $\mid \mathrm{I} /$ anal - $1 \mid$ & $\mid$ II/anal - 1| \\
\hline \multicolumn{6}{|c|}{ around the two-p cut } \\
\hline 171.1 & $1.26(1)$ & $1.25423(5)$ & 1.24533 & $0.87 \%$ & $0.71 \%$ \\
\hline 171.2 & $1.89(1)$ & $1.87835(5)$ & 1.87846 & $0.13 \%$ & $0.01 \%$ \\
\hline 171.3 & $2.38(1)$ & $2.37516(5)$ & 2.37086 & $0.28 \%$ & $0.18 \%$ \\
\hline 171.4 & $2.80(2)$ & $2.80222(5)$ & 2.79280 & $0.43 \%$ & $0.34 \%$ \\
\hline \multicolumn{6}{|c|}{ around the three-p cut } \\
\hline 262.1 & $44.63(4)$ & $44.6222(5)$ & 44.6337 & $<0.01 \%$ & $0.03 \%$ \\
\hline 262.2 & $44.65(4)$ & $44.6446(3)$ & 44.6392 & $0.01 \%$ & $0.03 \%$ \\
\hline 262.3 & $44.67(4)$ & $44.6617(4)$ & 44.6589 & $0.02 \%$ & $0.01 \%$ \\
\hline 262.4 & $44.68(4)$ & $44.6983(5)$ & 44.6718 & $0.02 \%$ & $0.06 \%$ \\
\hline
\end{tabular}

Table 5: A scan of the imaginary part of $S^{121}$ for $m_{1}=m_{3}=80 \mathrm{GeV}$ and $m_{2}=m_{4}=91 \mathrm{GeV}$ around the two- and three-particle cuts. First entry is from Eq.(79) and NAG subroutine D01EAF. Second entry is from Eq. (79) and NAG subroutine D01BGF and third one is the analytical result of ref. [26] as reported in Appendix E. 


\begin{tabular}{|l|l|l|}
\hline$\sqrt{s}[\mathrm{GeV}]$ & $\operatorname{Im} S^{131}$ & $-\frac{\partial}{\partial m_{3}^{2}} \operatorname{Im} S^{121}$ \\
\hline \hline 7 & $-1.551(2)$ & $-1.551(3)$ \\
\hline 12 & $-0.3047(3)$ & $-0.3047(4)$ \\
\hline 17 & $-0.1494(1)$ & $-0.1494(11)$ \\
\hline 22 & $-0.09166(9)$ & $-0.09183(282)$ \\
\hline \hline
\end{tabular}

Table 6: A comparison between $S^{131}$ and the numerical derivative of $S^{121}$ for $m_{3}=m_{5}=1 \mathrm{GeV}$ and $m_{1}=m_{2}=m_{4}=5 \mathrm{GeV}$ as a function of $\sqrt{s}$. The UV-pole is $\epsilon=1$ and the unit of mass is $\mu=1 \mathrm{GeV}$. 


\begin{tabular}{|l|l|l|l|}
\hline$\sqrt{s}[\mathrm{GeV}]$ & $\operatorname{Re} S^{131}$ & $\operatorname{Im} S^{131}$ & $-\frac{\partial}{\partial m_{3}^{2}} \operatorname{Im} S^{121}$ \\
\hline \hline 5.80 & $-2.46581(1)$ & 0.00 & 0.00 \\
\hline 5.85 & $-2.76299(1)$ & 0.00 & 0.00 \\
\hline 5.90 & $-3.20714(1)$ & 0.00 & 0.00 \\
\hline 5.95 & $-3.99512(2)$ & 0.00 & 0.00 \\
\hline 6.05 & $-3.21(1)$ & $-5.28(1)$ & $-5.2794(5)$ \\
\hline 6.10 & $-1.820(2)$ & $-4.381(4)$ & $-4.3735(8)$ \\
\hline 6.15 & $-1.202(2)$ & $-3.834(1)$ & $-3.8354(3)$ \\
\hline 6.20 & $-0.8387(5)$ & $-3.458(1)$ & $-3.4588(5)$ \\
\hline 6.25 & $-0.5954(5)$ & $-3.1725(7)$ & $-3.1719(4)$ \\
\hline \hline
\end{tabular}

Table 7: $S^{131}$ and the numerical derivative of $S^{121}$ for $m_{3}=m_{5}=1 \mathrm{GeV}$ and $m_{1}=m_{2}=m_{4}=5 \mathrm{GeV}$ around the normal threshold $\sqrt{s}=6 \mathrm{GeV}$. The UV-pole is $\epsilon=1$ and the unit of mass is $\mu=1 \mathrm{GeV}$. 


\begin{tabular}{|c|c|c|c|c|}
\hline$s\left[\mathrm{GeV}^{2}\right]$ & $\operatorname{Re} S^{221}$ & Re $S^{221}$ ref. 27 & $\operatorname{Im} S^{221}$ & $\operatorname{Im} S^{221}$ ref. 27 \\
\hline 0.1 & $\begin{array}{l}-0.287205 \pm 0.20 \times 10^{-4} \\
-0.287237 \pm 0.35 \times 10^{-5}\end{array}$ & $-0.287238 \pm 0.25 \times 10^{-5}$ & 0.00 & 0.00 \\
\hline 0.5 & $\begin{array}{l}-0.294578 \pm 0.15 \times 10^{-4} \\
-0.294590 \pm 0.23 \times 10^{-5}\end{array}$ & $-0.294952 \pm 0.25 \times 10^{-5}$ & 0.00 & 0.00 \\
\hline 1.0 & $\begin{array}{l}-0.304543 \pm 0.21 \times 10^{-4} \\
-0.304522 \pm 0.22 \times 10^{-5}\end{array}$ & $-0.304521 \pm 0.25 \times 10^{-5}$ & 0.00 & 0.00 \\
\hline 5.0 & $\begin{array}{l}-0.452499 \pm 0.17 \times 10^{-4} \\
-0.452518 \pm 0.31 \times 10^{-4}\end{array}$ & $-0.452520 \pm 0.28 \times 10^{-5}$ & 0.00 & 0.00 \\
\hline 10.0 & $\begin{array}{l}-0.488036 \pm 0.59 \times 10^{-3} \\
-0.488224 \pm 0.18 \times 10^{-3}\end{array}$ & $-0.488153 \pm 0.23 \times 10^{-5}$ & $\begin{array}{l}-0.353197 \pm 0.69 \times 10^{-3} \\
-0.353082 \pm 0.18 \times 10^{-3}\end{array}$ & $-0.353217 \pm 0.23 \times 10^{-5}$ \\
\hline 50.0 & $\begin{array}{l}0.173897 \pm 0.49 \times 10^{-4} \\
0.173900 \pm 0.18 \times 10^{-4}\end{array}$ & $0.173901 \pm 0.22 \times 10^{-5}$ & $\begin{array}{l}-0.118089 \pm 0.40 \times 10^{-4} \\
-0.118082 \pm 0.20 \times 10^{-4}\end{array}$ & $-0.118080 \pm 0.22 \times 10^{-5}$ \\
\hline
\end{tabular}

Table 8: $S^{221}$ compared with the numerical result of Tab. 1 in ref. [27]. Here $m_{n}^{2}=n \mathrm{GeV}^{2}$, for $n=1, \ldots, 5$. The second entry refers to a run with a much larger number of points in sub-routine D01GDF. 


\begin{tabular}{|l|l|l|l|l|}
\hline$s\left[\mathrm{GeV}^{2}\right]$ & $\operatorname{Re} S^{221}$ & $\operatorname{Re} S^{221}$ expansion & $\operatorname{Im} S^{221}$ & $\operatorname{Im} S^{221}$ expansion \\
\hline \hline 0.1 & $0.3740(6)$ & 0.373988 & $0.27957(9)$ & 0.279970 \\
\hline 1.0 & $0.19599(4)$ & 0.195930 & $0.20847(6)$ & 0.208394 \\
\hline 10.0 & $0.07144(3)$ & 0.071467 & $0.14067(3)$ & 0.140692 \\
\hline \hline 5.0 & $-1.61744(9)$ & -1.61746 & $0.4909(2)$ & 0.490807 \\
\hline 10.0 & $-0.79270(2)$ & -0.79270 & $0.14061(5)$ & 0.140692 \\
\hline 50.0 & $-0.150366(2)$ & -0.150362 & $0.007492(3)$ & 0.007492 \\
\hline \hline
\end{tabular}

Table 9: $S^{221}$ compared with the expansions described in Appendix F for $m_{1}=100 \mathrm{GeV}\left(m_{1}=1 \mathrm{GeV}\right)$ upper(lower) part of the table. The sum over $n$ in Eq.422) is restricted to $n \leq 20$. 


\begin{tabular}{|l|l|l|l|l|}
\hline$s\left[\mathrm{GeV}^{2}\right]$ & $\operatorname{Re} S^{221}$ & $\operatorname{Re} S^{221}$ expansion & $\operatorname{Im} S^{221}$ & $\operatorname{Im} S^{221}$ expansion \\
\hline \hline 0.1 & $0.0496(2)$ & 0.049542 & $0.01566(3)$ & 0.015709 \\
\hline 0.5 & $0.04154(5)$ & 0.041506 & $0.015722(8)$ & 0.0157145 \\
\hline 1.0 & $0.03805(3)$ & 0.038051 & $0.015717(9)$ & 0.0157211 \\
\hline 5.0 & $0.030085(9)$ & 0.0300729 & $0.015776(4)$ & 0.0157739 \\
\hline 10.0 & $0.026682(9)$ & 0.0266732 & $0.015836(6)$ & 0.0158406 \\
\hline \hline
\end{tabular}

Table 10: $S^{221}$ compared with the expansions described in Appendix F for $m_{1}=m_{2}=m_{3}=100 \mathrm{GeV}$ and $m_{4}=m_{5}=0$. The sum over $n$ in Eq.(424) is restricted to $n \leq 20$. 


\section{References}

[1] A. Pais, Inward Bound Of Matter And Forces In The Physical World, Oxford, Uk: Clarendon (1986) New York, Usa: Oxford Univ. Pr. 1986).

[2] P. Mastrolia and E. Remiddi, way," Nucl. Phys. Proc. Suppl. 89 (2000) 76.

[3] K. G. Chetyrkin and A. Retey, Nucl. Phys. B 583 (2000) 3 [hep-ph/9910332.

[4] K. G. Chetyrkin and A. Retey, hep-ph/0007088.

[5] D. Bardin and G. Passarino, The standard model in the making: Precision study of the electroweak interactions, Oxford, UK: Clarendon (1999);

G. Montagna, F. Piccinini, O. Nicrosini, G. Passarino and R. Pittau, Comput. Phys. Commun. 76 (1993) 328;

G. Montagna, F. Piccinini, O. Nicrosini, G. Passarino and R. Pittau, Nucl. Phys. B 401 (1993) 3;

D. Y. Bardin, P. Christova, M. Jack, L. Kalinovskaya, A. Olchevski, S. Riemann and T. Riemann, Comput. Phys. Commun. 133 (2001) 229 arXiv:hep-ph/9908433.

[6] F. V. Tkachov, Nucl. Instrum. Meth. A 389 (1997) 309 hep-ph/9609429;

L. N. Bertstein, Functional Analysis and its Applications, 6(1972)66.

[7] G. Passarino, arXiv:hep-ph/0108252.

[8] A. Ghinculov and Y. P. Yao, arXiv:hep-ph/0106020;

M. Caffo, H. Czyz and E. Remiddi, Nucl. Phys. B 611 (2001) 503 arXiv:hep-ph/0103014;

S. Heinemeyer and G. Weiglein, arXiv:hep-ph/0102317;

A. Freitas, S. Heinemeyer, W. Hollik, W. Walter and G. Weiglein, arXiv:hep-ph/0101260;

T. Gehrmann and E. Remiddi, arXiv:hep-ph/0101147;

T. Gehrmann and E. Remiddi, Nucl. Phys. B 601 (2001) 287 arXiv:hep-ph/0101124;

A. Ghinculov and Y. P. Yao, Phys. Rev. D 63 (2001) 054510 arXiv:hep-ph/0006314;

T. Gehrmann and E. Remiddi, Nucl. Phys. Proc. Suppl. 89 (2000) 251 arXiv:hepph/0005232];

T. Gehrmann and E. Remiddi, Nucl. Phys. B 580 (2000) 485 arXiv:hep-ph/9912329;

L. Brucher, J. Franzkowski, A. Frink and D. Kreimer, Acta Phys. Polon. B 28 (1997) 835;

J. Fleischer, M. Y. Kalmykov and A. V. Kotikov, arXiv:hep-ph/9905379;

J. Fleischer, M. Y. Kalmykov and A. V. Kotikov, Phys. Lett. B 462 (1999) 169 arXiv:hepph/9905249];

J. Fleischer and O. L. Veretin, arXiv:hep-ph/9901402;

J. Fleischer, A. V. Kotikov and O. L. Veretin, Nucl. Phys. B 547 (1999) 343 arXiv:hepph/9808242];

M. Caffo, H. Czyz, S. Laporta and E. Remiddi, Nuovo Cim. A 111 (1998) 365 arXiv:hepth/9805118]; 
O. V. Tarasov, Nucl. Phys. B 502 (1997) 455 arXiv:hep-ph/9703319;

A. I. Davydychev, Acta Phys. Polon. B 28 (1997) 841 arXiv:hep-ph/9610510;

F. A. Berends, A. I. Davydychev and V. A. Smirnov, Nucl. Phys. Proc. Suppl. 51C (1996) 301 arXiv:hep-ph/9606244;

F. A. Berends, A. I. Davydychev and V. A. Smirnov, Nucl. Phys. B 478 (1996) 59 arXiv:hepph/9602396;

A. I. Davydychev and J. B. Tausk, Nucl. Phys. B 465 (1996) 507 arXiv:hep-ph/9511261;

J. Fujimoto, Y. Shimizu, K. Kato and T. Kaneko, Int. J. Mod. Phys. C 6 (1995) 525 [arXiv:hep-ph/9505270];

S. Bauberger and M. Bohm, Nucl. Phys. B 445 (1995) 25 arXiv:hep-ph/9501201;

N. I. Usyukina and A. I. Davydychev, Phys. Lett. B 348 (1995) 503 arXiv:hep-ph/9412356;

S. Bauberger, F. A. Berends, M. Bohm and M. Buza, Nucl. Phys. B 434 (1995) 383 arXiv:hepph/9409388];

J. Fleischer and O. V. Tarasov, Nucl. Phys. Proc. Suppl. 37B (1994) 115 arXiv:hepph/9407235; ;

S. Bauberger, M. Bohm, G. Weiglein, F. A. Berends and M. Buza, Nucl. Phys. Proc. Suppl. 37B (1994) 95 arXiv:hep-ph/9406404;

A. Ghinculov and J. J. van der Bij, Nucl. Phys. B 436 (1995) 30 arXiv:hep-ph/9405418.

[9] L. D. Landau, Nucl. Phys. 13 (1959) 181.

[10] D. Y. Bardin, L. V. Kalinovskaya and F. V. Tkachov, hep-ph/0012209.

[11] G. Passarino, hep-ph/0101299.

[12] A. Ghinculov and Y. Yao, Phys. Rev. D 63 (2001) 054510 hep-ph/0006314;

A. Ghinculov and Y. Yao, Mod. Phys. Lett. A 15 (2000) 1967 hep-ph/0004201;

A. Ghinculov and Y. Yao, Mod. Phys. Lett. A 15 (2000) 925 hep-ph/000221];

A. Ghinculov and Y. Yao, Nucl. Phys. B 516 (1998) 385 hep-ph/9702266.

[13] R. E. Cutkosky, J. Math. Phys. 1 (1960) 429;

G. 't Hooft and M. J. Veltman, In *Louvain 1973, Particle Interactions At Very High Energies, Part B*, New York 1973, 177-322 and CERN Geneva - CERN 73-9 (73,REC.OCT) $114 p$.

[14] G. Kallen, Quantum Electrodynamics Springer-verl. New York 1972.

[15] A. Ferroglia, G. Passarino and S. Uccirati, Numerical Evaluation of Feynman Diagrams: Two-Loop Vertices, work in preparation.

[16] R. Erdelyi et al., Higher Transcendental Functions vol. 1, Bateman Manuscript Project (McGraw-Hill 1953).

[17] T. Binoth and G. Heinrich, Nucl. Phys. B 585 (2000) 741 arXiv:hep-ph/0004013. 
[18] G. 't Hooft and M. Veltman, Nucl. Phys. B 44 (1972) 189.

[19] G. 't Hooft and M. Veltman, Nucl. Phys. B 153 (1979) 365.

[20] R. J. Eden, P. V. Landshoff, D. I. Olive, and J. C. Polkinghorne, The Analytic S Matrix, Cambridge Univ. Press, 1966.

[21] G. Passarino and M. Veltman, Nucl. Phys. B 160 (1979) 151.

[22] J. A. Vermaseren, math-ph/0010025.

[23] NAG Fortran Library, Mark 19, The Numerical Algorithms Group Ltd, Oxford UK. 1999.

[24] R. Erdelyi et al., Higher Transcendental Functions vol. 2, Bateman Manuscript Project (McGraw-Hill 1953).

[25] B. C. Carlson, J. Math. Phys. 44 (1965) 51.

[26] S. Bauberger, F. A. Berends, M. Bohm and M. Buza, Nucl. Phys. B 434 (1995) 383 arXiv:hepph/9409388.

[27] S. Bauberger and M. Bohm, Nucl. Phys. B 445 (1995) 25 arXiv:hep-ph/9501201.

[28] Z. Bern, L. Dixon and D. A. Kosower, Nucl. Phys. B 412 (1994) 751 hep-ph/9306240;

G. 't Hooft and M. Veltman, Nucl. Phys. B 153 (1979) 365.

[29] R. Barbieri, J. A. Mignaco and E. Remiddi, Nuovo Cim. A 11 (1972) 824.

[30] A. Devoto and D. W. Duke, Riv. Nuovo Cim. 7N6 (1984) 1.

[31] L. Lewin, Poly-logarithms and Associated Functions, North Holland (New York 1981). 